JOURNAL OF THE AMERICAN MATHEMATICAL SOCIETY

Volume 20, Number 2, April 2007, Pages 273-320

S 0894-0347(06)00544-3

Article electronically published on October 24, 2006

\title{
SUPERCUSPIDAL REPRESENTATIONS: AN EXHAUSTION THEOREM
}

JU-LEE KIM

\section{INTRODUCTION}

Let $k$ be a $p$-adic field of characteristic zero and residue characteristic $p$. Let $G$ be the group of $k$-points of a connected reductive group $\mathbf{G}$ defined over $k$. In 38, Yu gives a fairly general construction of supercuspidal representations of $G$ in a certain tame situation. In this paper, subject to some hypotheses on $\mathbf{G}$ and $k$, we prove that all supercuspidal representations arise through his construction.

While there have been numerous constructions of supercuspidal representations, the question of whether they are exhaustive is resolved only for depth zero representations [31, 28, and for groups of type $A_{n}$ such as $\mathbf{G L}_{n}$ [5, 22, 29, $\mathbf{S L}_{n}, 6$, 7]. In 29], Moy proves the exhaustiveness for Howe's construction of supercuspidal representations via the generalized Jacquet-Langlands correspondence in the tame case. In 22, Howe and Moy prove it by analyzing Hecke algebras when $p>n$. In 5], Bushnell and Kutzko construct supercuspidal representations and prove their exhaustiveness by analyzing simple types and split types with no assumption on $k$. Recently, Stevens showed that any supercuspidal representation of classical groups of positive depth contains a certain semisimple character 34. However, since no analogue of the Jacquet-Langlands correspondence for general groups has been developed yet, and since types, or Hecke algebras for general groups, are far less understood than for $\mathbf{G L}_{n}$, it is not easy to extend their methods to other groups.

In this paper, we approach this problem via harmonic analysis on $G$. We now briefly describe the main idea of the proof. From now on, we assume that the residue characteristic $p$ of $k$ is sufficiently large (see 33.4 for the precise condition). We first prove that any supercuspidal representation is either of depth zero or otherwise contains a K-type constructed in 27. This we do by relating the Plancherel formulas on $G$ and on its Lie algebra $\mathfrak{g}$ and by using some results on asymptotic expansions [27]. We relate the K-type further to a supercuspidal type constructed in 38 by analyzing appropriate Hecke algebras and Jacquet modules. Before expanding our account of the main strategy of the proof, we first recall some results on $\Gamma$-asymptotic expansions.

0.1. Results on $\Gamma$-asymptotic expansions. Let $\mathcal{E}:=\mathcal{E}(G)$ denote the set of all equivalence classes of irreducible admissible representations of $G$. We use the same notation for a representation $\pi$ and its equivalence class. For $\left(\pi, V_{\pi}\right) \in \mathcal{E}$, let $\Theta_{\pi}$ be the character of $\pi$. Let $\mathcal{B}(\mathbf{G}, k)$ be the extended building of $\mathbf{G}$ over $k$. In

Received by the editors February 20, 2004.

2000 Mathematics Subject Classification. Primary 22E50; Secondary 22E35, 20 G25.

Key words and phrases. Supercuspidal representations, K-types, $p$-adic groups.

(C)2006 American Mathematical Society Reverts to public domain 28 years from publication 
[27, we found a certain character expansion of $\Theta_{\pi}$ depending on K-types contained in $\left(\pi, V_{\pi}\right)$. The construction of K-types is based on a (strongly) good positive $G$-datum $\Sigma$ which is a quadruple (see Definitions 5.1 and 5.3 ). However, if $\Sigma$ is strongly good, $\Sigma$ can be alternatively described as a pair $\Sigma=(\Gamma, y)$ of a semisimple element $\Gamma \in \mathfrak{g}$ and $y \in \mathcal{B}\left(\mathbf{C}_{\mathbf{G}}(\Gamma), k\right)$ satisfying the following (see Definitions 5.1 and 5.3 and Remark (5.10):

(i) $\Gamma=\Gamma_{d}+\Gamma_{d-1}+\cdots+\Gamma_{0}$ where $\Gamma_{i}, 0 \leq i \leq d-1$, is a $G$-good element of depth $-r_{i}$ and $\Gamma_{d}$ is either zero or an element in the center of $\mathfrak{g}$ of depth $-r_{d}$. Set $r_{d}:=r_{d-1}$ if $\Gamma_{d}=0$.

(ii) $0<r_{0}<r_{1}<\cdots<r_{d-1}$. If $\Gamma_{d} \neq 0, r_{d-1}<r_{d}$.

(iii) $\mathbf{C}_{\mathbf{G}}(\Gamma)=\mathbf{G}^{0} \subsetneq \mathbf{G}^{1} \subsetneq \cdots \subsetneq \mathbf{G}^{d-1} \subsetneq \mathbf{G}$ where $\mathbf{G}^{i}=\mathbf{C}_{\mathbf{G}}\left(\Gamma_{d}+\Gamma_{d-1}+\cdots \Gamma_{i}\right)$. Set $s_{i}:=\frac{r_{i}}{2}$ and $\vec{s}^{+}:=\left(0^{+}, s_{0}^{+}, s_{1}^{+}, \cdots, s_{d-1}^{+}\right)$. Then the associated K-type (which we denote by $\left(K_{\Sigma}^{+}, \phi_{\Sigma}\right)$ in the text) is $\left(\vec{G}_{y, \vec{s}^{+}}, \chi_{\Gamma}\right)$, where $\vec{G}_{y, \vec{s}^{+}}$is an open compact subgroup defined in [38] and $\chi_{\Gamma}$ is the character on $\vec{G}_{y, \vec{s}^{+}}$represented by $\Gamma$ via a logarithmic map. Any irreducible admissible representation containing $\chi_{\Gamma}$ when restricted to $\vec{G}_{y, \vec{s}^{+}}$has depth $\varrho=r_{d}>0$. Moreover, such a representation is not supercuspidal in general; that is, $\left(\vec{G}_{y, \vec{s}^{+}}, \chi_{\Gamma}\right)$ is not necessarily a supercuspidal type.

The main result of 27] states that if $\pi$ contains $\left(\vec{G}_{y, \vec{s}^{+}}, \chi_{\Gamma}\right)$, for any $f \in$ $C_{c}^{\infty}\left(\mathfrak{g}_{s_{d-1}^{+}}\right)$, then $\Theta_{\pi}(f \circ \log )=\sum_{\mathcal{O} \in \mathcal{O}(\Gamma)} c_{\mathcal{O}}(\pi) \widehat{\mu_{\mathcal{O}}}(f)$. Here, $\mathfrak{g}_{r^{+}}=\bigcup_{x \in \mathcal{B}(\mathbf{G}, k)} \mathfrak{g}_{x, r^{+}}$, $r \in \mathbb{R}, \mathcal{O}(\Gamma)$ is the set of $G$-orbits whose closures contain $\Gamma$, and $\widehat{\mu_{\mathcal{O}}}$ is the Fourier transform of the orbital integral $\mu_{\mathcal{O}}$. In [27, we also define a certain subspace $\delta^{\Gamma}$ of the space of $G$-invariant distributions on $\mathfrak{g}$ having the property that $\jmath^{\Gamma}$, when restricted to the image of the Fourier transform of $C_{c}^{\infty}\left(\mathfrak{g}_{s_{d-1}^{+}}\right)$, coincides with the finite-dimensional space spanned by $\mu_{\mathcal{O}}, \mathcal{O} \in \mathcal{O}(\Gamma)$. An important property of this expansion is that there are test functions $f_{\mathcal{O}}$ in $C_{c}^{\infty}(\mathfrak{g})$ indexed by $\mathcal{O} \in \mathcal{O}(\Gamma)$ such that, for two $G$-invariant distributions $\mathrm{T}_{1}$ and $\mathrm{T}_{2}$ on $\mathfrak{g}$ with their Fourier transforms $\widehat{\mathrm{T}}_{1}, \widehat{\mathrm{T}}_{2}$ in $g^{\Gamma}$, if $\widehat{\mathrm{T}}_{1}\left(f_{\mathcal{O}}\right)=\widehat{\mathrm{T}}_{2}\left(f_{\mathcal{O}}\right)$ for all $\mathcal{O} \in \mathcal{O}(\Gamma)$, then $\mathrm{T}_{1} \equiv \mathrm{T}_{2}$ on $C_{c}^{\infty}\left(\mathfrak{g}_{s_{d-1}^{+}}\right)$ (see Theorem 9.6).

0.2. First step. Let $\mathcal{E}^{t}$ be the set of equivalence classes of irreducible tempered representations. We first show that almost every irreducible tempered representation $\left(\pi, V_{\pi}\right)$ is either of depth zero or otherwise contains $\left(\vec{G}_{y, \vec{s}^{+}}, \chi_{\Gamma}\right)$ for some $(\Gamma, y)$.

We begin by observing that, thanks to the Plancherel formulas on $\mathfrak{g}$ and $G$ ([13]), we have the equality

$$
\int_{\mathfrak{g}} \widehat{f}(X) d X=f(0)=\int_{\mathcal{E}^{t}} \Theta_{\pi}(f \circ \log ) d \pi
$$

for $f \in C_{c}^{\infty}(\mathfrak{g})$ supported in a small neighborhood of 0 . Here, note that we have identified $\mathfrak{g}$ with the unitary dual $\widehat{\mathfrak{g}}$ of $\mathfrak{g}$. Now, refining this equality, we will find a match between spectral decomposition factors of each side of (0.1), parameterized by some equivalence classes on the union of $\{0\}$ and semisimple elements satisfying (i) $-($ iii $)$.

Let $\Gamma$ be a semisimple element as above or let $\Gamma=0$. Then we define $\mathfrak{g}_{\Gamma}:={ }^{G}(\Gamma+$ $\mathfrak{g}_{0}^{0}$ ) where $\mathfrak{g}^{0}$ is the Lie algebra of $G^{0}=C_{G}(\Gamma)$ (recall that $\mathfrak{g}_{0}^{0}=\bigcup_{x \in \mathcal{B}\left(\mathbf{G}^{0}, k\right)} \mathfrak{g}_{x, 0}^{0}$ ). Each $\mathfrak{g}_{\Gamma}$ is a $G$-domain, an open and closed $G$-invariant subset of $\mathfrak{g}$. We say that two such semisimple elements $\Gamma$ and $\Gamma^{\prime}$ are equivalent if $\mathfrak{g}_{\Gamma}=\mathfrak{g}_{\Gamma^{\prime}}$ (see Definition 7.4). Let $\mathfrak{S}$ be the set of equivalence classes of the $\Gamma$ 's. Then, $\mathfrak{g}$ is the disjoint union 
of $G$-domains $\mathfrak{g}_{\Gamma}, \Gamma \in \mathfrak{S}$ (see Proposition [7.6), and we have

$$
\int_{\mathfrak{g}} \widehat{f}(X) d X=\sum_{\Gamma \in \mathfrak{S}} \int_{\mathfrak{g}_{\Gamma}} \widehat{f}(X) d X
$$

On the other hand, each $\Gamma \in \mathfrak{S}$ also parameterizes a subset $\mathcal{E}_{\Gamma}$ of $\mathcal{E}$. Roughly speaking, $\mathcal{E}_{\Gamma}$ is the subset of $\mathcal{E}$ which consists of $\left(\pi, V_{\pi}\right) \in \mathcal{E}$ containing $\left(\vec{G}_{y, \vec{s}^{+}}, \chi_{\Gamma^{\prime}}\right)$ for some $\Gamma^{\prime} \sim \Gamma$ and $y \in \mathcal{B}\left(\mathbf{C}_{\mathbf{G}}\left(\Gamma^{\prime}\right), k\right)$, and $\mathcal{E}_{0}$ is the set of depth zero representations (see Definition 8.5 and Remark 11.3 for details). Moreover, $\mathcal{E}_{\Gamma}=\mathcal{E}_{\Gamma^{\prime}}$ if $\Gamma$ and $\Gamma^{\prime}$ are equivalent, and $\varepsilon_{\Gamma} \cap \varepsilon_{\Gamma^{\prime}}=\emptyset$ otherwise (see Lemma 8.6). Setting $\mathcal{E}_{\mathfrak{S}}^{t}:=\left(\bigcup_{\Gamma \in \mathfrak{S}}^{0} \mathcal{E}_{\Gamma}^{t}\right)$ where $\mathcal{E}_{\Gamma}^{t}=\mathcal{E}^{t} \cap \mathcal{E}_{\Gamma}$, we have

$$
\int_{\mathcal{E}^{t}} \Theta_{\pi}(f \circ \log ) d \pi=\sum_{\Gamma \in \mathfrak{S}} \int_{\mathcal{E}_{\Gamma}^{t}} \Theta_{\pi}(f \circ \log ) d \pi+\int_{\mathcal{E}^{t} \backslash \mathcal{E}_{\mathfrak{S}}^{t}} \Theta_{\pi}(f \circ \log ) d \pi .
$$

It is obvious that $\mathcal{E}_{\mathfrak{S}}^{t} \subset \mathcal{E}^{t}$. Our claim above is that $\mathcal{E}^{t} \backslash \mathcal{E}_{\mathfrak{S}}^{t}$ has Plancherel measure zero.

Now, we match terms parameterized by $\Gamma \in \mathfrak{S}$ (see \$10):

$$
\int_{\mathfrak{g}_{\Gamma}} \widehat{f}(X) d X=\int_{\mathcal{E}_{\Gamma}^{t}} \Theta_{\pi}(f \circ \log ) d \pi
$$

for $f \in C_{c}^{\infty}\left(\mathfrak{g}_{0^{+}}\right)$if $\Gamma \sim 0$ and for $f \in C_{c}^{\infty}\left(\mathfrak{g}_{s_{d-1}^{+}}\right)$if $\Gamma \not 0$. A similar equality was considered in [19] when $\Gamma$ is regular and in [25] when $\Gamma$ is a good element. If $\Gamma \sim 0$, this is already proven in 25]. If $\Gamma \not 0$, we regard both sides of (0.2) as distributions on $C_{c}^{\infty}\left(\mathfrak{g}_{s_{d-1}^{+}}\right)$, and we denote the distributions on the left and the right side of (0.2) by $\mathrm{T}_{\ell}$ and $\mathrm{T}_{r}$, respectively. We first need to prove that the $\mathcal{J}^{-\Gamma}$ from [27] (see also the previous subsection) contain both distributions $\widehat{\mathrm{T}}_{\ell}$ and $\widehat{\mathrm{T}}_{r}$. Then, by matching $\widehat{\mathrm{T}}_{\ell}\left(f_{\mathcal{O}}\right)=\widehat{\mathrm{T}}_{r}\left(f_{\mathcal{O}}\right)$ for each test function $f_{\mathcal{O}}, \mathcal{O} \in \mathcal{O}(-\Gamma)$, found in [27, we verify that they are equal on $C_{c}^{\infty}\left(\mathfrak{g}_{s_{d-1}}^{+}\right)$. Since we prove this equality by matching them only on test functions, and since these test functions have the property that $\left\{\pi \in \mathcal{E}^{t} \mid \Theta_{\pi}\left(\widehat{f}_{\mathcal{O}}\right) \neq 0\right.$, for some $\left.\mathcal{O} \in \mathcal{O}(-\Gamma)\right\}$ is a subset of $\mathcal{E}_{\Gamma}^{t}$, we do not need any explicit knowledge of the Plancherel measure $d \pi$.

Using this, we can also prove that the equality in (0.2) holds for any characteristic function $f_{x, s}$ of a lattice $\mathfrak{g}_{x, s}$ with $x \in \mathcal{B}(\mathbf{G}, k)$ and $s>0$. Summing over all $\Gamma \in \mathfrak{S}$, we have

$$
\begin{aligned}
\int_{\mathfrak{g}} \widehat{f}_{x, s}(X) d X & =\sum_{\Gamma \in \mathfrak{S}} \int_{\mathfrak{g}_{\Gamma}} \widehat{f}_{x, s}(X) d X \\
& =\sum_{\Gamma \in \mathfrak{S}} \int_{\mathcal{E}_{\Gamma}^{t}} \Theta_{\pi}\left(f_{x, s} \circ \log \right) d \pi=\int_{\mathcal{E}^{t}} \Theta_{\pi}\left(f_{x, s} \circ \log \right) d \pi,
\end{aligned}
$$

which will lead to a proof that almost every irreducible tempered representation (in particular, supercuspidal representation) is an element of $\varepsilon_{\Gamma}^{t}$ for some $\Gamma \in \mathfrak{S}$ (Theorems 11.1 and 11.4).

0.3. Generic $G$-datum. The construction in 38 is based on a generic $G$-datum which consists of a quintuple $\Sigma_{Y}=(\overrightarrow{\mathbf{G}}, y, \vec{r}, \vec{\phi}, \rho)$ satisfying the following five conditions (see [38, $\S 3]$ or $\S 12$ ):

D1. $\overrightarrow{\mathbf{G}}=\left(\mathbf{G}^{0}, \mathbf{G}^{1}, \cdots, \mathbf{G}^{d}=\mathbf{G}\right)$ is a tamely ramified Levi sequence and $\mathbf{Z}_{\mathbf{G}^{0}} / \mathbf{Z}_{\mathbf{G}}$ is $k$-anisotropic, where $\mathbf{Z}_{\mathbf{G}^{0}}$ (resp. $\mathbf{Z}_{\mathbf{G}}$ ) is the center of $\mathbf{G}^{0}$ (resp. $\mathbf{G}$ ). 
D2. $y \in \mathcal{B}\left(\mathbf{G}^{0}, k\right)$.

D3. $\vec{r}=\left(r_{0}, r_{1}, \cdots, r_{d-1}, r_{d}\right)$ is a sequence of positive real numbers with $0<$ $r_{0}<\cdots<r_{d-2}<r_{d-1} \leq r_{d}$ if $d>0,0 \leq r_{0}$ if $d=0$.

D4. $\vec{\phi}=\left(\phi_{0}, \phi_{1}, \cdots, \phi_{d}\right)$ is a sequence of quasi-characters; $\phi_{i}, 0 \leq i \leq d-1$, is a $G^{i+1}$-generic character of $G^{i}$ of depth $r_{i}$ at $y$. If $r_{d-1}<r_{d}, \phi_{d}$ is a generic character of $G^{d}$ of depth $r_{d}$ at $y$, and $\phi_{d}$ is trivial otherwise.

D5. $\rho$ is an irreducible representation of $G_{[y]}^{0}$, the stabilizer in $G^{0}$ of the image $[y]$ of $y$ in the reduced building of $\mathbf{G}^{0}$, such that $\rho \mid G_{y, 0^{+}}^{0}$ is a multiple of the trivial representation and $c-\operatorname{Ind}_{G_{[y]}^{0}}^{G^{0}}(\rho)$ is irreducible and supercuspidal.

Based on the above data, Yu constructs a pair $\left(K_{\Sigma_{Y}}, \rho_{\Sigma_{Y}}\right)$ of an open compact modulo center subgroup $K_{\Sigma_{Y}}$ and its irreducible representation $\rho_{\Sigma_{Y}}$ such that $c$-Ind $K_{\Sigma_{Y}}^{G} \rho_{\Sigma_{Y}}$ is supercuspidal. Denote the resulting supercuspidal representation $c$-Ind $K_{\Sigma_{Y}}^{G} \rho_{\Sigma_{Y}}$ by $\pi_{\Sigma_{Y}}$.

0.4. Second step. Now, let $\left(\pi, V_{\pi}\right)$ be a supercuspidal representation of $G$. We want to prove that there is a generic $G$-datum $\Sigma_{Y}$ such that $\pi \simeq \pi_{\Sigma_{Y}}$. Since the case of depth zero supercuspidal representations is already known (see [28, 31, (6.6), (6.8)]), we may assume that $\pi$ is of positive depth. Then from the first step, $\pi$ contains a K-type $\left(\vec{G}_{y, \vec{s}^{+}}, \chi_{\Gamma}\right)$ for some $(\Gamma, y)$. Let $\Gamma_{i}, \mathbf{G}^{i}$ and $r_{i}$ be as in $(i)-(i i i)$. Let $\phi_{i}$ be a quasi-character of $G^{i}$ extending the character $\chi_{\Gamma_{i}}$ of $G_{y, s_{i}^{+}}^{i}$ defined by $\Gamma_{i}$. Under our hypothesis, $\chi_{\Gamma_{i}}$ always extends to a quasi-character of $G^{i}$ (see Lemma 5.5). Let $\rho$ be an irreducible component of the $G_{[y]}^{0}$-representation $\phi^{-1} \otimes\left(\pi \mid V_{\pi}^{\chi_{\Gamma}}\right)$ where $\phi=\prod_{i}\left(\phi_{i} \mid G_{[y]}^{0}\right)$ and $V_{\pi}^{\chi_{\Gamma}}$ is the $\chi_{\Gamma}$ isotypic component in $V_{\pi}$. To show that $(\overrightarrow{\mathbf{G}}, y, \vec{r}, \vec{\phi}, \rho)$ satisfies the desired properties D1-D5, we need to verify that

(a) $\mathbf{Z}_{\mathbf{G}^{0}} / \mathbf{Z}_{\mathbf{G}}$ is anisotropic,

(b) $c$-Ind $G_{[y]}^{G^{0}}(\rho)$ is irreducible and supercuspidal.

We prove (a) by analyzing appropriate Hecke algebras $(\S 13-\S 14)$ and (b) by analyzing appropriate Jacquet modules $(\S 15-\S 17)$.

Let $\Sigma_{Y}$ be the generic $G$-datum $(\vec{G}, y, \vec{r}, \vec{\phi}, \rho)$ associated to $\left(\pi, V_{\pi}\right)$ found as above. Lastly, we show $\chi_{\Gamma}$ on $\vec{G}_{y, \vec{s}^{+}}$can be extended further to $\rho_{\Sigma_{Y}}$ on $K_{\Sigma_{Y}}$ $(\S 18)$ and $\left(\pi, V_{\pi}\right)$ still contains $\left(K_{\Sigma_{Y}}, \rho_{\Sigma_{Y}}\right)$. Then, by Frobenius reciprocity, we can conclude that $\pi$ is in fact the supercuspidal representation constructed from $\Sigma_{Y}$, that is, $\pi \simeq \pi_{\Sigma_{Y}}$.

Generally speaking, different generic $G$-datums can yield isomorphic supercuspidal representations (this is the case e.g. for $G$-conjugate generic $G$-datums). The question of when exactly the resulting supercuspidal representations are isomorphic is settled by recent work of Hakim and Murnaghan [14]; we will not discuss it here.

If $G$ is one of the classical groups considered in 24, the corresponding datum in 24 is $\left(\Gamma, G_{[y]}^{0}, \rho\right)$. The result of this paper also implies that the K-types constructed in 24] form a complete set for the classical groups considered in that paper.

In the first three sections, we review some facts about Moy-Prasad filtrations and relevant results on buildings. Otherwise, reviews of many necessary results (in particular from [27]) are spread throughout this paper before they are used. The first step is carried out in $\S 4-\S 11$. We review Yu's construction of supercuspidal representations in $\S 12$. The second step is done in $\S 13-\S 17$. In $\S 18$, we compare 
$\left(\vec{G}_{y, s^{+}}, \chi_{\Gamma}\right)$ and $\left(K_{\Sigma_{Y}}, \rho_{\Sigma_{Y}}\right)$. Finally in $\S 19$, we conclude that all supercuspidal representations arise through Yu's construction (Theorem 19.1).

Most notation is used throughout the paper once it is defined. The table of some selected notation is available at the end of this paper.

\section{NotATION AND CONVENTIONS}

Let $k$ be a $p$-adic field (a finite extension of $\mathbb{Q}_{p}$ ) with residue field $\mathbb{F}_{p^{n}}$. Let $\nu=\nu_{k}$ be the valuation on $k$ such that $\nu\left(k^{\times}\right)=\mathbb{Z}$. Let $\bar{k}$ be an algebraic closure of $k$. For an extension field $E$ of $k$, let $\nu_{E}$ be the valuation on $E$ extending $\nu$. We will just write $\nu$ for $\nu_{E}$. Let $\mathcal{O}_{E}$ be the ring of integers of $E$ with prime ideal $\mathfrak{p}_{E}$.

Let $\Lambda$ be a fixed additive character of $k$ such that $\Lambda \mid \mathcal{O}_{k} \neq 1$ and $\Lambda \mid \mathfrak{p}_{k}=1$.

Let $\mathbf{G}$ be a connected reductive group defined over $k$, and let $\mathfrak{g}$ be the Lie algebra of $\mathbf{G}$. Denote the group of $E$-rational points of $\mathbf{G}$ by $\mathbf{G}(E)$ and the Lie algebra of $E$-rational points of $\mathfrak{g}$ by $\mathfrak{g}(E)$. We denote $\mathbf{G}(k)$ and $\mathfrak{g}(k)$ by $G$ and $\mathfrak{g}$, respectively. Similarly, the linear duals of $\mathfrak{g}$ and $\mathfrak{g}(E)$ are denoted by $\mathfrak{g}^{*}$ and $\mathfrak{g}^{*}(E)$, respectively. We write $\mathfrak{g}^{*}$ for $\mathfrak{g}^{*}(k)$. Let $\mathbf{Z}_{\mathbf{G}}$ denote the center of $\mathbf{G}$ and $\mathfrak{z}_{\mathfrak{g}}$ the Lie algebra of $\mathbf{Z}_{\mathbf{G}}$. Let $\mathbf{G}^{\text {der }}$ denote the derived group of $\mathbf{G}$ and $\mathfrak{g}^{\text {der }}$ the Lie algebra of $\mathbf{G}^{\text {der }}$. In general, we use bold characters $\mathbf{H}, \mathbf{M}, \mathbf{N}$, etc., to denote algebraic groups and $\mathfrak{h}, \mathfrak{m}, \mathfrak{n}$ to denote their Lie algebras. If they are defined over $k$, we will use the corresponding Roman characters $H, M$ and $N$ to denote the groups of $k$-points and $\mathfrak{h}, \mathfrak{m}$ and $\mathfrak{n}$ to denote the Lie algebras of $H, M$ and $N$.

Let $\mathcal{N}$ denote the set of nilpotent elements in $\mathfrak{g}$. There are different notions of nilpotency. However, since we assume that $\operatorname{char}(k)=0$, those notions are all the same. We refer to [12, 30] for more discussion of this.

If $X$ is a topological space with a Borel measure $d x$ and if $Y$ is a Borel subset of $X, \operatorname{vol}_{X}(Y)$ denotes the volume of $Y$ with respect to $d x$.

For any given set $W$, let $|W|$ denote the cardinality of $W$.

For any subset $S$ in $\mathfrak{g}$ or in $G$, we denote by $[S]$ the characteristic function on $S$ and by $-S$ the set $\{-s \mid s \in S\}$. For $g \in G,{ }^{g} Z$ denotes $g Z g^{-1}$.

Let $\tilde{\mathbb{R}}:=\mathbb{R} \cup\left\{r^{+} \mid r \in \mathbb{R}\right\}$. We define an ordering on $\tilde{\mathbb{R}}$ extending the one on $\mathbb{R}$ : let $r, s \in \mathbb{R}$. Then, $r<r^{+}$. If $r<s$, then $r<s^{+}, r^{+}<s^{+}$and $r^{+}<s$. We define an addition on $\tilde{\mathbb{R}}$ extending the one on $\mathbb{R}$ : for $r, s \in \mathbb{R}, r^{+}+s=(r+s)^{+}=r^{+}+s^{+}$. Set $\left(r^{+}\right)^{+}:=r^{+}$.

Finally, we will not distinguish between representations and their isomorphism classes.

\section{Moy-Prasad FiLtrations}

1.1. Apartments and buildings. For a finite extension $E$ of $k$, let $\mathcal{B}(\mathbf{G}, E)$ denote the extended Bruhat-Tits building of $\mathbf{G}$ over $E$. Recall that $\mathcal{B}(\mathbf{G}, E)=$ $\mathcal{B}(\mathbf{D G}, E) \times\left(\mathbf{X}_{*}\left(\mathbf{Z}_{\mathbf{G}}, E\right) \otimes \mathbb{R}\right)$, where $\mathbf{D G}$ is the derived group of $\mathbf{G}$ and $\mathbf{X}_{*}\left(\mathbf{Z}_{\mathbf{G}}, E\right)$ is the abelian group of $E$-rational cocharacters of the center $\mathbf{Z}_{\mathbf{G}}$ of $\mathbf{G}$. For a maximal $E$-split torus $\mathbf{T}$ in $\mathbf{G}$, let $\mathcal{A}(\mathbf{T}, E)$ be the corresponding apartment over $E$. It is known that for any tamely ramified finite Galois extension $E^{\prime}$ of $E, \mathcal{B}(\mathbf{G}, E)$ can be embedded into $\mathcal{B}\left(\mathbf{G}, E^{\prime}\right)$ and its image is equal to the set of the Galois fixed points in $\mathcal{B}\left(\mathbf{G}, E^{\prime}\right)$ (see [33, (5.11)] or [32]).

1.2. Moy-Prasad filtrations. Let $(x, r) \in \mathcal{B}(\mathbf{G}, E) \times \mathbb{R}$. Regarding $\mathbf{G}$ as a group defined over $E$, Moy and Prasad define $\mathfrak{g}(E)_{x, r}$ and also $\mathbf{G}(E)_{x, r}$ if $r \geq 0$ 
with respect to the valuation normalized as follows (31]): let $E^{u}$ be the maximal unramified extension of $E$, and let $L$ be the minimal extension of $E^{u}$ over which $\mathbf{G}$ splits. Then the valuation used by Moy and Prasad maps $L^{\times}$onto $\mathbb{Z}$.

In a similar way, with respect to our normalized valuation $\nu$, we can define filtrations in $\mathfrak{g}(E)$ and $\mathbf{G}(E)$. Then our $\mathfrak{g}(E)_{x, r}$ and $\mathbf{G}(E)_{x, r}$ correspond to their $\mathfrak{g}(E)_{x, e l r}$ and $\mathbf{G}(E)_{x, e l r}$, respectively, where $e=e(E / k)$ is the ramification index of $E$ over $k$ and $\ell=\left[L: E^{u}\right]$. Hence, if $\varpi_{E}$ is a uniformizing element of $E$, our filtrations satisfy $\varpi_{E} \mathfrak{g}(E)_{x, r}=\mathfrak{g}(E)_{x, r+\frac{1}{e}}$ while theirs satisfy $\varpi_{E} \mathfrak{g}(E)_{x, r}=$ $\mathfrak{g}(E)_{x, r+\ell}$.

This normalization is chosen to have the following property (see also [1, (1.4.1)]): for a tamely ramified Galois extension $E^{\prime}$ of $E$ and $x \in \mathcal{B}(\mathbf{G}, E) \subset \mathcal{B}\left(\mathbf{G}, E^{\prime}\right)$, we have

$$
\mathfrak{g}(E)_{x, r}=\mathfrak{g}\left(E^{\prime}\right)_{x, r} \cap \mathfrak{g}(E)
$$

If $r>0$, we also have

$$
\mathbf{G}(E)_{x, r}=\mathbf{G}\left(E^{\prime}\right)_{x, r} \cap \mathbf{G}(E) .
$$

For simplicity, we put $\mathfrak{g}_{x, r}:=\mathfrak{g}(k)_{x, r}$, etc., and $\mathcal{B}(G):=\mathcal{B}(\mathbf{G}, k)$. We will also use the following notation. Let $r \in \mathbb{R}$.

(1) $\mathfrak{g}_{x, r^{+}}=\bigcup_{s>r} \mathfrak{g}_{x, s}$ and $G_{x,|r|^{+}}=\bigcup_{s>|r|} G_{x, s}, \quad x \in \mathcal{B}(G)$.

(2) $\mathfrak{g}_{x, r}^{*}=\left\{\chi \in \mathfrak{g}^{*} \mid \chi\left(\mathfrak{g}_{x,(-r)^{+}}\right) \subset \mathfrak{p}_{k}\right\}, \quad x \in \mathcal{B}(G)$.

(3) $\mathfrak{g}_{r}=\bigcup_{x \in \mathcal{B}(G)} \mathfrak{g}_{x, r}$ and $\mathfrak{g}_{r^{+}}=\bigcup_{s>r} \mathfrak{g}_{s}$.

(4) $G_{r}=\bigcup_{x \in \mathcal{B}(G)} G_{x, r}$ and $G_{r^{+}}=\bigcup_{s>r} G_{s}$ for $r \geq 0$.

The hypothesis $(\mathrm{HB})$ in 3.4 is concerned with identifying $\mathfrak{g}_{x, r}^{*}$ with $\mathfrak{g}_{x, r}$ via an appropriate bilinear form B on $\mathfrak{g}$ (see [4, (4.1)]).

1.3. Root decomposition. Let $\mathbf{T}$ be a maximal $k$-torus in $\mathbf{G}$ and $E$ a finite extension of $k$ over which $\mathbf{T}$ splits. Let $\Phi(\mathbf{G}, \mathbf{T}, E)$ be the set of $E$-roots of $\mathbf{T}$ in $\mathbf{G}$, and let $\Psi(\mathbf{G}, \mathbf{T}, E)$ be the corresponding set of affine roots in $\mathbf{G}$. If $\psi \in \Psi(\mathbf{G}, \mathbf{T}, E)$, let $\dot{\psi} \in \Phi(\mathbf{G}, \mathbf{T}, E)$ be the gradient of $\psi$, and let $\mathfrak{g}(E)_{\dot{\psi}} \subset \mathfrak{g}(E)$ be the root space corresponding to $\dot{\psi}$. We denote the open compact abelian group in $\mathfrak{g}(E)_{\dot{\psi}}$ corresponding to $\psi$ by $\mathfrak{g}(E)_{\psi}([30,(3.2)])$.

Let $\mathbf{X}_{*}(\mathbf{T}, E)$ be the set of cocharacters of $\mathbf{T}$, and let $\mathbf{X}^{*}(\mathbf{T}, E)$ be the set of characters of $\mathbf{T}$. For $r \in \tilde{\mathbb{R}}$, let

$$
\mathfrak{t}(E)_{r}=\left\{\Gamma \in \mathfrak{t}(E) \mid \nu(d \chi(\Gamma)) \geq r \text { for all } \chi \in \mathbf{X}^{*}(\mathbf{T}, E)\right\} .
$$

Then, for $x \in \mathcal{A}(\mathbf{G}, \mathbf{T}, E)$, we have

$$
\mathfrak{g}(E)_{x, r}=\mathfrak{t}(E)_{r}+\sum_{\psi \in \Psi(\mathbf{G}, \mathbf{T}, E), \psi(x) \geq r} \mathfrak{g}(E)_{\psi} .
$$

Let $\mathbf{T}$ be a maximal $k$-torus in $\mathbf{G}$ which splits over a tamely ramified finite Galois extension $E$ of $k$. Then, we write $\mathcal{A}(\mathbf{G}, \mathbf{T}, k)$ for $\mathcal{A}(\mathbf{G}, \mathbf{T}, E) \cap \mathcal{B}(\mathbf{G}, k)$. This definition is independent of the choice of $E$ [38]. Moreover, $\mathcal{A}(\mathbf{G}, \mathbf{T}, k)$ is the set of Galois fixed points in $\mathcal{A}(\mathbf{G}, \mathbf{T}, E)$.

\section{Twisted Levi Sequences}

Definition 2.1 (38]). Let $\mathbf{G}$ be a connected reductive $k$-group. Let $\overrightarrow{\mathbf{G}}:=\left(\mathbf{G}^{0}, \cdots\right.$, $\mathbf{G}^{d}=\mathbf{G}$ ) be a sequence of connected reductive $k$-groups with $\mathbf{G}^{0} \subsetneq \mathbf{G}^{1} \subsetneq \cdots \subsetneq \mathbf{G}^{d}$. 
(1) If each $\mathbf{G}^{i}$ is a $k$-split Levi subgroup of $\mathbf{G}, \overrightarrow{\mathbf{G}}$ is called a $k$-Levi sequence in G.

(2) If there exists a (tamely ramified) finite extension $E / k$ such that $\mathbf{G}^{0} \otimes E$ is split and if $\overrightarrow{\mathbf{G}} \otimes E=\left(\mathbf{G}^{0} \otimes E, \cdots, \mathbf{G}^{d} \otimes E\right)$ is an $E$-Levi sequence in $\mathbf{G}^{d} \otimes E$, then $\overrightarrow{\mathbf{G}}$ is called a (tamely ramified) twisted Levi sequence in $\mathbf{G}$ and $E$ is called a splitting field of $\overrightarrow{\mathbf{G}}$.

Note that any subsequence of a tamely ramified twisted Levi sequence $\overrightarrow{\mathbf{G}}$ is also a tamely ramified twisted Levi sequence.

2.2. Let $(\mathbf{H}, \mathbf{G})$ be a tamely ramified twisted Levi sequence and $E$ a tamely ramified Galois extension $E$ of $k$ over which $(\mathbf{H}, \mathbf{G})$ splits. Since $\mathbf{H}(E)$ is a Levi subgroup of $\mathbf{G}(E)$, there is a Galois equivariant embedding of $\mathcal{B}(\mathbf{H}, E)$ into $\mathcal{B}(\mathbf{G}, E)$, which in turn induces an embedding of $\mathcal{B}(\mathbf{H}, k)$ into $\mathcal{B}(\mathbf{G}, k)$ (see [1, $\S 1.9$ ] or [38, (2.11)]). Such embeddings are unique modulo translation by $\mathbf{X}_{*}\left(\mathbf{Z}_{\mathbf{H}}, k\right) \otimes \mathbb{R}$. However, the images remain the same.

Fix an embedding $i: \mathcal{B}(\mathbf{H}, k) \longrightarrow \mathcal{B}(\mathbf{G}, k)$. Then we will regard $\mathcal{B}(\mathbf{H}, k)$ as a subset of $\mathcal{B}(\mathbf{G}, k)$ and write simply $x$ for $i(x)$. For any $x \in \mathcal{B}(\mathbf{H}, k)$, the associated filtrations on $H:=\mathbf{H}(k)$ and $\mathfrak{h}:=\mathfrak{h}(k)$ satisfy the following ([1, (1.9.1)]):

$$
\begin{aligned}
H_{x, r} & =\mathbf{G}(E)_{x, r} \cap H=G_{x, r} \cap H \quad \text { for } r>0, \\
\mathfrak{h}_{x, r} & =\mathfrak{g}(E)_{x, r} \cap \mathfrak{h}=\mathfrak{g}_{x, r} \cap \mathfrak{h} \quad \text { for any } r \in \tilde{\mathbb{R}} .
\end{aligned}
$$

For a $k$-torus $\mathbf{T} \subset \mathbf{H}$ which splits over $E$, we have $\mathcal{A}(\mathbf{H}, \mathbf{T}, k)=\mathcal{A}(\mathbf{G}, \mathbf{T}, E) \cap$ $\mathcal{B}(\mathbf{H}, k)$.

2.3. If $\overrightarrow{\mathbf{G}}=\left(\mathbf{G}^{0}, \cdots, \mathbf{G}^{d}=\mathbf{G}\right)$ is a tamely ramified twisted Levi sequence, we can and will fix a sequence of embeddings

$$
\mathcal{B}\left(\mathbf{G}^{0}, k\right) \hookrightarrow \mathcal{B}\left(\mathbf{G}^{1}, k\right) \hookrightarrow \mathcal{B}\left(\mathbf{G}^{2}, k\right) \hookrightarrow \cdots \hookrightarrow \mathcal{B}\left(\mathbf{G}^{d}, k\right) .
$$

We will identify $\mathcal{B}\left(\mathbf{G}^{i}, k\right)$ with a subset of $\mathcal{B}\left(\mathbf{G}^{j}, k\right)$ for $i \leq j$. Moreover, for $x \in \mathcal{B}\left(\mathbf{G}^{i}, k\right)$ and $r \in \tilde{\mathbb{R}}$, we have

$$
\begin{aligned}
\mathfrak{g}_{x, r}^{i} & =\mathfrak{g}^{j}(E)_{x, r} \cap \mathfrak{g}^{i}=\mathfrak{g}_{x, r}^{j} \cap \mathfrak{g}^{i}, \\
G_{x, r}^{i} & =\mathbf{G}^{j}(E)_{x, r} \cap G^{i}=G_{x, r}^{j} \cap G^{i} \quad \text { if } r>0 .
\end{aligned}
$$

From now on, we say that a semisimple element $\Gamma \in \mathfrak{g}$ splits over a finite extension $E$ if $\Gamma$ lies on a $k$-torus which splits over $E$.

Lemma 2.4. Let $\Gamma \in \mathfrak{g}$ be a semisimple element which splits over a tamely ramified finite extension $E$ of $k$. Set $\mathbf{H}:=\mathbf{C}_{\mathbf{G}}(\Gamma)$, the centralizer of $\Gamma$ in $\mathbf{G}$. Then $(\mathbf{H}, \mathbf{G})$ is an E-split tamely ramified twisted Levi sequence.

Proof. Without loss of generality, we may assume that $\Gamma$ is in a $k$-split torus $\mathfrak{t}$. By (7.1) and (7.2) of [38, $\mathbf{H}$ is connected and reductive. Since $\mathfrak{h}$ is reductive, $\mathfrak{z}_{\mathfrak{h}} \subset \mathfrak{t}$, and thus $\mathfrak{z}_{\mathfrak{h}}$ is a $k$-split subtorus of $\mathfrak{t}$. Combining this with the fact that $\mathbf{H}$ is the centralizer of $\mathfrak{z}_{\mathfrak{h}}$ in $\mathbf{G}$, we conclude that $\mathbf{H}$ is a $k$-Levi subgroup of $\mathbf{G}$.

Remarks 2.5.

(1) Note that if $\Gamma=0$, then $\mathbf{H}=\mathbf{G}$.

(2) The above lemma is not valid if $\Gamma$ is replaced by a semisimple element of $G$. For example, in $\mathbf{S p}_{4}$, the centralizer of a semisimple group element can be $\mathbf{S L}_{2} \times \mathbf{S L}_{2}$. 
2.6. Let $(\mathbf{H}, \mathbf{G})$ be a tamely ramified twisted Levi sequence. For $X \in \mathfrak{g}$, denote the $H$-orbit ${ }^{H} X$ of $X$ by $\mathcal{O}_{X}^{H}$. For simplicity, we write $\mathcal{O}_{X}$ for $\mathcal{O}_{X}^{G}$. In general, we use the notation $\mathcal{O}^{H}$ to denote $H$-orbits. If $X \in \mathfrak{h}, \mathcal{O}(H, X)$ denotes the set of all $H$-orbits whose closure in $\mathfrak{h}$ contains $X$. We write $\mathcal{O}(X):=\mathcal{O}(G, X)$.

When $X \in \mathfrak{g}$ is semisimple, $\mathcal{O}(X)$ is described in [15, §2]. In the situation of Lemma 2.4. write $\Gamma=X$ and $H=C_{G}(\Gamma)$. Then

$$
\mathcal{O}(\Gamma)=\left\{\mathcal{O}_{\Gamma+n} \mid n \in \mathcal{O}^{H} \in \mathcal{O}(H, 0)\right\} .
$$

Note that $\Gamma+n$ is already in the form of a Jordan decomposition. The map $\mathcal{O}_{\Gamma+n} \rightarrow \mathcal{O}_{n}, n \in \mathcal{O}(H, 0)$, induces a bijection of $\mathcal{O}(\Gamma)$ with $\mathcal{O}(H, 0)$, the set of nilpotent $H$-orbits in $\mathfrak{h}$.

\section{Admissible Sequences And lattices in $\mathfrak{g}$ AND $\mathfrak{g}^{*}$}

We recall some definitions from [9] and [38].

Definition 3.1. Let $E / k$ be a tamely ramified extension, and let $\mathbf{T}$ be an $E$-split maximal $k$-torus in $\mathbf{G}$. Let $\Phi=\Phi(\mathbf{G}, \mathbf{T}, E)$ be the corresponding root system. Then, a function $f: \Phi \cup\{0\} \longrightarrow \tilde{\mathbb{R}}$ is concave if for every nonempty finite subset $\left\{a_{i}\right\} \subset \Phi \cup\{0\}$ such that $\sum a_{i} \in \Phi \cup\{0\}$, we have

$$
f\left(\sum_{i} a_{i}\right) \leq \sum_{i} f\left(a_{i}\right)
$$

We keep the notation from the above definition. If $x \in \mathcal{A}(\mathbf{G}, \mathbf{T}, E)$ and $f$ is a concave function on $\Phi(\mathbf{G}, \mathbf{T}, E) \cup\{0\}$, there are a group $\mathbf{G}(E)_{x, f}$ and a lattice $\mathfrak{g}(E)_{x, f}$ associated to $x$ and $f$ (see [3, 38] for details). If $f$ is $\operatorname{Gal}(E / k)$-invariant and $x \in \mathcal{A}(\mathbf{G}, \mathbf{T}, E)^{\operatorname{Gal}(E / k)}$, we can define

$$
\begin{aligned}
G_{x, f} & :=\mathbf{G}(E)_{x, f}^{\operatorname{Gal}(E / k)} \cap G_{x, 0}, \\
\mathfrak{g}_{x, f} & :=\mathfrak{g}(E)_{x, f}^{\operatorname{Gal}(E / k)} .
\end{aligned}
$$

If $f$ is positive and $E / k$ is tame, then $G_{x, f}=\mathbf{G}(E)_{x, f}^{\operatorname{Gal}(E / k)}$.

Definition 3.2. A sequence $\vec{u}:=\left(u_{0}, \cdots, u_{d}\right)$ in $\tilde{\mathbb{R}}$ is admissible if for some $0 \leq$ $c \leq d$, we have

$$
0 \leq u_{0}=u_{1}=\cdots=u_{c} \quad \text { and } \quad \frac{1}{2} u_{c} \leq u_{c+1} \leq \cdots \leq u_{d} .
$$

3.3. Let $E$ be a tamely ramified Galois extension of $k$. Let $\overrightarrow{\mathbf{G}}=\left(\mathbf{G}^{0}, \cdots, \mathbf{G}^{d}=\mathbf{G}\right)$ be an $E$-split twisted Levi sequence, and let $\vec{u}=\left(u_{0}, \cdots, u_{d}\right)$ be an admissible sequence. Then we can associate to $\vec{u}$ a $\operatorname{Gal}(E / k)$-invariant, concave function $f_{\vec{u}}$ on $\Phi(\mathbf{G}, \mathbf{T}, E)$ as follows: for an $E$-split maximal $k$-torus $\mathbf{T} \subseteq \mathbf{G}^{0}$, define

$$
f_{\vec{u}}(a)= \begin{cases}u_{0} & \text { if } a \in \Phi\left(\mathbf{G}^{0}, \mathbf{T}, E\right) \cup\{0\}, \\ u_{i} & \text { if } a \in \Phi\left(\mathbf{G}^{i}, \mathbf{T}, E\right) \backslash \Phi\left(\mathbf{G}^{i-1}, \mathbf{T}, E\right) \text { for } i>0 .\end{cases}
$$

For $x \in \mathcal{A}(\mathbf{G}, \mathbf{T}, k)$, let

$$
\vec{G}_{x, \vec{u}}:=G_{x, f_{\vec{u}}}, \quad \quad \overrightarrow{\mathfrak{g}}_{x, \vec{u}}:=\mathfrak{g}_{x, f_{\vec{u}}} .
$$

These are well defined independent of the choice of $\mathbf{T}$ with $x \in \mathcal{A}(\mathbf{G}, \mathbf{T}, k)$. Moreover, if $\vec{u}$ is nondecreasing, we have

$$
\vec{G}_{x, \vec{u}}=G_{x, u_{0}}^{0} G_{x, u_{1}}^{1} \cdots G_{x, u_{d}}^{d}, \quad \overrightarrow{\mathfrak{g}}_{x, \vec{u}}=\mathfrak{g}_{x, u_{0}}^{0}+\mathfrak{g}_{x, u_{1}}^{1}+\cdots+\mathfrak{g}_{x, u_{d}}^{d} .
$$


3.4. Hypotheses. We list the hypotheses used in this paper. They are labeled by $(\mathrm{H} k),(\mathrm{HB}),(\mathrm{HGT})$ and $(\mathrm{H \mathcal {N }})$, respectively. We will state explicitly whenever these hypotheses are necessary.

$(\mathrm{H} k)$ The residue characteristic $p$ is large enough (depending on $\mathbf{G}$ and $\nu(p)$ ) such that the following hold.

(1) The exponential map (resp. the logarithmic map) is defined on the subset $\mathfrak{g}_{0^{+}}$of $\mathfrak{g}$ (resp. $G_{0^{+}}$of $G$ ), and for a tamely ramified finite Galois extension $E$ over $k$, an $E$-split maximal $k$-torus $\mathbf{T}$ of $\mathbf{G}, x \in \mathcal{A}(\mathbf{G}, \mathbf{T}, k)$, and a $\operatorname{Gal}(E / k)$-invariant concave function $f$ on $\Phi(\mathbf{G}, \mathbf{T}, E) \cup\{0\}$ with $f(0)>0$, we have $\exp \left(\mathfrak{g}_{x, f}\right)=G_{x, f}\left(\right.$ resp. $\left.\log \left(G_{x, f}\right)=\mathfrak{g}_{x, f}\right)$.

(2) For any $x \in \mathcal{B}(\mathbf{G}, k), X \in \mathfrak{g}_{x, 0^{+}}$and $Y \in \mathfrak{g}_{x, 0}, \frac{1}{p}(\operatorname{ad} X)^{p-1}(Y) \in \mathfrak{g}_{x, 0^{+}}$.

(HB) $\mathbf{G}$ satisfies the condition in Proposition 4.1 in [4].

(HGT) Every maximal $k$-torus $\mathbf{T}$ in $\mathbf{G}$ splits over a tamely ramified Galois extension, and for any $r \in \mathbb{R}$, any nontrivial coset in $\mathfrak{t}_{r}$ modulo $\mathfrak{t}_{r}+$ contains a good element (as defined in Definition 4.2) of depth $r$.

$(\mathrm{H} \mathcal{N})$ For any tamely ramified twisted Levi subgroup $\mathbf{H}$ of $\mathbf{G}$, the hypotheses in [12, $\S 4.2]$ are valid.

Remark 3.5. The condition in Proposition 4.1 of 4 requires that either $\mathbf{G}$ be a form of $\mathrm{GL}_{n}$ or the absolute Dynkin diagram of $\mathbf{G}$ have no bonds of order $p$ and that $p$ not divide $2 \mathrm{k}(\mathbf{G})\left|\pi_{1}\left(\mathbf{G}^{\prime}\right)\right|$ (see [4, Proposition 4.1 for notation). One sees easily that this is satisfied if $p$ is large enough.

One can use the Campbell-Hausdorff formula to determine a sufficient condition on $k$ for $(\mathrm{H} k)$ to hold (see [24, Proposition 3.1.1).

In [12], under some hypotheses on $\mathbf{G}$ and $k$ (see [12, $\S 4.2$ ), DeBacker gives a parameterization of nilpotent orbits in $\mathfrak{g}$ via Bruhat-Tits theory (see Theorem 5.6.1 of [12]). He uses this parameterization to get a homogeneity result in [11]. We need the hypothesis $(\mathrm{HN})$ to use the results of [11] and [12]. We refer the reader to [12] for precise statements. Again, if $p$ is large enough, $(\mathrm{HN})$ is valid.

Remark 3.6. If (HB) is satisfied, there is a $\bar{k}$-valued, nondegenerate, $\mathbf{G}(\bar{k})$-invariant, symmetric, bilinear form B on $\mathfrak{g}$ satisfying the following (see the proof of [4, (4.1)]): for any tamely ramified finite extension $E$ of $k, \mathrm{~B}$ induces an $E$-valued $E$-bilinear form on $\mathfrak{g}(E)$ such that

(1) we can identify $\mathfrak{g}^{*}(E)_{x, r}$ with $\mathfrak{g}(E)_{x, r}$ via the map $\Omega: \mathfrak{g}(E) \rightarrow \mathfrak{g}^{*}(E)$ defined by $\Omega(X)(Y)=\mathrm{B}(X, Y)$;

(2) if $\mathbf{T}$ is a maximal $E$-split torus and $\left(\mathbf{T},\left\{X_{\alpha}\right\}\right)$ is a Chevalley splitting, then we have

(i) $\mathrm{B}\left(X_{\alpha}, X_{\beta}\right)=0$ unless $\alpha+\beta=0$,

(ii) $\mathrm{B}\left(X_{\alpha}, X_{-\alpha}\right) \neq 0$ and has valuation 0 ,

(iii) $\mathfrak{g}=\mathfrak{z}_{\mathfrak{g}} \oplus \mathfrak{g}^{\text {der }}$ is an orthogonal decomposition with respect to B;

(3) for any Levi subgroup $\mathbf{M}$ of $\mathbf{G}$ which splits over a tamely ramified extension, $\mathrm{B} \mid \mathfrak{m} \times \mathfrak{m}$ satisfies (1) and (2).

The above (HB) implies the corresponding hypothesis (HB) (labeled in the same way) in [27. Whenever we assume (HB), we denote the associated bilinear form by B. For more discussion on sufficient conditions for the above hypotheses, we refer to [27]. 
3.7. Let $\vec{u}=\left(u_{0}, \cdots, u_{d}\right)$ be a sequence of real numbers which is not necessarily admissible. We can still define $f_{\vec{u}}$ and a subset $\overrightarrow{\mathfrak{g}}_{x, \vec{u}}=\mathfrak{g}_{x, f_{\vec{u}}}$ of $\mathfrak{g}$ in a similar fashion as in 33.3 . Suppose (HB) is valid.

For each $i=0, \cdots, d-1$, let $\mathfrak{g}^{i \perp}$ be the orthogonal complement of $\mathfrak{g}^{i}$ in $\mathfrak{g}^{i+1}$ with respect to $\mathrm{B}$, and let $\mathfrak{g}_{\perp}^{i}$ be the orthogonal complement of $\mathfrak{g}^{i}$ in $\mathfrak{g}$ with respect to B. Then, we have

$$
\mathfrak{g}^{i+1}=\mathfrak{g}^{i} \oplus \mathfrak{g}^{i \perp}, \quad \mathfrak{g}=\mathfrak{g}^{i} \oplus \mathfrak{g}_{\perp}^{i}
$$

and

$$
\mathfrak{g}=\mathfrak{g}^{0} \oplus \mathfrak{g}^{0 \perp} \oplus \cdots \oplus \mathfrak{g}^{d-1 \perp}, \quad \mathfrak{g}_{\perp}^{i}=\mathfrak{g}^{i \perp} \oplus \cdots \oplus \mathfrak{g}^{d-1 \perp} .
$$

For $x \in \mathcal{B}\left(\mathbf{G}^{i}, k\right)$ and $r \in \tilde{\mathbb{R}}$, let $\mathfrak{g}_{x, r}^{i \perp}=\mathfrak{g}_{x, r}^{i+1} \cap \mathfrak{g}^{i \perp}$. Then, for $x \in \mathcal{B}\left(\mathbf{G}^{0}, k\right)$, we can write $\overrightarrow{\mathfrak{g}}_{x, \vec{u}}$ more explicitly:

$$
\overrightarrow{\mathfrak{g}}_{x, \vec{u}}=\mathfrak{g}_{x, f_{\vec{u}}}=\mathfrak{g}_{x, u_{0}}^{0} \oplus \mathfrak{g}_{x, u_{1}}^{0 \perp} \oplus \cdots \oplus \mathfrak{g}_{x, u_{d}}^{d-1 \perp} .
$$

\section{Definition 3.8.}

(1) Define an involution $*$ on $\tilde{\mathbb{R}}$ as follows: for $r \in \mathbb{R}, r^{*}:=(-r)^{+}$and $\left(r^{+}\right)^{*}:=$ $-r$.

(2) For a sequence $\vec{r}=\left(r_{1}, \cdots, r_{d}\right)$ of real numbers, define $\vec{r}^{*}$ as $\left(r_{1}^{*}, \cdots, r_{d}^{*}\right)$.

(3) Assume (HB) is valid. When we identify $\mathfrak{g}$ and $\mathfrak{g}^{*}$ via $\mathrm{B}$, the dual $\left(\mathfrak{g}_{x, r}\right)^{*} \subset$ $\mathfrak{g}$ of $\mathfrak{g}_{x, r}$ with respect to B is $\mathfrak{g}_{x, r^{*}}$. That is, $\left(\mathfrak{g}_{x, r}\right)^{*}=\left\{Y \in \mathfrak{g} \mid \mathrm{B}\left(Y, \mathfrak{g}_{x, r}\right) \subset\right.$ $\left.\mathfrak{p}_{k}\right\}=\mathfrak{g}_{x, r^{*}}$. Note that $\left(\mathfrak{g}_{x, r}\right)^{*} \subset \mathfrak{g}$ while $\mathfrak{g}_{x, r}^{*} \subset \mathfrak{g}^{*}$. Generalizing this, for any subset $\mathcal{L} \subset \mathfrak{g}$, we define the dual $\mathcal{L}^{*}$ of $\mathcal{L}$ in $\mathfrak{g}$ as

$$
\mathcal{L}^{*}:=\left\{Y \in \mathfrak{g} \mid \mathrm{B}(Y, \mathcal{L}) \subset \mathfrak{p}_{k}\right\}
$$

Remark 3.9. Let $\overrightarrow{\mathbf{G}}:=\left(\mathbf{G}^{0}, \cdots, \mathbf{G}^{d}\right)$ be a tamely ramified twisted Levi sequence. Let $x \in \mathcal{B}\left(\mathbf{G}^{0}, k\right)$, and let $\vec{u}:=\left(u_{0}, \cdots, u_{d}\right)$ be an admissible sequence. Assume the hypothesis (HB) is valid. Then, we have ([27])

$$
\left(\overrightarrow{\mathfrak{g}}_{x, \vec{u}}\right)^{*}=\overrightarrow{\mathfrak{g}}_{x, \vec{u}^{*}}=\mathfrak{g}_{x, u_{0}^{*}}^{0} \oplus \mathfrak{g}_{x, u_{1}^{*}}^{0 \perp} \oplus \cdots \oplus \mathfrak{g}_{x, u_{d}^{*}}^{d-1 \perp} .
$$

Definition 3.10. Suppose (HB) is valid. Let $L$ be an open compact subgroup of $G$ with $L=\exp (\mathcal{L})$ for some lattice $\mathcal{L}$ in $\mathfrak{g}$. Let $\chi$ be a character of $L$. If there is a $\gamma \in \mathfrak{g}$ such that $\chi(g)=\Lambda(\mathrm{B}(\gamma, \log (g)))$, we say that $\chi$ is represented by $\gamma$, and we write $\chi_{\gamma}$ for $\chi$. In this case, any element $\gamma^{\prime}$ in the coset $\mathcal{S}=\gamma+\mathcal{L}^{*} \in \mathfrak{g} / \mathcal{L}^{*}$ represents $\chi$. We call $\mathcal{S}$ the dual blob of $(L, \chi)$.

Remark 3.11. It is possible that $\gamma \in \mathfrak{g}$ represents characters on different groups, say $L_{1}$ and $L_{2}$. However, since those characters coincide on $L_{1} \cap L_{2}$, we will use $\chi_{\gamma}$ to denote both characters when there is no confusion.

For a sufficient condition for $\gamma \in \mathfrak{g}$ to represent a character of $\vec{G}_{x, \vec{u}}$, we refer to [27. (3.3.4)].

The proof of the following lemma is similar to that of Lemma 3.1 in [21, p. 17].

Lemma 3.12. Suppose $(\mathrm{HB})$ and $(\mathrm{H} k)$ are valid. For $i=1,2$, let $L_{i}$ be an open compact subgroup with $L_{i}=\exp \left(\mathcal{L}_{i}\right)$ for some $\mathcal{L}_{i} \subset \mathfrak{g}$. Let $\chi_{i}$ be a character of $L_{i}$ with dual blob $\gamma_{i}+\mathcal{L}_{i}^{*}$. Suppose $\chi_{1}=\chi_{2}$ on $L_{1} \cap L_{2}$. Then, $\left(\gamma_{1}+\mathcal{L}_{1}^{*}\right) \cap\left(\gamma_{2}+\mathcal{L}_{2}^{*}\right) \neq \emptyset$.

Proof. Since both $\gamma_{1}$ and $\gamma_{2}$ represent $\chi_{1}\left|\left(L_{1} \cap L_{2}\right)=\chi_{2}\right|\left(L_{1} \cap L_{2}\right)$, we have $\gamma_{1}+$ $\left(\mathcal{L}_{1} \cap \mathcal{L}_{2}\right)^{*}=\gamma_{2}+\left(\mathcal{L}_{1} \cap \mathcal{L}_{2}\right)^{*}$. Now, the lemma follows from $\left(\mathcal{L}_{1} \cap \mathcal{L}_{2}\right)^{*}=\mathcal{L}_{1}^{*}+\mathcal{L}_{2}^{*}$. 


\section{Semisimple ELEMENTS}

4.1. Depth functions and good elements. Recall that the depth function $\mathrm{d}: \mathcal{B}(G) \times \mathfrak{g} \longrightarrow \mathbb{R}$ is defined as follows: for $X \in \mathfrak{g}$ and $x \in \mathcal{B}(G)$, let $\mathrm{d}(x, X)=r$ be the depth of $X$ in the $x$-filtration, that is, $r$ is the unique real number such that $X \in \mathfrak{g}_{x, r} \backslash \mathfrak{g}_{x, r^{+}}$. We also define

$$
\mathrm{d}(X)=\sup _{x \in \mathcal{B}(G)} \mathrm{d}(x, X) .
$$

Note that if $\mathrm{d}(X)<\infty$, then the depth $\mathrm{d}(X)$ of $X$ is the unique $r$ in $\mathbb{R}$ such that $X \in \mathfrak{g}_{r} \backslash \mathfrak{g}_{r^{+}}$. Moreover, $\mathrm{d}$ is locally constant on $\mathfrak{g} \backslash \mathcal{N}$, and it is $\infty$ on $\mathcal{N}$ (see 2, (3.3.7)]). If $E$ is a finite extension of $k$, we can also define a depth function $\mathrm{d}^{E}$ on $\mathcal{B}(\mathbf{G}, E) \times \mathfrak{g}(E)$. If $E$ is tamely ramified over $k$, thanks to our normalization of valuation, we observe that for any $x \in \mathcal{B}(G)$ and $X \in \mathfrak{g}, \mathrm{d}(x, X)=\mathrm{d}^{E}(x, X)$ and $\mathrm{d}(X)=\mathrm{d}^{E}(X)$ (see [3, (2.2.5)]). Hence we may omit the superscript ${ }^{E}$ in that case. We remark that if $X$ has Jordan decomposition $X_{s}+X_{n}$, with semisimple part $X_{s}$, then $\mathrm{d}(X)=\mathrm{d}\left(X_{s}\right)([2,(3.3 .8)])$.

Let $\mathbf{T}$ be a maximal $k$-torus in $\mathbf{G}$, and let $\mathfrak{t}$ be its Lie algebra. Then $T$ and $\mathfrak{t}$ have the following filtrations: for $r \in \mathbb{R}$

$$
\mathfrak{t}_{r}=\left\{\Gamma \in \mathfrak{t} \mid \nu(d \chi(\Gamma)) \geq r \text { for all } \chi \in \mathbf{X}^{*}(\mathbf{T})\right\}
$$

and for $r>0$,

$$
T_{r}=\left\{t \in T \mid \nu(\chi(t)-1) \geq r \text { for all } \chi \in \mathbf{X}^{*}(\mathbf{T})\right\} .
$$

Note that if $\mathbf{T}$ is $k$-split, the lattice $\mathfrak{t}_{r}$ coincides with the lattice $\mathfrak{t}(k)_{r}$ of 1.3 . The following definition is from $[4, \S 5]$.

Definition 4.2. Let $\mathbf{T}$ be a maximal $k$-torus in $\mathbf{G}$ which splits over a tamely ramified Galois extension of $k$, and let $\mathfrak{t}$ be its Lie algebra.

(1) If $\Gamma \in \mathfrak{t}_{r} \backslash \mathfrak{t}_{r+}$, we say that $\Gamma$ is of depth $r$ with respect to $\mathbf{T}$, and we write $\mathrm{d}_{\mathbf{T}}(\Gamma)=r$

(2) Let $\Gamma \in \mathfrak{t}$ be of depth $r$. Then $\Gamma$ is called good with respect to $\mathbf{T}$ if for every root $\alpha$ of $\mathbf{G}$ with respect to $\mathbf{T}, d \alpha(\Gamma)$ is either zero or has valuation $r$.

Note that $0 \in \mathfrak{g}$ is a good element of depth $\infty$. We remark that the depth and the goodness of a semisimple element do not depend on the choice of $\mathbf{T}(\underline{4},(5.1)])$.

4.3. We recall some useful facts about degenerate cosets (see [2, (3.2.6)] and [30, $(6.3)])$.

(1) Assume $X \in \mathfrak{g}_{x, r} \cap \mathfrak{g}_{r^{+}}$. Then,

(i) $X+\mathfrak{g}_{x, r^{+}}$contains a nilpotent element and

(ii) there is a $y \in \mathcal{B}(\mathbf{G}, k)$ such that $X+\mathfrak{g}_{x, r^{+}} \subset \mathfrak{g}_{y, r^{+}}$.

(2) Let $s \in \mathbb{R}$. Then, $\mathfrak{g}_{s}=\bigcap_{x \in \mathcal{B}(\mathbf{G}, k)}\left(\mathcal{N}+\mathfrak{g}_{x, s}\right)$.

(3) If $x \in \mathcal{B}(\mathbf{G}, k)$ and $s \in \mathbb{R}$, then $\mathfrak{g}_{x, s} \cap \mathfrak{g}_{s^{+}}=\left(\mathcal{N} \cap \mathfrak{g}_{x, s}\right)+\mathfrak{g}_{x, s^{+}}$.

4.4. Let $\mathbf{T}$ be a maximal $k$-torus in $\mathbf{G}$ which splits over a tamely ramified Galois extension $E$. Then we observe the following:

(1) Let $\Phi:=\Phi(\mathbf{G}, \mathbf{T}, E)$. Then we have

$$
\mathfrak{g}(E)=\mathfrak{t}(E) \oplus \sum_{\alpha \in \Phi} \mathfrak{g}_{\alpha}(E) .
$$


Let $\Gamma \in \mathfrak{t}$ be a semisimple element. Let $\mathbf{H}:=\mathbf{C}_{\mathbf{G}}(\Gamma)$ be the centralizer of $\Gamma$ in $\mathbf{G}$. The Lie algebra $\mathfrak{h}(E)$ can be expressed as follows [38, (7.1)]:

$$
\mathfrak{h}(E)=\mathfrak{t}(E) \oplus \sum_{\alpha \in \Phi, d \alpha(\Gamma)=0} \mathfrak{g}_{\alpha}(E)
$$

(2) If $\Gamma \notin \mathfrak{z}_{\mathfrak{g}}$ is a good element of depth $r$, then for any $\gamma \in \mathfrak{z}_{\mathfrak{g}}$ with $\mathrm{d}(\gamma) \geq r$, $\Gamma+\gamma$ is also a good element of depth $r$.

Lemma 4.5. Let $\mathbf{T}$ be a maximal $k$-torus in $\mathbf{G}$ which splits over a tamely ramified Galois extension $E$. Let $\gamma=\gamma_{1}, \cdots, \gamma_{n} \in \mathfrak{t}$ be good elements of depth $b=b_{1}, \cdots, b_{n}$, respectively. Let $\mathbf{H}^{0}:=\mathbf{G}$ and $\mathbf{H}^{i}:=\mathbf{C}_{\mathbf{H}^{i-1}}\left(\gamma_{i}\right)$.

(1) Let $X_{1}, X_{2} \in \gamma+\mathfrak{h}_{b^{+}}^{1}$. If $g \in G$ is such that ${ }^{g} X_{1}=X_{2}$, then $g \in H^{1}$.

(2) Let $X \in \gamma+\mathfrak{h}_{b^{+}}^{1}$. Then $C_{G}(X) \subset H^{1}$.

(3) Suppose each $\gamma_{i}$ is a good element in $\mathbf{H}^{i-1}$, and suppose $b_{1}<b_{2}<\cdots<b_{n}$. Fix $i \in\{0,1, \cdots, d\}$ and let $\gamma^{i}=\gamma_{1}+\gamma_{2}+\cdots+\gamma_{i}$. Let $Y_{1}, Y_{2} \in \gamma^{i}+\mathfrak{h}_{b_{i}^{+}}^{i}$. If ${ }^{g} Y_{1}=Y_{2}$ for some $g \in G$, then $g \in H^{i}$. Moreover, $\mathbf{H}^{i}=\mathbf{C}_{\mathbf{G}}\left(\gamma^{i}\right)$

Proof. (1) is [26, (2.3.6)], and (2) follows from (1). For the first statement of (3), if $i=1$, it is (1). Assume the statement is true for $i$. Let $Y_{1}, Y_{2} \in \gamma^{i+1}+\mathfrak{h}_{b_{i+1}^{+}}^{i+1}$. Suppose ${ }^{g} Y_{1}=Y_{2}$ for some $g \in G$. Since $Y_{1}, Y_{2} \in \gamma^{i}+\mathfrak{h}_{b_{i}^{+}}^{i}$, we have $g \in H^{i}$ by the induction hypothesis and ${ }^{g}\left(Y_{1}-\gamma^{i}\right)=Y_{2}-\gamma^{i}$. Since $Y_{1}-\gamma^{i}, Y_{2}-\gamma^{i} \in \gamma_{i+1}+\mathfrak{h}_{b_{i+1}^{+}}^{i+1}$ and $\gamma_{i+1}$ is $\mathbf{H}^{i}$-good, we have $g \in H^{i+1}$. For the second statement, $\mathbf{H}^{i} \subset \mathbf{C}_{\mathbf{G}}\left(\gamma^{i}\right)$ is obvious, and $\mathbf{C}_{\mathbf{G}}\left(\gamma^{i}\right) \subset \mathbf{H}^{i}$ follows from the first with $Y_{1}=Y_{2}=\gamma$.

Lemma 4.6. Let $\mathbf{T}$ be a maximal $k$-torus in $\mathbf{G}$ which splits over a tamely ramified Galois extension $E$. Let $\gamma_{1}, \gamma_{2} \in \mathfrak{t}$ be good elements of depth $b_{1}, b_{2}$, respectively. Let $\mathbf{H}:=\mathbf{C}_{\mathbf{G}}\left(\gamma_{1}\right)$.

(1) Suppose $b=b_{1}=b_{2}$ and $\gamma_{1} \equiv \gamma_{2}\left(\bmod \mathfrak{t}_{b^{+}}\right)$. Then $C_{G}\left(\gamma_{1}\right)=C_{G}\left(\gamma_{2}\right)$.

(2) Suppose $b_{1}<b_{2}$ and $\gamma_{1}, \gamma_{2} \in \mathfrak{z h}$. Then $\gamma_{1}+\gamma_{2}$ is also a G-good element of depth $b_{1}$.

Proof. (1) Note that $\gamma_{1}, \gamma_{2} \in \gamma_{1}+\mathfrak{h}_{b^{+}}$. Applying Lemma4.5(2), we have $C_{G}\left(\gamma_{1}\right) \subset$ $C_{G}\left(\gamma_{2}\right)$. Similarly, $C_{G}\left(\gamma_{2}\right) \subset C_{G}\left(\gamma_{1}\right)$. Hence $C_{G}\left(\gamma_{1}\right)=C_{G}\left(\gamma_{2}\right)$.

(2) Write $\Gamma=\gamma_{1}+\gamma_{2}$. Let $\Phi=\Phi(\mathbf{G}, \mathbf{T}, E)$ be the set of E-rational T-roots in G. Let $\alpha \in \Phi$. Since $\mathbf{H} \subset \mathbf{C}_{\mathbf{G}}\left(\gamma_{2}\right)$, by 4.4 , we see that if $d \alpha\left(\gamma_{1}\right)=0$, then $d \alpha\left(\gamma_{2}\right)=0$. Combining this with $\nu\left(d \alpha\left(\gamma_{2}\right)\right) \geq b_{2}>b_{1}$, we see that $d \alpha\left(\gamma_{1}+\gamma_{2}\right)=0$ or $\nu\left(d \alpha\left(\gamma_{1}+\gamma_{2}\right)\right)=\min \left(\nu\left(d \alpha\left(\gamma_{1}\right)\right), \nu\left(d \alpha\left(\gamma_{2}\right)\right)\right)=\nu\left(d \alpha\left(\gamma_{1}\right)\right)=b_{1}$. Hence $\gamma_{1}+\gamma_{2}$ is a good element of depth $b_{1}$.

Proposition 4.7. Suppose (HGT) is valid. Let $\gamma \in \mathfrak{g}$ be a semisimple element which splits over a tamely ramified Galois extension E. Then, $\gamma$ can be written as

$$
\gamma=\gamma_{b_{1}}+\gamma_{b_{2}}+\cdots+\gamma_{b_{n}}+\gamma_{\circ}
$$

such that

(1) each $\gamma_{b_{i}}, i=1, \cdots, n$, is a G-good element of depth $b_{i}$, and $\gamma_{\circ}$ is a semisimple element with $\mathrm{d}\left(\gamma_{0}\right) \geq 0$,

(2) $b_{1}<b_{2}<\cdots<b_{n}<0$, and

(3) $\mathbf{H}^{n} \subsetneq \mathbf{H}^{n-1} \subsetneq \cdots \subsetneq \mathbf{H}^{1} \subseteq \mathbf{G}$ where $\mathbf{H}^{1}=\mathbf{C}_{\mathbf{G}}\left(\gamma_{b_{1}}\right)$ and $\mathbf{H}^{i}=\mathbf{C}_{\mathbf{H}^{i-1}}\left(\gamma_{b_{i}}\right)$. 
Moreover, if $\gamma=\gamma_{b_{1}^{\prime}}^{\prime}+\gamma_{b_{2}^{\prime}}^{\prime}+\cdots+\gamma_{b_{n^{\prime}}^{\prime}}^{\prime}+\gamma_{\circ}^{\prime}$ is another expression satisfying (1)-(3) with $\mathbf{H}^{\prime 1}=\mathbf{C}_{\mathbf{G}}\left(\gamma_{b_{1}^{\prime}}^{\prime}\right)$ and $\mathbf{H}^{i}=\mathbf{C}_{\mathbf{H}^{\prime i-1}}\left(\gamma_{b_{i}^{\prime}}^{\prime}\right)$, then we have $n=n^{\prime}, b_{i}=b_{i}^{\prime}$ and $\mathbf{H}^{i}=\mathbf{H}^{i}$

Proof. Let $\mathfrak{t}$ be a maximal $k$-torus with $\gamma \in \mathfrak{t}$ which splits over $E$. If $\mathrm{d}(\gamma) \geq 0$, $\gamma=\gamma_{\circ}$ already satisfies (1)-(3). Suppose $a_{1}=\mathrm{d}(\gamma)<0$. By (HGT), $\gamma+\mathfrak{t}_{a_{1}^{+}}$ contains a good element, say $\tilde{\gamma}_{a_{1}}$ of depth $a_{1}$. Then $\gamma=\tilde{\gamma}_{a_{1}}+\left(\gamma-\tilde{\gamma}_{a_{1}}\right)$ with $a_{2}=\mathrm{d}\left(\gamma-\tilde{\gamma}_{a_{1}}\right)>\mathrm{d}(\gamma)$. Applying the above process for $\gamma-\tilde{\gamma}_{a_{1}}$, we find a $G$ good element $\tilde{\gamma}_{a_{2}} \in \gamma-\tilde{\gamma}_{a_{1}}+\mathfrak{t}_{a_{2}^{+}}$such that $\gamma=\tilde{\gamma}_{a_{1}}+\tilde{\gamma}_{a_{2}}+\left(\gamma-\tilde{\gamma}_{a_{1}}-\tilde{\gamma}_{a_{2}}\right)$ and $a_{3}=\mathrm{d}\left(\gamma-\tilde{\gamma}_{a_{1}}-\tilde{\gamma}_{a_{2}}\right)<\mathrm{d}\left(\gamma-\tilde{\gamma}_{a_{1}}\right)$. Repeatedly, we have

$$
\gamma=\tilde{\gamma}_{a_{1}}+\tilde{\gamma}_{a_{2}}+\cdots+\tilde{\gamma}_{a_{m}}+\gamma_{\circ}
$$

where $\tilde{\gamma}_{a_{i}}$ is a $G$-good element of depth $a_{i}$ with $a_{1}<a_{2}<\cdots<a_{m}<0$ and $\mathrm{d}\left(\gamma_{0}\right) \geq 0$. This procedure is finite because $\mathrm{d}(\mathfrak{t}) \subset \frac{1}{e(E / k)} \mathbb{Z}$. Put $a_{m+1}=0$ and $\tilde{\gamma}_{a_{m+1}}=\gamma_{\circ}$.

Set $S:=\left\{a_{1}, a_{2}, \cdots, a_{m+1}\right\}$, and for $a, b \in \tilde{\mathbb{R}}$, set $\tilde{\gamma}_{a, b}:=\sum_{a \leq a_{j}<b} \tilde{\gamma}_{a_{j}}$. We find a subsequence $b_{1}=a_{1}<b_{2}<\cdots<b_{n}<b_{n+1}=a_{m+1}=0$ of $S$ as follows: let $b_{1}:=a_{1}$ and $\mathbf{H}^{1}:=\mathbf{C}_{\mathbf{G}}\left(\tilde{\gamma}_{b_{1}}\right)$. Let $b_{2}$ be the maximal element in $\left\{a_{2}, \cdots, a_{m+1}\right\}$ with the property that if $a_{j}<b_{2}, \tilde{\gamma}_{a_{j}} \in \mathfrak{z}_{\mathfrak{h}_{1}}$. Note that $\mathbf{H}^{1}=\mathbf{C}_{\mathbf{G}}\left(\tilde{\gamma}_{b_{1}, b_{2}}\right)$. Let $\mathbf{H}^{2}:=\mathbf{C}_{\mathbf{H}^{1}}\left(\tilde{\gamma}_{b_{2}}\right)$. Then $\mathbf{H}^{1} \supsetneq \mathbf{H}^{2}$. Let $\gamma_{b_{1}}:=\tilde{\gamma}_{b_{1}, b_{2}}$. Inductively, suppose $b_{i}, \mathbf{H}^{i}$ and $\gamma_{b_{i-1}}$ are defined for $i \geq 2$. Let $b_{i+1}$ be the maximal element in $\left\{a_{j} \in S \mid a_{j}>b_{i}\right\}$ with the property that for any $a_{j}<b_{i+1}, \tilde{\gamma}_{a_{j}} \in \mathfrak{z}_{\mathfrak{h}^{i}}$. Let $\mathbf{H}^{i+1}:=\mathbf{C}_{\mathbf{H}^{i}}\left(\tilde{\gamma}_{b_{i+1}}\right)$ and $\gamma_{b_{i}}=\tilde{\gamma}_{b_{i}, b_{i+1}}$. We repeat the process until $b_{n+1}=0$. Then each $\gamma_{b_{i}}$ is also a $G$-good element of depth $b_{i}$ by Lemma $4.6(2)$, and $\mathbf{H}^{i}=\mathbf{C}_{\mathbf{H}^{i-1}}\left(\tilde{\gamma}_{b_{i}}\right)=\mathbf{C}_{\mathbf{H}^{i-1}}\left(\gamma_{b_{i}}\right)$. Now, one can easily check

$$
\gamma=\gamma_{b_{1}}+\gamma_{b_{2}}+\cdots+\gamma_{b_{n}}+\gamma_{\circ}
$$

satisfies the required properties.

For the second statement, let $\gamma=\gamma_{b_{1}^{\prime}}^{\prime}+\gamma_{b_{2}^{\prime}}^{\prime}+\cdots+\gamma_{b_{n^{\prime}}^{\prime}}^{\prime}+\gamma_{\circ}^{\prime}$ be another expression satisfying (1)-(3). Then $b_{1}=\mathrm{d}(\gamma)=b_{1}^{\prime}$. Since $\gamma_{b_{1}} \equiv \gamma_{b_{1}^{\prime}}^{n^{\prime}}\left(\bmod \mathfrak{t}_{b_{1}^{+}}\right)$and $\gamma_{b_{1}}, \gamma_{b_{1}^{\prime}}^{\prime}$ are good, $\mathbf{H}^{1}=\mathbf{H}^{\prime 1}$ by Lemma 4.6. By induction, we assume that $b_{j}=b_{j}^{\prime}$ and $\mathbf{H}^{j}=\mathbf{H}^{j}$ for $1 \leq j \leq i-1$. Write $\gamma_{b_{1}, b_{i}}:=\gamma_{b_{1}}+\cdots+\gamma_{b_{i-1}}$ and $\gamma_{b_{1}, b_{i}^{\prime}}^{\prime}:=\gamma_{b_{1}}^{\prime}+\cdots+\gamma_{b_{i-1}}^{\prime}$. Suppose $b_{i}<b_{i}^{\prime}$. Then, $\gamma_{b_{1}, b_{i}^{\prime}}^{\prime}-\gamma_{b_{1}, b_{i}} \equiv \gamma_{b_{i}}\left(\bmod \mathfrak{t}_{b_{i}^{+}}\right)$. Since $\gamma_{b_{i}}$ is also $\mathbf{H}^{i-1}$-good, by Lemma 4.5 (2), we have $\mathbf{H}^{i-1} \subseteq \mathbf{H}^{i}$, which is a contradiction. Hence $b_{i}=b_{i}^{\prime}$. Now we have $(i) \gamma_{b_{i}} \equiv \gamma_{b_{1}, b_{i}}^{\prime}-\gamma_{b_{1}, b_{i}}+\gamma_{b_{i}}^{\prime}\left(\bmod \mathfrak{t}_{b_{i}^{+}}\right),(i i) \gamma_{b_{1}, b_{i}}^{\prime}-\gamma_{b_{1}, b_{i}} \in \mathfrak{z}_{\mathfrak{h}^{i-1}}$, and (iii) $\gamma_{b_{i}}, \gamma_{b_{1}, b_{i}}^{\prime}-\gamma_{b_{1}, b_{i}}+\gamma_{b_{i}}^{\prime}$ are good in $\mathbf{H}^{i-1}$ by 4.4 From these, it follows that $\mathbf{C}_{\mathbf{H}^{i-1}}\left(\gamma_{b_{i}}\right)=\mathbf{C}_{\mathbf{H}^{i-1}}\left(\gamma_{b_{i}}^{\prime}\right)$. Hence, $\mathbf{H}^{i}=\mathbf{H}^{\prime i}$.

Remark 4.8. By Lemma 4.5, we have $\mathbf{H}^{j}=\mathbf{C}_{\mathbf{G}}\left(\gamma_{b_{1}}+\cdots+\gamma_{b_{j}}\right)$ and $\mathbf{C}_{\mathbf{G}}(\gamma) \subset \mathbf{H}^{n}$.

\section{K-TYPES: BASIC DATA AND CONSTRUCTION}

In this section, we review the K-types constructed in [27. We will prove later that under some hypotheses, almost every irreducible tempered representation of positive depth contains one of these types (see 111). The construction is based on the following data:

Definition 5.1. A G-datum of positive depth is a quadruple $\Sigma=(\overrightarrow{\mathbf{G}}, y, \vec{r}, \vec{\phi})$ satisfying the following conditions (D1)-(D4). 
(D1) $\overrightarrow{\mathbf{G}}=\left(\mathbf{G}^{0} \subsetneq \mathbf{G}^{1} \subsetneq \cdots \subsetneq \mathbf{G}^{d}=\mathbf{G}\right)$ is a tamely ramified twisted Levi sequence. (D2) $y \in \mathcal{B}\left(\mathbf{G}^{0}, k\right)$.

(D3) $\vec{r}=\left(r_{0}, r_{1}, \cdots, r_{d-1}, r_{d}\right)$ is a sequence of positive real numbers with $0<r_{0}<$ $\cdots<r_{d-2}<r_{d-1} \leq r_{d}$.

(D4) $\vec{\phi}=\left(\phi_{0}, \cdots, \phi_{d}\right)$ is a sequence of quasi-characters, where $\phi_{i}$ is a quasicharacter of $G^{i}$. We assume that $\phi_{i}$ is trivial on $G_{y, r_{i}^{+}}^{i}$ but nontrivial on $G_{y, r_{i}}^{i}$ for $0 \leq i \leq d-1$. If $r_{d-1}<r_{d}$, we assume $\phi_{d}$ is nontrivial on $G_{y, r_{d}}^{d}$ and trivial on $G_{y, r_{d}^{+}}^{d}$. Otherwise, we assume that $\phi_{d}=1$.

We define the length $\ell(\Sigma)$ of the above $G$-datum of positive depth $\Sigma$ to be $d$.

Remark 5.2. As mentioned in the introduction, Yu defined a notion of $G$-datum for supercuspidal types (on which the above definition of $G$-datum of positive depth is based). However, the representations containing K-types constructed out of the above data are not necessarily supercuspidal.

Notation AND CONVENTIONS.

(1) Let $\mathbf{G}^{-1}:=\mathbf{G}^{0}, \mathfrak{g}^{-1}:=\mathfrak{g}^{0}$, and let $\mathbf{G}^{d+1}:=\mathbf{G}^{d}, \mathfrak{g}^{d+1}:=\mathfrak{g}^{d}$.

(2) Let $\mathbf{Z}_{\mathbf{G}^{i}}=\mathbf{Z}^{i}$ and $\mathfrak{z}_{\mathfrak{g}^{i}}=\mathfrak{z}^{i}$ denote the centers of $\mathbf{G}^{i}$ and $\mathfrak{g}^{i}$, respectively.

Definition 5.3. Let $\Sigma=(\overrightarrow{\mathbf{G}}, y, \vec{r}, \vec{\phi})$ be a $G$-datum of positive depth.

(1) We say $\Sigma$ is good if each $\phi_{i}, 0 \leq i \leq d-1$, is good and $\phi_{d}$ is either good or trivial. That is, for $0 \leq i \leq d-1, \phi_{i} \mid G_{y, r_{i}}^{i}$ is represented by a $G^{i+1}$-good element $\Gamma_{i} \in \mathfrak{z}^{i}$ of depth $-r_{i}$ such that $\mathbf{C}_{\mathbf{G}^{i+1}}\left(\Gamma_{i}\right)=\mathbf{G}^{i}$, and for $i=d$, either $\phi_{d}$ is trivial or $\phi_{d} \mid G_{y, r_{d}}^{d}$ is represented by $\Gamma_{d} \in \mathfrak{z}^{d}$ of depth $-r_{d}$.

(2) We say $\phi_{i}$ is strongly good if $\phi_{i} \mid G_{y, 0^{+}}^{i}$ is represented by a $G$-good element $\Gamma_{i} \in \mathfrak{z}^{i}$ of depth $-r_{i}$ such that $\mathbf{C}_{\mathbf{G}^{i+1}}\left(\Gamma_{i}\right)=\mathbf{G}^{i}$.

(3) We say $\Sigma$ is strongly good if each $\phi_{i}, 0 \leq i \leq d-1$, is strongly good and $\phi_{d}$ is either strongly good or trivial. Put $\Gamma_{d}=0$ if $\phi_{d}$ is trivial, and define $\Gamma_{\Sigma}$ (or simply $\Gamma$ ) and $\Gamma^{i}$ as follows:

$$
\begin{aligned}
& \Gamma^{i}:=\Gamma_{d}+\Gamma_{d-1}+\cdots+\Gamma_{i}, \\
& \Gamma_{\Sigma}=\Gamma:=\Gamma^{0}=\Gamma_{d}+\Gamma_{d-1}+\cdots+\Gamma_{0} .
\end{aligned}
$$

Remarks 5.4. Let $\Sigma$ be a $G$-datum of positive depth.

(1) The expression of $\Gamma_{\Sigma}$ as $\Gamma_{d}+\Gamma_{d-1}+\Gamma_{0}$ satisfies the condition in Proposition 4.7

(2) In the above definition, $\Gamma_{i}$ being $G$-good implies that $\Gamma_{i}$ is $G^{i+1}$-good. Hence, if $\Sigma$ is strongly good, it is also good. Note also that our definition of strongly good is stronger than the one in [27].

(3) If $\phi_{i}$ is good, it is also generic in the sense of [38. Hence we can apply most results in 38 to a (strongly) good $G$-datum of positive depth.

(4) Observe that each $\Gamma_{i} \in \mathfrak{z}_{\mathfrak{g}^{0}}$. Hence the $\Gamma_{i}$ 's commute with each other.

Lemma 5.5. Suppose $(\mathrm{HB})$ and $(\mathrm{H} k)$ are valid. Suppose $\gamma \in \mathfrak{z} \mathfrak{g}$ and $\mathrm{d}(\gamma)<$ 0 . Then there is a character $\phi$ of $G$ such that for any $x \in \mathcal{B}(\mathbf{G}, k), \phi \mid G_{x, 0^{+}}$is represented by $\gamma$.

Proof. Write $Z=Z_{G}$ for simplicity. Set $Z_{0^{+}}=\exp \left(\mathfrak{z}_{0^{+}}\right)$where $\mathfrak{z}_{0^{+}}=\mathfrak{z} \cap \mathfrak{t}_{0^{+}}$with $\mathbf{T}$ a maximal $k$-torus in $\mathbf{G}$. Since $Z$ is commutative, by $(\mathrm{H} k), \chi_{\gamma}$ defines a character of $Z_{0^{+}}$. Since the commutator $(G, G)$ is a subgroup of $G^{\text {der }}, G / G^{\text {der }}$ is abelian. 
Moreover, since we have an orthogonal decomposition $\mathfrak{g}_{x, 0^{+}}=\mathfrak{z}_{0^{+}} \oplus \mathfrak{g}_{x, 0^{+}}^{\text {der }}$ with respect to the bilinear form B by [4, (3.2)], we have $G_{x, 0^{+}}=\exp \left(\mathfrak{z}_{0^{+}} \oplus \mathfrak{g}_{x, 0^{+}}^{\mathrm{der}}\right)=$ $Z_{0^{+}} G_{x, 0^{+}}^{\text {der }}$ by $(\mathrm{H} k)$. Hence $Z_{0^{+}}$is embedded in $G / G^{\text {der }}$, and $\chi_{\gamma}$ defines a character $\bar{\chi}_{\gamma}$ of $Z_{0^{+}} G^{\text {der }} / G^{\text {der }}$. This character easily extends to a character of $Z G^{\text {der }} / G^{\text {der }}$, which we again denote by $\bar{\chi}_{\gamma}$. Now, since $G /\left(Z G^{\text {der }}\right)$ is finite, $\bar{\chi}_{\gamma}$ again extends to a character of $G / G^{\mathrm{der}}$, which induces a character $\phi$ of $G$.

It remains to show that $\phi \mid G_{x, 0^{+}}$is represented by $\gamma$. For $g \in G_{x, 0^{+}}$, we can write $g=\exp (z) \exp \left(z^{\prime}\right)=\exp \left(z+z^{\prime}\right)$ for some $z \in \mathfrak{z}_{0^{+}}$and $z^{\prime} \in \mathfrak{g}_{x, 0^{+}}^{\text {der }}$. Then, since $\phi$ is trivial on $G^{\text {der }}$ and $\mathfrak{g}=\mathfrak{z} \oplus \mathfrak{g}^{\text {der }}$ is an orthogonal decomposition with respect to $\mathrm{B}$, we have $\phi(g)=\phi(\exp (z))=\chi_{\gamma}(\exp (z))=\Lambda(\mathrm{B}(\gamma, z))=\Lambda\left(\mathrm{B}\left(\gamma, z+z^{\prime}\right)\right)=$ $\Lambda(\mathrm{B}(\gamma, \log (g)))$. Hence $\phi \mid G_{x, 0^{+}}$is represented by $\gamma$.

Remarks 5.6.

(1) Let $\phi$ be constructed as in the proof of Lemma 5.5. Then $\phi$ is trivial on $G^{\text {der }}$ and the depth of $\phi$ is $r=-\mathrm{d}(\gamma)$ independent of $x \in \mathcal{B}(\mathbf{G}, k)$. That is, for any $x \in \mathcal{B}(\mathbf{G}, k), \phi$ is trivial on $G_{x, r^{+}}$but nontrivial on $G_{x, r}$.

(2) Combining Lemma [5.5 and Proposition 4.7 for any semisimple element in $\mathfrak{g}$ of negative depth, one can associate a strongly good $G$-datum of positive depth.

Fix a good $G$-datum of positive depth $\Sigma=(\overrightarrow{\mathbf{G}}, y, \vec{r}, \vec{\phi})$. We review the construction of the K-type $\left(K_{\Sigma}^{+}, \phi_{\Sigma}\right)$ and some notation that we need in this paper.

5.7. Let the embeddings

$$
\mathcal{B}\left(\mathbf{G}^{0}, k\right) \hookrightarrow \mathcal{B}\left(\mathbf{G}^{1}, k\right) \hookrightarrow \mathcal{B}\left(\mathbf{G}^{2}, k\right) \hookrightarrow \cdots \hookrightarrow \mathcal{B}\left(\mathbf{G}^{d}, k\right)
$$

be fixed as in $₫ 2.3$. Let $s_{i}:=\frac{r_{i}}{2}$ for $i=0, \cdots, d-1$. For any $\epsilon \in \tilde{\mathbb{R}}$ with $0 \leq \epsilon<s_{0}$, define the following sequences $\vec{s}$ and $\vec{s}^{+}$of length $d$ :

$$
\vec{s}(\epsilon):=\left(\epsilon, s_{0}, \cdots, s_{d-1}\right), \quad \vec{s}^{+}(\epsilon):=\left(\epsilon, s_{0}^{+}, \cdots, s_{d-1}^{+}\right) .
$$

For simplicity, we write

$$
\vec{s}:=\vec{s}(0), \quad \vec{s}^{+}:=\vec{s}\left(0^{+}\right) .
$$

5.8. Define some open compact subgroups associated to $\Sigma$ as follows:

$$
\begin{array}{lll}
K_{\Sigma}^{i+} & :=G_{y, 0^{+}}^{0} G_{y, s_{0}^{+}}^{1} \cdots G_{y, s_{i-1}^{+}}^{i} & \subset G_{y, 0^{+}}^{i}, \\
K_{\Sigma+}^{i+1} & :=G_{y, s_{i}^{+}}^{i+1} \cdots G_{y, s_{d-1}^{+}}^{+} & \subset G_{y, s_{i}^{+}}^{d} \\
K_{\Sigma}^{+} & :=K_{\Sigma}^{i+} K_{\Sigma+}^{i+1}=G_{y, 0^{+}}^{0} G_{y, s_{0}^{+}}^{1} \cdots G_{y, s_{d-1}^{+}}^{d} & =\vec{G}_{y, \vec{s}^{+}} .
\end{array}
$$

Via the isomorphism $G_{y, s_{i}^{+}} / G_{y, r_{i}^{+}} \simeq \mathfrak{g}_{y, s_{i}^{+}} / \mathfrak{g}_{y, r_{i}^{+}}, \phi_{i}$ defines a character $\hat{\phi}_{i}$ of $G_{y, s_{i}^{+}}$ such that $\hat{\phi}_{i}$ is trivial on $\left(\mathfrak{g}_{y, s_{i}^{+}} \cap \mathfrak{g}_{\perp}^{i}\right) /\left(\mathfrak{g}_{y, r_{i}^{+}} \cap \mathfrak{g}_{\perp}^{i}\right)$ and $\hat{\phi}_{i}$ and $\phi_{i}$ coincide on $G_{y, s_{i}^{+}}^{i}\left(\right.$ see [38, §4]). Since $\phi_{i}$ is already defined on $K_{\Sigma}^{i+} \subset G_{y, 0^{+}}^{i}$, there is a unique character of $K_{\Sigma}^{+} G_{y, s_{i}^{+}}$extending $\phi_{i}$ and $\hat{\phi}_{i}$. We use the same notation $\hat{\phi}_{i}$ for this character of $K_{\Sigma}^{+} G_{y, s_{i}^{+}}$and its restriction to $K_{\Sigma}^{+}$. Now, define the character $\phi_{\Sigma}$ of $K_{\Sigma}^{+}$as $\prod_{i=0}^{d} \hat{\phi}_{i}$ :

$$
\phi_{\Sigma}:=\prod_{i=0}^{d} \hat{\phi}_{i} .
$$


5.9. Suppose $(\mathrm{H} k)$ is valid and $\Sigma$ is a strongly good $G$-datum of positive depth. Let $\Gamma_{i}$ and $\Gamma$ be as in Definition [5.3. We observe that $\hat{\phi}_{i}$ is represented by $\Gamma_{i}$ on $G_{y, 0^{+}}^{i} G_{y, s_{i}^{+}}$and hence on $K_{\Sigma}^{+}$, that is, $\hat{\phi}_{i}=\chi_{\Gamma_{i}}$ on $G_{y, 0^{+}}^{i} G_{y, s_{i}^{+}}$and on $K_{\Sigma}^{+}$. Moreover, we have

$$
\phi_{\Sigma}=\chi_{\Gamma}
$$

on $K_{\Sigma}^{+}$, and the dual blob of $\phi_{\Sigma}=\chi_{\Gamma}$ is $\Gamma+\left(\overrightarrow{\mathfrak{g}}_{y, \vec{s}^{+}}\right)^{*}=\Gamma+\overrightarrow{\mathfrak{g}}_{y,-\vec{s}}$, where $-\vec{s}=$ $\left(-s_{-1},-s_{0}, \cdots,-s_{d-1}\right)$. We also observe that

$$
\Gamma^{i}:=\Gamma_{d}+\Gamma_{d-1}+\cdots+\Gamma_{i}
$$

defines a character $\chi_{\Gamma^{i}}$ of $K_{\Sigma+}^{i+1}$. We also put $\Gamma^{-1}:=\Gamma^{0}=\Gamma$.

Remarks 5.10. Let $\Sigma=(\overrightarrow{\mathbf{G}}, y, \vec{r}, \vec{\phi})$ be a strongly good $G$-datum of positive depth.

(1) Since the construction of $\left(K_{\Sigma}^{+}, \phi_{\Sigma}\right)$ depends only on $\Gamma_{i}$ representing $\phi_{i}$ on $G_{y, 0^{+}}^{i}$, replacing $\phi_{i}$ with the characters constructed in Lemma 5.5 with $\gamma=\Gamma_{i}$ produces the same open compact subgroup and its representation. Hence, without loss of generality, we may and will assume that for a strongly good $G$-datum of positive depth $\Sigma$, each $\phi_{i}$ is represented by $\Gamma_{i}$ on $G_{x, 0^{+}}^{i}$ for any $x \in \mathcal{B}\left(\mathbf{G}^{i}, k\right)$. In this case, for any $x \in \mathcal{B}\left(\mathbf{G}^{0}, k\right),(\overrightarrow{\mathbf{G}}, x, \vec{r}, \vec{\phi})$ is also a strongly good $G$-datum of positive depth. We will often denote $(\overrightarrow{\mathbf{G}}, x, \vec{r}, \vec{\phi})$ by $\Sigma_{x}$.

(2) Since $\Gamma$ determines $\overrightarrow{\mathbf{G}}, \vec{r}$ and $\phi_{\Sigma}, \Sigma$ can be replaced by $(\Gamma, y)$ and they yield the same K-type $\left(K_{\Sigma}^{+}=\vec{G}_{y, \vec{s}^{+}}, \chi_{\Gamma}\right)$.

Remarks 5.11 (Some Properties of $\left(K_{\Sigma}^{+}, \phi_{\Sigma}\right)$ ).

(1) If an irreducible admissible representation $\left(\pi, V_{\pi}\right)$ contains $\left(K_{\Sigma}^{+}, \chi_{\Gamma}\right)$, the depth $\varrho(\pi)$ of $\pi$ is $r_{d}$.

(2) Let $\Sigma=(\overrightarrow{\mathbf{G}}, y, \vec{r}, \vec{\phi})$ be a good $G$-datum of positive depth. Suppose $\mathbf{Z}_{\mathbf{G}^{0}} / \mathbf{Z}_{\mathbf{G}}$ is anisotropic. Let $\rho$ be an irreducible representation of $G_{[y]}^{0}$, the stabilizer in $G^{0}$ of the image $[y]$ of $y$ in the reduced building of $\mathbf{G}^{0}$, such that $c-\operatorname{Ind}_{G_{[y]}^{0}}^{G^{0}} \rho$ is irreducible supercuspidal. Then $(\overrightarrow{\mathbf{G}}, y, \vec{r}, \vec{\phi}, \rho)$ is a generic $G$-datum ([38]; see also 12). Moreover, if $\pi$ is a supercuspidal representation constructed from the above generic datum, $\phi_{\Sigma}$ occurs in the restriction $\pi \mid K_{\Sigma}^{+}$of $\pi$ to $K_{\Sigma}^{+}$.

For more details and properties of the above K-types, we refer to [27].

5.12. Let $\Sigma=(\overrightarrow{\mathbf{G}}, y, \vec{r}, \vec{\phi})$ be as before. We recall some lattices and open compact subgroups associated to $\Sigma$ from [27].

(1) Let $\epsilon \in \mathbb{R}$ be such that $\mathfrak{g}_{\epsilon}=\mathfrak{g}_{0^{+}}$. For $x \in \mathcal{B}\left(\mathbf{G}^{0}, k\right)$, let

$$
\mathcal{L}_{x, \epsilon}=\mathcal{L}_{x}:=\overrightarrow{\mathfrak{g}}_{x, \vec{s}^{+}(\epsilon)}, \quad \mathcal{L}_{x, \epsilon}^{\sharp}=\mathcal{L}_{x}^{\sharp}:=\overrightarrow{\mathfrak{g}}_{x,\left(\vec{s}^{+}(\epsilon)\right)^{*}},
$$

and

$$
L_{x, \epsilon}=L_{x}:=\vec{G}_{x, \vec{s}^{+}(\epsilon)} .
$$

Although these definitions $\mathcal{L}_{x, \epsilon}, L_{x, \epsilon}$, etc., depend on $\overrightarrow{\mathbf{G}}, \vec{r}$ and the embedding of buildings, since we would not need their roles explicitly, we omit them from the subscripts for simplicity. If (HB) is valid, $\mathcal{L}_{x, \epsilon}^{\sharp}$ is just $\mathcal{L}_{x, \epsilon}^{*}$. If there is no confusion, we will drop $\epsilon$ from the notation, that is, we will write $\mathcal{L}_{x}, \mathcal{L}_{x}^{\sharp}, L_{x}$ for $\mathcal{L}_{x, \epsilon}, \mathcal{L}_{x, \epsilon}^{\sharp}, L_{x, \epsilon}$. 
(2) Assume (HB) is valid. For $i=1, \cdots, d+1$ and $x \in \mathcal{B}\left(\mathbf{G}^{i}, k\right)$, define $\mathcal{L}_{x}^{i}$ and $\mathcal{L}_{x}^{i \sharp}$ as follows:

$$
\begin{aligned}
& \mathcal{L}_{x}^{d+1}=\mathcal{L}_{x}^{d}:=\mathfrak{g}_{x, s_{d-1}^{+}}^{d}, \\
& \mathcal{L}_{x}^{i} \quad:=\mathfrak{g}_{x, s_{i-1}^{+}}^{i}+\mathcal{L}_{x}^{i+1} \quad \text { for } \quad i=1, \cdots, d-1, \\
& \mathcal{L}_{x}^{i \sharp} \quad:=\mathcal{L}_{x}^{i *}, \quad \text { for } i=1, \cdots, d+1 .
\end{aligned}
$$

Set $\mathcal{L}_{x}^{0}:=\mathcal{L}_{x, \epsilon}, \mathcal{L}_{x}^{0 \sharp}:=\mathcal{L}_{x, \epsilon}^{\sharp}$ and $L_{x}^{0}:=L_{x, \epsilon}$.

For later use, we record the following, which is a corollary of [27, (5.3.2)]:

Lemma 5.13. Assume the hypothesis $(\mathrm{H} k)$ is valid. Let $x \in \mathcal{B}\left(\mathbf{G}^{0}, k\right)$ and $X \in$ $\mathfrak{g}_{x,\left(-r_{0}\right)^{+}}^{0}$ Then

$$
\Gamma+X+\mathcal{L}_{x}^{\sharp} \subset{ }^{G}\left(\Gamma+X+\mathfrak{g}_{0}^{0}\right) .
$$

For our purpose, we also define a zero good $G$-datum.

Definition 5.14. A zero $G$-datum (or 0 -datum) $\Sigma$ is a $G$-datum of the form $\Sigma=$ $(\overrightarrow{\mathbf{G}}, y, \vec{r}, \vec{\phi})$ where $d=0, \overrightarrow{\mathbf{G}}=\left(\mathbf{G}^{0}=\mathbf{G}\right), \vec{r}=(0), \vec{\phi}=(1)$ and $y \in \mathcal{B}(\mathbf{G}, k)$. Then we associate the corresponding $\mathrm{K}$-types and lattices as follows:

$$
K_{\Sigma}^{+}=G_{y, 0^{+}}, \quad \phi_{\Sigma}=1, \quad \Gamma_{\Sigma}=\Gamma=0,
$$

and for $x \in \mathcal{B}(\mathbf{G}, k)$, independent of the choice of $\epsilon$,

$$
\mathcal{L}_{x}=\mathfrak{g}_{x, 0^{+}}, \quad \mathcal{L}_{x}^{\sharp}=\mathfrak{g}_{x, 0} .
$$

We associate real numbers $r_{0}, r_{-1}, s_{-1}$ and $s_{0}$ as a zero datum as follows:

$$
r_{0}=r_{-1}=s_{-1}=s_{0}=0 .
$$

Definition and Remark 5.15. Let $\Sigma$ be a zero datum. For an irreducible admissible representation $\left(\pi, V_{\pi}\right)$, if $\left(K_{\Sigma}^{+}, \phi_{\Sigma}\right)<\pi, \pi$ is a depth zero representation. By convention, we will call a zero datum both good and strongly good. Hence, by a (strongly) good $G$-datum, we mean either a zero or a (strongly) good $G$-datum of positive depth.

Definition and Remark 5.16.

(1) Let $\mathcal{E}$ denote the set of all irreducible admissible representations. Let $\mathcal{E}^{u}$ (resp. $\mathcal{E}^{t}$ ) denote the subset of $\mathcal{E}$ which consists of unitarizable (resp. tempered) representations of $G$.

We remark that $\mathcal{E}$ carries a natural topology defined via approximation of matrix cofficients. Moreover, $\mathcal{E}^{u}$ is a closed subset of $\mathcal{E}$, and the subspace topology on $\mathcal{E}^{u}$ coincides with the usual topology on the unitary dual. See [35] for more details.

(2) Let $J$ be an open compact subgroup of $G$ and $\sigma$ one of its irreducible representations. For $\left(\pi, V_{\pi}\right) \in \mathcal{E}$, let $V_{\pi}^{(J, \sigma)}$ (or simply $V_{\pi}^{\sigma}$ ) denote the $\sigma$-isotypic component in $V_{\pi}$. If $V_{\pi}^{\sigma} \neq 0$, that is, if $\sigma$ occurs as a subrepresentation of the restriction of $\pi$ to $J$, we write $(J, \sigma)<\pi$. 


\section{Review on Plancherel formulas on $G$ and $\mathfrak{g}$}

For $\left(\pi, V_{\pi}\right) \in \mathcal{E}^{u}$ and $F \in C_{c}^{\infty}(G)$, we define a function $\widehat{F}$ on $\mathcal{E}^{u}$ as

$$
\widehat{F}(\pi):=\operatorname{Tr}(\pi(F))=\Theta_{\pi}(F) .
$$

Then the Plancherel formula on locally compact groups (see [13]) states that there is a Borel measure $d \pi$ called Plancherel measure on $\mathcal{E}^{u}$ such that the first equality in the following holds:

$$
F(1)=\int_{\mathcal{E}^{u}} \widehat{F}(\pi) d \pi=\int_{\mathcal{E}^{t}} \widehat{F}(\pi) d \pi .
$$

From Harish-Chandra's explicit Plancherel formula ([17, 37]), we have the second equality.

On the other hand, regarding $\mathfrak{g}$ as a topological group with respect to addition, we can formulate the Plancherel formula on $\mathfrak{g}$ as follows: there is a Borel measure on $\widehat{\mathfrak{g}}$ such that for $f \in C_{c}^{\infty}(\widehat{\mathfrak{g}})$,

$$
f(0)=\int_{\widehat{\mathfrak{g}}} \widehat{f}(\chi) d \chi
$$

where $\widehat{f} \in C_{c}^{\infty}(\widehat{\mathfrak{g}})$ is the Fourier transformation of $f$ given by

$$
\widehat{f}(\chi)=\int_{\widehat{\mathfrak{g}}} f(Y) \chi(Y) d Y .
$$

Recall that we have the following isomorphisms:

$$
\widehat{\mathfrak{g}} \simeq \mathfrak{g}^{*} \simeq \mathfrak{g},
$$

where $\widehat{\mathfrak{g}}$ denotes the unitary dual of $\mathfrak{g}$. The first isomorphism is from Pontrjagin duality. We have the second isomorphism via an additive character and an appropriate bilinear form on $\mathfrak{g}$. When (HB) holds, we have $\widehat{\mathfrak{g}} \simeq \mathfrak{g}$ via $\Lambda$ and $\mathrm{B}$, and we can rewrite the above formula as

$$
f(0)=\int_{\mathfrak{g}} \widehat{f}(X) d X
$$

where $\widehat{f}(X)=\int_{\mathfrak{g}} f(Y) \Lambda(\mathrm{B}(X, Y)) d Y$. In the above equation (6.2), the $G$-invariant measure $d X$ on $\mathfrak{g}$ should satisfy $\operatorname{vol}_{\mathfrak{g}}\left(\mathfrak{g}_{x, r}\right) \operatorname{vol}_{\mathfrak{g}}\left(\mathfrak{g}_{x, r^{*}}\right)=1$ for all $x \in \mathcal{B}(\mathbf{G}, k)$ and $r \in \mathbb{R}$.

To relate Plancherel formulas on $\mathfrak{g}$ and $G$, let $f \in C_{c}^{\infty}(\mathfrak{g})$ be supported in a sufficiently small neighborhood of 0 . Then $f \circ \log$ defines a function in $C_{c}^{\infty}(G)$. Combining the Plancherel formulas (6.1) and (6.2), we have

$$
\int_{\mathfrak{g}} \widehat{f}(X) d X=f(0)=\int_{\mathcal{E}^{t}} \Theta_{\pi}(f \circ \log ) d \pi .
$$

6.1. Haar measures. From now on, when (HB) is valid, we fix a Haar measure on $\mathfrak{g}$ so that (6.2) is valid. When $(\mathrm{H} k)$ is valid, we fix a Haar measure on $G$ so that $\operatorname{vol}_{G}\left(G_{x, f}\right)=\operatorname{vol}_{\mathfrak{g}}\left(\mathfrak{g}_{x, f}\right)$ for any $x$ and $f$ as in $(\mathrm{H} k)$. Then, the Plancherel measure $d \pi$ in (6.1) is uniquely determined with respect to this Haar measure on $G$. 


\section{Decomposition of $\mathfrak{g}$}

Throughout this section, we assume that (HGT) is valid. The main result in this section is Proposition 7.6, where we find a spectral decomposition of (6.2). Each spectral decomposition factor is parameterized by an equivalence class of semisimple elements (see Definition 7.4).

7.1. We restate Proposition 4.7 to fit better for our purpose: let $\Gamma \in \mathfrak{g}$ be a semisimple element. Then $\Gamma$ can be written as

$$
\Gamma=\Gamma_{d}+\Gamma_{d-1}+\cdots+\Gamma_{0}+\gamma
$$

satisfying the following:

(1) $\Gamma_{i}, i=0,1, \cdots, d-1$, is a good element of depth $-r_{i}$ with $-r_{d-1}<-r_{d-2}<$ $\cdots<-r_{0}<0$, and $\mathrm{d}(\gamma) \geq 0$.

(2) $\Gamma_{d}=0$ or $\Gamma_{d} \in \mathfrak{z}^{d}=\mathfrak{z}_{\mathfrak{g}}$ is nonzero with $\mathrm{d}\left(\Gamma_{d}\right)<-r_{d-1}$.

(3) $\mathbf{G}^{0} \subsetneq \mathbf{G}^{1} \subsetneq \cdots \subsetneq \mathbf{G}^{d}=\mathbf{G}$ where $\mathbf{G}^{i}=\mathbf{C}_{\mathbf{G}^{i+1}}\left(\Gamma_{i}\right)=\mathbf{C}_{\mathbf{G}}\left(\Gamma^{i}\right)$ with $\Gamma^{i}=\Gamma_{d}+\cdots+\Gamma_{i-1}+\Gamma_{i}$.

Let $r_{d}:=r_{d-1}$ if $\Gamma_{d}=0$, and let $r_{d}:=-\mathrm{d}\left(\Gamma_{d}\right)$ if $\Gamma_{d} \neq 0$. Then, $\vec{r}:=\left(r_{0}, \cdots, r_{d}\right)$ and $\overrightarrow{\mathbf{G}}:=\left(\mathbf{G}^{0}, \mathbf{G}^{1}, \cdots, \mathbf{G}^{d}\right)$ are determined uniquely independent of the choice of good elements $\Gamma_{i}$. Note that $\mathrm{d}(\Gamma)=-r_{d}$.

Definition and Remark 7.2. We keep the notation from \$7.1. Write $\Gamma=\Gamma^{0}+\gamma$ where $\Gamma^{0}=\Gamma_{d}+\Gamma_{d-1}+\cdots+\Gamma_{0}$. Define $\mathfrak{g}_{\Gamma}^{0}$ as follows:

$$
\mathfrak{g}_{\Gamma}^{0}:=\mathfrak{g}_{0}^{0}=\bigcup_{x \in \mathcal{B}\left(\mathbf{G}^{0}, k\right)} \mathfrak{g}_{x, 0}^{0} .
$$

Since $\mathbf{G}^{0}=\mathbf{C}_{\mathbf{G}}\left(\Gamma^{0}\right)$ is well defined independent of the choice of $\Gamma^{0}$, so is $\mathfrak{g}_{\Gamma}^{0}$.

Lemma 7.3. Let $\Gamma$ and $\Gamma^{\prime}$ be two semisimple elements in $\mathfrak{g}$.

(1) ${ }^{G}\left(\Gamma^{0}+\mathfrak{g}_{\Gamma}^{0}\right)$ is a $G$-domain, that is, an open and closed $G$-invariant subset of $\mathfrak{g}$.

(2) ${ }^{G}\left(\Gamma^{0}+\mathfrak{g}_{\Gamma}^{0}\right)$ and ${ }^{G}\left(\Gamma^{\prime 0}+\mathfrak{g}_{\Gamma^{\prime}}^{0}\right)$ are either disjoint or identical.

Proof. (1) follows from [27, (5.1.4)]. To prove (2), suppose

$$
{ }^{G}\left(\Gamma^{0}+\mathfrak{g}_{\Gamma}^{0}\right) \cap{ }^{G}\left(\Gamma^{\prime 0}+\mathfrak{g}_{\Gamma^{\prime}}^{0}\right) \neq \emptyset .
$$

Without loss of generality, we may assume $\left(\Gamma^{0}+\mathfrak{g}_{\Gamma}^{0}\right) \cap\left(\Gamma^{\prime 0}+\mathfrak{g}_{\Gamma^{\prime}}^{0}\right) \neq \emptyset$. Then there are $X \in \mathfrak{g}_{\Gamma}^{0}$ and $X^{\prime} \in \mathfrak{g}_{\Gamma^{\prime}}^{0}$ such that $Y:=\Gamma^{0}+X=\Gamma^{\prime 0}+X^{\prime}$. Let $X=X_{s}+X_{n}$ be the Jordan decomposition of $X$ in $\mathfrak{c}_{\mathfrak{g}}\left(\Gamma^{0}\right)$. Similary, write $X^{\prime}=X_{s}^{\prime}+X_{n}^{\prime}$. Then, $\left(\Gamma^{0}+X_{s}\right)+X_{n}$ and $\left(\Gamma^{\prime 0}+X_{s}^{\prime}\right)+X_{n}^{\prime}$ are two expressions of the Jordan decomposition of $Y$. By the uniqueness of Jordan decomposition, we have $\Gamma^{\prime \prime}:=\Gamma^{0}+X_{s}=\Gamma^{\prime 0}+X_{s}^{\prime}$. Note that $\mathrm{d}\left(X_{s}\right)=\mathrm{d}(X) \geq 0$ and $\mathrm{d}\left(X_{s}^{\prime}\right)=\mathrm{d}\left(X^{\prime}\right) \geq 0$. Applying Proposition 4.7 to $\Gamma^{\prime \prime}$, we conclude $C_{G}\left(\Gamma^{\prime \prime 0}\right)=C_{G}\left(\Gamma^{0}\right)=C_{G}\left(\Gamma^{\prime 0}\right)$. Hence, $\mathfrak{g}_{\Gamma^{\prime \prime}}^{0}=\mathfrak{g}_{\Gamma}^{0}=\mathfrak{g}_{\Gamma^{\prime}}^{0}$, $\left(\Gamma^{\prime \prime}+\mathfrak{g}_{\Gamma^{\prime \prime}}^{0}\right)=\left(\Gamma^{0}+\mathfrak{g}_{\Gamma}^{0}\right)=\left(\Gamma^{\prime 0}+\mathfrak{g}_{\Gamma^{\prime}}^{0}\right)$, and thus ${ }^{G}\left(\Gamma^{0}+\mathfrak{g}_{\Gamma}^{0}\right)={ }^{G}\left(\Gamma^{\prime 0}+\mathfrak{g}_{\Gamma^{\prime}}^{0}\right)$.

Definition 7.4. Let $\Gamma$ and $\Gamma^{\prime}$ be semisimple elements in $\mathfrak{g}$.

(1) Define $\mathfrak{g}_{\Gamma}:={ }^{G}\left(\Gamma^{0}+\mathfrak{g}_{\Gamma}^{0}\right)$.

(2) We say that $\Gamma$ and $\Gamma^{\prime}$ are equivalent if $\mathfrak{g}_{\Gamma}=\mathfrak{g}_{\Gamma^{\prime}}$. In that case, we write $\Gamma \sim \Gamma^{\prime}$. Let $\mathfrak{S}$ be the set of equivalence classes of semisimple elements.

(3) If $\Gamma \sim 0$, we set $d=0$ and the associated real numbers $r_{0}=r_{-1}=s_{0}=$ $s_{-1}=0$. 
By Lemma 7.3 the above equivalence relation is well defined.

Examples. (1) Any two semisimple elements $\Gamma$ and $\Gamma^{\prime}$ with $\mathrm{d}(\Gamma) \geq 0$ and $\mathrm{d}\left(\Gamma^{\prime}\right) \geq 0$ are equivalent. In this case, we have $\mathfrak{g}_{\Gamma}=\mathfrak{g}_{\Gamma^{\prime}}=\mathfrak{g}_{0}$.

(2) Let $\mathfrak{t}$ be a $k$-torus in $\mathfrak{g}$ which splits over a tamely ramified extension. Any $\Gamma, \Gamma^{\prime} \in \mathfrak{t}$ satisfying $\Gamma \equiv \Gamma^{\prime}\left(\bmod \mathfrak{t}_{0}\right)$ are equivalent.

Lemma 7.5. The Lie algebra $\mathfrak{g}$ is a disjoint union of $\mathfrak{g}_{\Gamma}={ }^{G}\left(\Gamma^{0}+\mathfrak{g}_{\Gamma}^{0}\right), \Gamma \in \mathfrak{S}$ :

$$
\mathfrak{g}=\bigcup_{\Gamma \in \mathfrak{S}}{ }^{G}\left(\Gamma^{0}+\mathfrak{g}_{\Gamma}^{0}\right)=\bigcup_{\Gamma \in \mathfrak{S}^{\circ}} \mathfrak{g}_{\Gamma} .
$$

Proof. ' $\supset$ ' is obvious. For ' $\subset$ ', let $X \in \mathfrak{g}$. Let $X=X_{s}+X_{n}$ be the Jordan decomposition of $X$ and $G^{\prime}:=C_{G}\left(X_{s}\right)$. Since $X_{n}$ is nilpotent and $X_{n} \in \mathfrak{g}^{\prime}$, we have $X_{n} \in \mathfrak{g}_{x, 0}^{\prime}$ for some $x \in \mathcal{B}\left(G^{\prime}\right)$. Hence $X \in \mathfrak{g}_{X_{s}}$, and ' $\subset$ ' follows.

Proposition 7.6. For any $f \in C_{c}^{\infty}(\mathfrak{g})$, the integral $\int_{\mathfrak{g}} f(X) d X$ is decomposed as follows:

$$
\int_{\mathfrak{g}} f(X) d X=\sum_{\Gamma \in \mathfrak{S}} \int_{\mathfrak{g}_{\Gamma}} f(X) d X .
$$

Proof. This follows from Lemma 7.3(1) and Lemma 7.5.

Corollary 7.7. Suppose (HB) is valid. Then, for any $f \in C_{c}^{\infty}(\mathfrak{g})$,

$$
f(0)=\int_{\mathfrak{g}} \widehat{f}(X) d X=\sum_{\Gamma \in \mathfrak{S}} \int_{\mathfrak{g}_{\Gamma}} \widehat{f}(X) d X .
$$

\section{Decomposition of $\mathcal{E}$}

8.1. From now on, we fix $\epsilon$ such that $\mathfrak{g}_{\epsilon}=\mathfrak{g}_{0^{+}}$. Since $\left\{r \in \mathbb{R} \mid \mathfrak{g}_{r} \supsetneq \mathfrak{g}_{r^{+}}\right\}$is a discrete subset of $\mathbb{R}$, such an $\epsilon$ exists.

The choice of such an $\epsilon$ first appears in [11] to treat homogeneity in the depth zero case. This setting is carried to [27. Via this choice, we can find nice test functions which are in a certain sense dual to the orbital integrals in which we are interested (see [27, (9.1.6)] or \$9.5).

8.2. Recall that for each twisted Levi sequence $\overrightarrow{\mathbf{G}}$, we fixed embeddings of buildings in 42.3 Except when $\mathbf{Z}^{0} / \mathbf{Z}^{d}$ is $k$-anisotropic, most objects defined here might in principle depend on the K-types constructed in $\S 5$, hence on the choice of embeddings. In the course of the proof of our main result, we show that if $\overrightarrow{\mathbf{G}}$ is associated to a supercuspidal representation, then $\mathbf{Z}^{0} / \mathbf{Z}^{d}$ is $k$-anisotropic (Proposition 14.5). Hence, the ambiguity in the choice of embedding is irrelevant for the particular argument pursued in this paper.

Throughout this section, we assume (Hk), (HB) and (HGT) are valid.

The main result in this section is Proposition 8.11, where we find a spectral decomposition of (6.1). In $\S 11$, we will see that each decomposition factor is parameterized by an element of $\mathfrak{S}$.

Definition 8.3. Let $\Sigma=(\overrightarrow{\mathbf{G}}, y, \vec{r}, \vec{\phi})$ be a strongly good $G$-datum of positive depth, and let $\Gamma_{\Sigma}$ be a semisimple element associated to $\Sigma$ as in Definition 5.3 . Let $\Gamma$ be a semisimple element in $\mathfrak{g}$.

(1) We say that $\Sigma$ and $\Gamma$ are associated if $\Gamma_{\Sigma} \sim \Gamma$. 
(2) $\mathcal{E}_{\Sigma}(\epsilon):=\left\{\left(\pi, V_{\pi}\right) \in \mathcal{E} \mid \pi>\left(L_{x, \epsilon}, \chi_{\Gamma_{\Sigma}+n}\right)\right.$, for some $x \in \mathcal{B}\left(\mathbf{G}^{0}, k\right), n \in$ $\left.\mathfrak{g}_{x,-\epsilon}^{0} \cap \mathcal{N}\right\}$.

Remarks 8.4.

(1) Note that any two semisimple elements associated to $\Sigma$ as in Definition 5.3 are equivalent. Hence, (1) is well defined.

(2) The definition in (2) is set up to achieve the equality in Theorem 10.1. Note that $n \in \mathfrak{g}_{x,-\epsilon}^{0}$ defines a character on $G_{x, \epsilon}$ and $L_{x, \epsilon}$ because $G_{x, \epsilon} / G_{x, \epsilon^{+}}$is abelian. Hence, $\Gamma_{\Sigma}+n$ also defines a character on $L_{x, \epsilon}$.

Definition 8.5. Let $\Gamma$ be a semisimple element in $\mathfrak{g}$.

(1) If $\mathrm{d}(\Gamma)<0$,

$$
\mathcal{E}_{\Gamma}(\epsilon):=\bigcup_{\Gamma_{\Sigma} \sim \Gamma} \mathcal{E}_{\Sigma}(\epsilon)
$$

where the union runs over all strongly good $G$-datum $\Sigma$ of positive depth such that $\Gamma_{\Sigma} \sim \Gamma$.

(2) If $\Gamma \sim 0$, define $\mathcal{E}_{0}=\mathcal{E}_{\Gamma}(\epsilon)$ to be the set of all depth zero representations (this is independent of the choice of $\epsilon$ ).

Lemma 8.6. Let $\Gamma$ and $\Gamma^{\prime}$ be semisimple elements in $\mathfrak{g}$.

(1) $\varepsilon_{\Gamma}(\epsilon)=\varepsilon_{\Gamma^{\prime}}(\epsilon)$ if and only if $\Gamma \sim \Gamma^{\prime}$.

(2) $\mathcal{E}_{\Gamma}(\epsilon)$ and $\mathcal{E}_{\Gamma^{\prime}}(\epsilon)$ are either disjoint or identical.

Proof. (1) ' $\Leftarrow$ ' is obvious. For ' $\Rightarrow$ ', if $\mathcal{E}_{\Gamma}(\epsilon) \cap \mathcal{E}_{\Gamma^{\prime}}(\epsilon) \neq \emptyset$, then there is a strongly good $G$-datum of positive depth $\Sigma$ such that $\Gamma \sim \Gamma_{\Sigma} \sim \Gamma^{\prime}$. Hence $\Gamma \sim \Gamma^{\prime}$.

(2) Note that the depth of a representation in $\mathcal{E}_{\Gamma}(\epsilon)$ is either 0 or $-\mathrm{d}(\Gamma)$. Suppose $\Gamma \sim 0$. Then, $\mathcal{E}_{\Gamma}(\epsilon)=\mathcal{E}_{0}$, and $\mathcal{E}_{\Gamma^{\prime}}(\epsilon)$ intersects $\mathcal{E}_{0}$ if and only if $\Gamma^{\prime} \sim 0$. Hence, $\mathcal{E}_{\Gamma^{\prime}}(\epsilon)=\mathcal{E}_{\Gamma}(\epsilon)$.

Now, suppose $\mathrm{d}(\Gamma), \mathrm{d}\left(\Gamma^{\prime}\right)<0$. Suppose $\left(\pi, V_{\pi}\right) \in \mathcal{E}_{\Gamma}(\epsilon) \cap \mathcal{E}_{\Gamma^{\prime}}(\epsilon)$. Then there are two strongly good $G$-datums of positive depth $\Sigma=(\overrightarrow{\mathbf{G}}, y, \vec{r}, \vec{\phi})$ and $\Sigma^{\prime}=\left(\overrightarrow{\mathbf{G}}^{\prime}, y^{\prime}, \vec{r}^{\prime}, \vec{\phi}^{\prime}\right)$ such that

(i) $\Gamma_{\Sigma} \sim \Gamma$ and $\left(\pi, V_{\pi}\right)>\left(L_{x, \epsilon}, \chi_{\Gamma_{\Sigma}+n}\right)$ for some $x \in \mathcal{B}\left(\mathbf{G}^{0}, k\right)$ and $n \in \mathfrak{g}_{x,-\epsilon}^{0}$,

(ii) $\Gamma_{\Sigma^{\prime}} \sim \Gamma^{\prime}$ and $\left(\pi, V_{\pi}\right)>\left(L_{x^{\prime}, \epsilon}, \chi_{\Gamma_{\Sigma^{\prime}}+n^{\prime}}\right)$ for some $x^{\prime} \in \mathcal{B}\left(\mathbf{G}^{\prime 0}, k\right)$ and $n^{\prime} \in$ $\mathfrak{g}_{x^{\prime},-\epsilon}^{\prime 0}$.

Since $\left(\pi, V_{\pi}\right)$ is irreducible, an argument similar to the one in [30, (7.2)] shows that there is $g \in G$ such that ${ }^{g} \chi_{\Gamma_{\Sigma}+n}=\chi_{\Gamma_{\Sigma^{\prime}}+n^{\prime}}$ on ${ }^{g} L_{x, \epsilon} \cap L_{x^{\prime}, \epsilon}^{\prime}$. Then, by Lemma 3.12, $g\left(\Gamma_{\Sigma}+n+\mathcal{L}_{x, \epsilon}^{\sharp}\right) \cap\left(\Gamma_{\Sigma^{\prime}}+n^{\prime}+\mathcal{L}_{x^{\prime}, \epsilon}^{\sharp \sharp}\right) \neq \emptyset$. Since $n \in \mathfrak{g}_{\Gamma_{\Sigma}}^{0}$ and $n^{\prime} \in \mathfrak{g}_{\Gamma_{\Sigma^{\prime}}}^{0}$, by Lemma 5.13, $\Gamma_{\Sigma}+n+\mathcal{L}_{x, \epsilon}^{\sharp} \subset{ }^{G}\left(\Gamma_{\Sigma}+\mathfrak{g}_{\Gamma_{\Sigma}}^{0}\right)$ and $\Gamma_{\Sigma^{\prime}}+n^{\prime}+\mathcal{L}_{x, \epsilon}^{\sharp} \subset{ }^{G}\left(\Gamma_{\Sigma^{\prime}}+\mathfrak{g}_{\Gamma_{\Sigma^{\prime}}}^{0}\right)$. Hence, ${ }^{G}\left(\Gamma_{\Sigma}+\mathfrak{g}_{\Gamma_{\Sigma}}^{0}\right) \cap{ }^{G}\left(\Gamma_{\Sigma^{\prime}}+\mathfrak{g}_{\Gamma_{\Sigma^{\prime}}}^{0}\right) \neq \emptyset$, and $\Gamma_{\Sigma} \sim \Gamma_{\Sigma^{\prime}}$ by Lemma $7.3(2)$. Now, we have $\mathcal{E}_{\Gamma}(\epsilon)=\mathcal{E}_{\Gamma^{\prime}}(\epsilon)$ by $(1)$.

\section{Definition 8.7.}

(1) Let $\mathcal{E}_{\Gamma}^{t}(\epsilon):=\mathcal{E}^{t} \cap \mathcal{E}_{\Gamma}(\epsilon)$.

(2) For any pair $\mathfrak{s}=(J, \sigma)$ consisting of an open compact subgroup $J$ and its irreducible representation $\sigma$, we define subsets $\mathcal{E}_{\mathfrak{s}}$ and $\mathcal{E}_{\mathfrak{s}}^{t}$ of $\mathcal{E}$ as follows:

$$
\mathcal{E}_{\mathfrak{s}}:=\{\pi \in \mathcal{E} \mid \mathfrak{s}<\pi\}, \quad \mathcal{E}_{\mathfrak{s}}^{t}:=\mathcal{E}^{t} \cap \mathcal{E}_{\mathfrak{s}} .
$$

8.8. The structure of $\mathcal{E}^{t}$. The following are some definitions and remarks regarding the structure of $\varepsilon^{t}$. The definitions here are made in a parallel fashion to those 
in 8] regarding Bernstein center, and most facts here can be deduced from [17] or 37.

(1) Let $\mathfrak{t}=(M, \tau)_{d}$ be a pair of a Levi subgroup and a discrete series on $M$. We will call such a pair a discrete pair. We say that two discrete pairs $(M, \tau)_{d}$ and $\left(M^{\prime}, \tau^{\prime}\right)_{d}$ are equivalent if there is a $g \in G$ and an unramified unitary character on $M$ such that $M={ }^{g} M^{\prime}$ and $\tau \otimes \chi \simeq{ }^{g} \tau^{\prime}$.

(2) Let $\mathfrak{B}_{d}$ be the set of equivalence classes of discrete pairs. Then, for any tempered representation $\pi$, there is a unique class $(M, \tau)_{d}$ in $\mathfrak{B}_{d}$ such that $\pi$ is a subquotient of a parabolically induced representation from $(M, \tau \otimes \chi)_{d}$ for some unramified unitary character of $M$. In this case, we will say that $(M, \tau)_{d}$ is the discrete support of $\pi$.

(3) For $\mathfrak{t} \in \mathfrak{B}_{d}$, let $\mathcal{E}^{t}(\mathfrak{t})$ be the set of irreducible tempered representations whose discrete supports are $\mathfrak{t}$. Then the result of [17] (see also [37, III.4.1]) says that $\mathcal{E}^{t}$ is the disjoint union of $\mathcal{E}^{t}(\mathfrak{t}), \mathfrak{t} \in \mathfrak{B}_{d}$, and the Plancherel formula can be written as follows: for $F \in C_{c}^{\infty}(G)$,

$$
F(1)=\int_{\mathcal{E}^{t}} \widehat{F}(\pi) d \pi=\sum_{\mathfrak{t} \in \mathfrak{B}_{d}} \int_{\mathcal{E}^{t}(\mathfrak{t})} \widehat{F}(\pi) d \pi .
$$

(4) Denote by $\operatorname{Irr}_{\mathfrak{t}}$ the set of all irreducibly induced representations $\operatorname{Ind}_{P}^{G} \tau$ from some $(M, \tau)_{d}$ in $\mathfrak{t}$. Then

$$
\int_{\mathcal{E}^{t}(\mathfrak{t})} \widehat{F}(\pi) d \pi=\int_{\operatorname{Irr}_{\mathfrak{t}}} \widehat{F}(\pi) d \pi
$$

and the Plancherel measure of $\mathcal{E}^{t}(\mathfrak{t}) \backslash \operatorname{Irr}_{\mathfrak{t}}$ is zero (see [37, IV.2.2, IV.3]).

(5) The representations in $\mathcal{E}^{t}(\mathfrak{t})$ have the same cuspidal support, that is, there is a pair $\left(M^{\prime}, \sigma\right)_{c}$ of a Levi subgroup $M^{\prime}$ and its supercuspidal representation $\sigma$ such that each representation in $\mathcal{E}^{t}(\mathfrak{t})$ is a subquotient of the parabolically induced representation from $\left(M^{\prime}, \sigma \otimes \chi\right)_{d}$ for some unramified character $\chi$ of $M^{\prime}$. Moreover, representations in $\mathcal{E}^{t}(\mathfrak{t})$ have the same depth ([31]).

We also need the following lemma for the proof of Lemma 8.10. Here, by parabolic induction, we mean unitary parabolic induction.

Lemma 8.9. Let $\mathfrak{t} \in \mathfrak{B}_{d}$. Let $\left(M_{1}, \tau_{1}\right)_{d}$ and $\left(M_{2}, \tau_{2}\right)_{d}$ be in the class of $\mathfrak{t}$. Then, any parabolically induced representations $\operatorname{Ind}_{P_{1}}^{G} \tau_{1}$ and $\operatorname{Ind}_{P_{2}}^{G} \tau_{2}$, where $P_{i}, i=1,2$, is a parabolic subgroup with Levi factor $M_{i}$, are isomorphic when restricted to any special maximal compact subgroup.

Proof. Let $K$ be a special maximal compact subgroup and $P_{0}$ a minimal parabolic subgroup of $G$ such that $G=P_{0} K=K P_{0}$. Without loss of generality, we may assume that $P_{1}$ and $P_{2}$ contain $P_{0}$.

Denote the set of unitary unramified characters of $M_{i}$ by $X^{u}\left(M_{i}\right), i=1,2$. Let $g \in G$ be such that ${ }^{g} M_{1}=M_{2}$ and ${ }^{g} \tau_{1} \simeq \tau_{2} \otimes \chi_{2}$ for some $\chi_{2} \in X^{u}\left(M_{2}\right)$.

Suppose $\tau_{1}$ is $G$-regular (that is, $\tau_{1} \not h_{\tau_{1}}$ for any $h$ normalizing $M_{1}$ ). Then, $\tau_{2} \otimes \chi_{2}$ is also regular and $\operatorname{Ind}_{P_{1}}^{G} \tau_{1} \simeq \operatorname{Ind}_{g_{1}}^{G} \tau_{2} \otimes \chi_{2} \simeq \operatorname{Ind}_{P_{2}}^{G} \tau_{2} \otimes \chi_{2}$. The second isomorphism follows from Frobenius reciprocity and [37, III.7.3]. Since

$$
\left(\operatorname{Ind}_{P_{2}}^{G} \tau_{2}\right)\left|K \simeq\left(\operatorname{Ind}_{P_{2}}^{G} \tau_{2} \otimes \chi_{2}\right)\right| K
$$

by [10, (3.1.1)], the lemma follows. 
In general, there is an unramified unitary character $\chi$ of $M_{1}$ such that $\tau_{1} \otimes \chi$ is $G$-regular This follows from the fact that $\left\{\chi \in X^{u}\left(M_{1}\right) \mid \tau_{1} \otimes \chi \simeq \tau_{1}\right\}$ is a finite set while $X^{u}\left(M_{1}\right)$ forms a complex manifold. Using [10, (3.1.1)], the general case follows from the $G$-regular case.

Lemma 8.10. Let $\Gamma \in \mathfrak{S}$ and $\mathfrak{t} \in \mathfrak{B}_{d}$. Then, $\mathcal{E}_{\Gamma}^{t}(\epsilon) \cap \mathcal{E}^{t}(\mathfrak{t})$ either contains $\operatorname{Irr}_{\mathfrak{t}}$ or it has Plancherel measure zero.

Proof. Suppose $\Gamma \sim 0$ and thus $\varepsilon_{\Gamma}^{t}(\epsilon)$ is the set of depth zero representations. By (5) of $8.8, \mathcal{E}_{\Gamma}^{t}(\epsilon) \cap \mathcal{E}^{t}(\mathfrak{t})$ is either $\mathcal{E}^{t}(\mathfrak{t})$ or empty.

Suppose $\Gamma \nsim 0$. Note that for any $\Sigma, L_{x, \epsilon}$ is contained in a special maximal compact subgroup. Then, by Lemma 8.9, if there is an irreducibly induced representation $\operatorname{Ind}_{P}^{G} \tau$ in $\mathcal{E}_{\Gamma}^{t}(\epsilon) \cap \mathcal{E}^{t}(\mathfrak{t})$ for some $(M, \tau)_{d}$ in $\mathfrak{t}$, $\operatorname{Irr}_{\mathfrak{t}}$ is contained in $\mathcal{E}_{\Gamma}^{t}(\epsilon) \cap \mathcal{E}^{t}(\mathfrak{t})$. Otherwise, $\mathcal{E}_{\Gamma}^{t}(\epsilon) \cap \mathcal{E}^{t}(\mathfrak{t})$ is contained in $\mathcal{E}^{t}(\mathfrak{t}) \backslash \operatorname{Irr}_{\mathfrak{t}}$ whose Plancherel measure is zero.

In the following proposition, we denote the union of $\mathcal{E}^{t}(\mathfrak{t}), \mathfrak{t} \in \mathfrak{B}_{d}$, with $\operatorname{Irr}_{\mathfrak{t}} \subset$ $\mathcal{E}_{\Gamma}^{t}(\epsilon) \cap \mathcal{E}^{t}(\mathfrak{t})$ by $\overline{\mathcal{E}_{\Gamma}^{t}}(\epsilon)$.

\section{Proposition 8.11.}

(1) For $\Gamma \in \mathfrak{S}$ and $F \in C_{c}^{\infty}(G)$, we have

$$
\int_{\overline{\varepsilon_{\Gamma}^{t}}(\epsilon)} \widehat{F}(\pi) d \pi=\int_{\varepsilon_{\Gamma}^{t}(\epsilon)} \widehat{F}(\pi) d \pi
$$

(2) For $F \in C_{c}^{\infty}(G)$, we have

$$
F(1)=\int_{\mathcal{E}^{t}} \widehat{F}(\pi) d \pi=\int_{\mathcal{E}^{t} \backslash\left(\stackrel{\cup}{\Gamma \in \mathcal{S}}_{\Gamma} \varepsilon_{\Gamma}^{t}(\epsilon)\right)} \widehat{F}(\pi) d \pi+\sum_{\Gamma \in \mathfrak{S}} \int_{\mathcal{E}_{\Gamma}^{t}(\epsilon)} \widehat{F}(\pi) d \pi .
$$

(3) Each $\mathcal{E}_{\Gamma}^{t}(\epsilon)$ has a finite Plancherel volume, that is, vol $\mathcal{E}^{u}\left(\mathcal{E}_{\Gamma}^{t}(\epsilon)\right)<\infty$.

Remark 8.12. Note that $\mathfrak{S}$ is a countable set. Moreover, for any $F \in C_{c}^{\infty}(G)$, $\int_{\mathcal{E}_{\Gamma}^{t}(\epsilon)} \widehat{F}(\pi) d \pi \neq 0$ for only finitely many $\Gamma \in \mathfrak{S}$. Hence, the $\sum$ in $(2)$ is well defined.

Proof. (1) This follows from Lemma 8.10 and (4) of 88.8

(2) We have

$$
\begin{aligned}
& \int_{\mathcal{E}^{t}} \widehat{F}(\pi) d \pi \stackrel{(i)}{=} \int_{\mathcal{E}^{t} \backslash\left(\stackrel{\circ}{\Gamma \in \mathcal{S}}_{\overline{\varepsilon_{\Gamma}^{t}}(\epsilon)}\right)} \widehat{F}(\pi) d \pi+\sum_{\Gamma \in \mathfrak{S}} \int_{\overline{\varepsilon_{\Gamma}^{t}}(\epsilon)} \widehat{F}(\pi) d \pi \\
& \stackrel{(i i)}{=} \int_{\mathcal{E}^{t} \backslash\left(\stackrel{\bigcirc}{\Gamma \in \mathcal{S}}_{\Gamma} \varepsilon_{\Gamma}^{t}(\epsilon)\right)} \widehat{F}(\pi) d \pi+\sum_{\Gamma \in \mathfrak{S}} \int_{\mathcal{E}_{\Gamma}^{t}(\epsilon)} \widehat{F}(\pi) d \pi .
\end{aligned}
$$

The equality ( $i$ ) follows from (1) and the decomposition in (3) in 8.8 , Since $\left(\overline{\varepsilon_{\Gamma}^{t}}(\epsilon) \cup\right.$ $\left.\varepsilon_{\Gamma}^{t}(\epsilon)\right) \backslash\left(\overline{\varepsilon_{\Gamma}^{t}}(\epsilon) \cap \mathcal{E}_{\Gamma}^{t}(\epsilon)\right)$ has Plancherel measure zero by Lemma 8.10 combining this with (1), the equality (ii) follows.

(3) Let $x \in \mathcal{B}(G)$. Since any $\left(\pi, V_{\pi}\right) \in \mathcal{E}_{\Gamma}^{t}(\epsilon)$ is of depth $r_{d}, \mathcal{E}_{\Gamma}^{t}(\epsilon) \subset \mathcal{E}_{\left(G_{x, r_{d}+r^{\prime}}, 1\right)}$ for a sufficiently large $r^{\prime} \in \mathbb{R}$. Then, (3) is a result of the following lemma.

Lemma 8.13. For any open compact subgroup $J \subset G$, let $\mathcal{E}_{(J, 1)}^{t}=\left\{\left(\pi, V_{\pi}\right) \in \mathcal{E}^{t} \mid\right.$ $\left.V_{\pi}^{(J, 1)} \neq 0\right\}$. Then, $\mathcal{E}_{(J, 1)}^{t}$ has a finite Plancherel volume. 
Proof. Let $F \in C_{c}^{\infty}(G)$ be given by the characteristic function of $J$ divided by $\operatorname{vol}_{G}(J)$. Then, from the Plancherel formula on $G$, we have

$$
\begin{aligned}
\operatorname{vol}_{\mathcal{E}^{u}}\left(\mathcal{E}_{(J, 1)}^{t}\right) & \leq \int_{\mathcal{E}_{(J, 1)}^{t}} \operatorname{dim}_{\mathbb{C}}\left(V_{\pi}^{J}\right) d \pi \\
& =\int_{\mathcal{E}^{t}} \operatorname{dim}_{\mathbb{C}}\left(V_{\pi}^{J}\right) d \pi=\int_{\mathcal{E}^{t}} \widehat{F}(\pi) d \pi=F(1)=\frac{1}{\operatorname{vol}_{G}(J)} .
\end{aligned}
$$

\section{REVIEW ON T-ASYMPTOTIC EXPANSIONS}

As a preparation for the proof of Theorem 10.1, we review $\Gamma$-asymptotic expansions and related materials from [27.

Let $\epsilon \in \mathbb{R}$ be as before. Fix a strongly good $G$-datum of positive depth $\Sigma=$ $(\overrightarrow{\mathbf{G}}, y, \vec{r}, \vec{\phi})$, and let $\Gamma=\Gamma_{\Sigma}$. In this case, set $r_{-1}:=-\epsilon$. Associated to $\Gamma$, we have a subspace $\mathfrak{g}^{\Gamma}$ of invariant distributions on $\mathfrak{g}$.

9.1. Recall that $\Gamma^{-1}=\Gamma^{0}$. Let $\mathcal{N}^{i}:=\mathcal{N} \cap \mathfrak{g}^{i}, 0 \leq i \leq d$, and let $\mathcal{N}^{-1}=\mathcal{N}^{0}$. Then $\mathcal{N}^{i}$ is the set of nilpotent elements in $\mathfrak{g}^{i}$. Let $\Upsilon_{\Sigma}$ be the set of all triples $(i, x, s)$ with $i \in\{0, \ldots, d\}, x \in \mathcal{B}\left(\mathbf{G}^{i}, k\right)$, and $r_{i-1} \leq s<r_{i}$ if $i<d, r_{d-1} \leq s<\infty$ if $i=d$ :

$$
\begin{aligned}
\Upsilon_{\Sigma}:=\left\{(i, x, s) \in \mathbb{Z} \times \mathcal{B}(\mathbf{G}, k) \times \tilde{\mathbb{R}} \mid i \in\{0, \ldots, d\}, x \in \mathcal{B}\left(\mathbf{G}^{i}, k\right),\right. \\
\left.r_{i-1} \leq s<r_{i} \text { if } i \neq d, r_{d-1} \leq s<\infty \text { if } i=d\right\} .
\end{aligned}
$$

Let $\mathcal{J}(\mathfrak{g})$ denote the set of $G$-invariant distributions on $\mathfrak{g}$. In the following, we define a subspace $\mathcal{J}^{\Gamma}$ of $\mathcal{J}(\mathfrak{g})$.

\section{Definition 9.2.}

(1) Let $(i, x, s) \in \Upsilon_{\Sigma}$ with $s \leq r_{d-1}$. Recall that $\mathcal{L}_{x}^{i}$ is defined in $\S 4.3(1)$, and define $d_{x,-s}^{i, \Gamma}$ as follows: If $r_{i-1}<s<r_{i}$, let

$$
\begin{aligned}
\partial_{x,-s}^{i, \Gamma}:=\{\mathrm{T} \in \mathcal{J}(\mathfrak{g}) \mid & \text { if } f \in C\left(\left(\Gamma^{i}+\mathfrak{g}_{x,-s}^{i}+\mathcal{L}_{x}^{i \sharp}\right) / \mathcal{L}_{x}^{i \sharp}\right) \text { and } \\
& \left.\operatorname{Supp}(f) \cap\left(\Gamma^{i}+\mathfrak{g}_{(-s)^{+}}^{i}\right)=\emptyset, \text { then } \mathrm{T}(f)=0\right\} .
\end{aligned}
$$

Let

$$
\begin{aligned}
\mathcal{J}_{x,-r_{i-1}}^{i, \Gamma}:=\left\{\mathrm{T} \in \mathcal{J}(\mathfrak{g}) \mid \text { if } f \in C\left(\left(\Gamma^{i}+\mathfrak{g}_{x,-r_{i-1}}^{i}+\mathcal{L}_{x}^{i \sharp}\right) / \mathcal{L}_{x}^{i \sharp}\right)\right. \text { and } \\
\left.\quad \operatorname{Supp}(f) \cap G^{i}\left(\Gamma^{i-1}+\mathfrak{g}_{\left(-r_{i-1}\right)^{+}}^{i-1}\right)=\emptyset, \text { then } \mathrm{T}(f)=0\right\} .
\end{aligned}
$$

(2) If $(i, x, s) \in \Upsilon_{\Sigma}$ and $s>r_{d-1}$, then $i=d$. In this case, let

$$
\begin{aligned}
\partial_{x,-s}^{d, \Gamma}:=\{\mathrm{T} \in \mathcal{J}(\mathfrak{g}) \mid & \text { if } f \in C\left(\left(\Gamma^{d}+\mathfrak{g}_{x,-s}\right) / \mathfrak{g}_{x,-s_{d-1}}\right) \text { and } \\
& \left.\operatorname{Supp}(f) \cap\left(\Gamma^{d}+\mathfrak{g}_{(-s)^{+}}\right)=\emptyset, \text { then } \mathrm{T}(f)=0\right\} .
\end{aligned}
$$

(3) Set

$$
\partial^{\Gamma}:=\bigcap_{(i, x, s) \in \Upsilon_{\Sigma}} J_{x,-s}^{i, \Gamma} .
$$

For any $t \in \tilde{\mathbb{R}}$, define a subspace $D_{t}$ of $C_{c}^{\infty}(\mathfrak{g})$ as follows:

$$
D_{t}:=\sum_{x \in \mathcal{B}(\mathbf{G}, k)} C_{c}\left(\mathfrak{g} / \mathfrak{g}_{x, t}\right) .
$$


Note that $D_{t}$ is the space consisting of Fourier transforms of $f \in C_{c}^{\infty}\left(\mathfrak{g}_{t^{*}}\right)$ (2, $(4.2 .3)])$.

Theorem 9.3 ([27, (8.1.1)]). Suppose $(\mathrm{H} k)$ and $(\mathrm{H \mathcal {N }})$ are valid. Let $\Gamma$ be as above. Let $\mathcal{J}_{\mathcal{O}(\Gamma)}$ be the linear span of orbital integrals $\mu_{\mathcal{O}}$ associated to $\mathcal{O}$ with $\mathcal{O} \in \mathcal{O}(\Gamma)$. Then, we have $\mathcal{J}^{\Gamma} \equiv \mathcal{J}_{\mathcal{O}(\Gamma)}$ on $D_{-s_{d-1}}$.

For $\left(\pi, V_{\pi}\right) \in \mathcal{E}$, we define the Fourier transform $\widehat{\Theta}_{\pi} \in \mathcal{J}(\mathfrak{g})$ of $\Theta_{\pi}$ as follows: for $f \in C_{c}^{\infty}(\mathfrak{g})$, let $f_{0^{+}}=f \cdot\left[\mathfrak{g}_{0^{+}}\right]$and

$$
\widehat{\Theta}_{\pi}(f)=\Theta_{\pi}\left(\widehat{f_{0^{+}}} \circ \log \right) .
$$

Theorem $9.4([27,(8.2 .3)])$. Let $\left(\pi, V_{\pi}\right) \in \mathcal{E}$. Let $\Sigma=(\overrightarrow{\mathbf{G}}, y, \vec{r}, \vec{\phi})$ be a strongly good $G$-datum of positive depth. Assume the hypotheses $(\mathrm{HB}),(\mathrm{H} k)$ and $(\mathrm{HN})$ are valid. Let $x \in \mathcal{B}\left(\mathbf{G}^{0}, k\right)$ and $\Gamma:=\Gamma_{\Sigma}$. Let $\mathcal{L}_{x}$ and $L_{x}$ be as in $\$ 5.12$. Let $X \in \mathfrak{g}_{x,-\epsilon}^{0} \cap \mathfrak{g}_{(-\epsilon)}^{0}$, and let $\chi_{\Gamma+X}$ be the character of $L_{x}$ with the dual blob $\Gamma+X+\mathcal{L}_{x}^{\sharp}$. Suppose $\left(\pi, V_{\pi}\right)$ contains $\left(L_{x}, \chi_{\Gamma+X}\right)$. Then,

(1) $\widehat{\Theta}_{\pi} \in \mathcal{J}^{-\Gamma}$,

(2) $\widehat{\Theta}_{\pi}$ is $\Gamma$-asymptotic on $\mathfrak{g}_{s_{d-1}^{+}}$; that is, there are $c_{\mathcal{O}}(\pi) \in \mathbb{C}$ indexed by $\mathcal{O}(\Gamma)$ such that $\Theta_{\pi}(f \circ \log )=\sum_{\mathcal{O} \in \mathcal{O}(\Gamma)} c_{\mathcal{O}}(\pi) \cdot \widehat{\mu_{\mathcal{O}}}(f)$ for all $f \in C_{c}^{\infty}\left(\mathfrak{g}_{s_{d-1}^{+}}\right)$, where $\mu_{\mathcal{O}}$ is the orbital integral associated to $\mathcal{O} \in \mathcal{O}(\Gamma)$.

9.5. Recall that $\mathbf{G}^{0}=\mathbf{C}_{\mathbf{G}}(\Gamma)$. Label the elements of $\mathcal{O}\left(G^{0}, 0\right)$, the set of nilpotent orbits in $\mathfrak{g}^{0}$, as $\mathcal{O}_{1}^{0}, \ldots, \mathcal{O}_{m}^{0}$. Because $\Gamma$ is semisimple, the Jordan decomposition gives a bijection between $\mathcal{O}(\Gamma)$ and $\mathcal{O}\left(G^{0}, 0\right)$ (see 2.6 ). If $\mathcal{O}^{0} \in \mathcal{O}\left(G^{0}, 0\right)$, the corresponding element of $\mathcal{O}(\Gamma)$ is ${ }^{G}\left(\Gamma+\mathcal{O}^{0}\right)$. Let $\mathcal{O}_{i}={ }^{G}\left(\Gamma+\mathcal{O}_{i}^{0}\right), 1 \leq i \leq m$.

In [27, we also found some test functions $f_{i}^{\Gamma}, \mathcal{O}_{i} \in \mathcal{O}(\Gamma)$, such that $(i)$ the obvious pairing on $\left\langle\mu_{\mathcal{O}_{i}} \mid \mathcal{O}_{i} \in \mathcal{O}(\Gamma)\right\rangle \times\left\langle f_{i}^{\Gamma} \mid \mathcal{O}_{i} \in \mathcal{O}(\Gamma)\right\rangle$ is nondegenerate, and (ii) for each $\mathcal{O}_{i} \in \mathcal{O}(\Gamma)$, there are $x_{i} \in \mathcal{B}\left(\mathbf{G}^{0}, x\right)$ and $X_{i} \in \mathcal{O}_{i} \cap \mathfrak{g}_{x_{i},(-\epsilon)}^{0}$ with $f_{i}^{\Gamma}=\left[\Gamma+X_{i}+\mathcal{L}_{x_{i}}^{\sharp}\right]$.

This provides a way to compute the coefficients $c_{\mathcal{O}}(\pi)$ in Theorem 9.4 by linear algebra. Moreover, if $\mathrm{T} \in \mathcal{J} \Gamma$, then the restriction of $\mathrm{T}$ to $D_{-s_{d-1}}$ is completely determined by $\mathrm{T}\left(f_{i}^{\Gamma}\right), i=1, \cdots, m$, in the following sense.

Theorem 9.6 ([27, (9.1.6)]). Suppose $(\mathrm{H} k)$ and $(\mathrm{HN})$ are valid. Let $\mathrm{T} \in \mathrm{J}^{\Gamma}$. Let $f_{i}^{\Gamma}$ be as in 99.5 . If $\mathrm{T}\left(f_{i}^{\Gamma}\right)=0$ for all $i=1, \cdots, m$, then $\mathrm{T} \equiv 0$ on $D_{-s_{d-1}}$.

Corollary 9.7. Suppose $(\mathrm{H} k)$ and $(\mathrm{HN})$ are valid. Let $\mathrm{T}_{1}, \mathrm{~T}_{2} \in \mathfrak{j}^{\Gamma}$. If

$$
\mathrm{T}_{1}\left(\left[\Gamma+X+\mathcal{L}_{x}^{\sharp}\right]\right)=\mathrm{T}_{2}\left(\left[\Gamma+X+\mathcal{L}_{x}^{\sharp}\right]\right)
$$

for each $x \in \mathcal{B}\left(\mathbf{G}^{0}, k\right)$ and $X \in \mathcal{N}^{0} \cap \mathfrak{g}_{x,(-\epsilon)}^{0}$, then $\mathrm{T}_{1} \equiv \mathrm{T}_{2}$ on $D_{-s_{d-1}}$.

Proof. Since

$$
\left\{f_{i}^{\Gamma}\right\}_{i=1}^{m} \subset\left\{\left[\Gamma+X+\mathcal{L}_{x}^{\sharp}\right] \mid x \in \mathcal{B}\left(\mathbf{G}^{0}, k\right), X \in \mathcal{N}^{0} \cap \mathfrak{g}_{x,(-\epsilon)}^{0}\right\},
$$

the corollary follows from the above theorem.

\section{MATChing}

In the following theorem, we match the spectral decomposition factors in Corollary 7.7 and Proposition 8.11. This is a crucial step in proving Theorem 11.1. Recall that if $\Gamma \sim 0, s_{d-1}=s_{-1}=0$. 
Theorem 10.1. Suppose $(\mathrm{H} k),(\mathrm{HB}),(\mathrm{HGT})$ and $(\mathrm{H} \mathcal{N})$ are valid. Let $\Gamma \in \mathfrak{S}$. Then for any $f \in C_{c}^{\infty}\left(\mathfrak{g}_{s_{d-1}^{+}}\right)$, we have

$$
\int_{\mathfrak{g}_{\Gamma}} \widehat{f}(X) d X=\int_{\mathcal{E}_{\Gamma}^{t}(\epsilon)} \widehat{f \circ \log }(\pi) d \pi .
$$

Proof. Suppose $\Gamma \sim 0$. Then $\mathfrak{g}_{\Gamma}=\mathfrak{g}_{0}$ and $\mathcal{E}_{\Gamma}^{t}(\epsilon)$ is the set of all tempered depth zero representations. Hence the above equality follows from [25, (3.3.1)].

Now assume that $\mathrm{d}(\Gamma)<0$. Without loss of generality, we may assume $\Gamma=\Gamma^{0}$. Write $\Gamma$ as in 7.1

$$
\Gamma=\Gamma^{0}=\Gamma_{d}+\Gamma_{d-1}+\cdots+\Gamma_{0}
$$

Let $\mathrm{T}_{\Gamma}^{\mathfrak{g}}$ and $\mathrm{T}_{\Gamma}^{G}$ be invariant distributions defined as follows: for $f \in C_{c}^{\infty}(\mathfrak{g})$, let $f_{0^{+}}:=f \cdot\left[\mathfrak{g}_{0^{+}}\right]$. Then,

$$
\begin{aligned}
& \mathrm{T}_{\Gamma}^{\mathfrak{g}}(f):=\int_{\mathfrak{g}_{\Gamma}} \widehat{f_{0^{+}}}(X) d X, \\
& \mathrm{~T}_{\Gamma}^{G}(f):=\int_{\mathcal{E}_{\Gamma}^{t}(\epsilon)} \widehat{f_{0^{+}} \circ \log }(\pi) d \pi=\int_{\mathcal{E}_{\Gamma}^{t}(\epsilon)} \Theta_{\pi}\left(f_{0^{+}} \circ \log \right) d \pi .
\end{aligned}
$$

Hence, to prove the theorem, it is enough to prove that

$$
\mathrm{T}_{\Gamma}^{\mathfrak{g}}(\widehat{f})=\mathrm{T}_{\Gamma}^{G}(\widehat{f})
$$

for any $f \in D_{-s_{d-1}}$, since $\widehat{D}_{-s_{d-1}}=C_{c}^{\infty}\left(\mathfrak{g}_{s_{d-1}^{+}}\right)$. We first need the following lemma.

Lemma 10.2. $\widehat{\mathrm{T}}_{\Gamma}^{\mathfrak{g}}$ and $\widehat{\mathrm{T}}_{\Gamma}^{G}$ are elements of $\mathcal{J}^{-\Gamma}$.

Proof. By Theorem 9.4(1), we have $\widehat{T}_{\Gamma}^{G} \in \mathcal{J}^{-\Gamma}$. To show $\widehat{T}_{\Gamma}^{\mathfrak{g}} \in \mathcal{J}^{-\Gamma}$, note that for any $f \in C_{c}^{\infty}(\mathfrak{g})$, we have

$$
\widehat{\mathrm{T}}_{\Gamma}^{\mathfrak{g}}(f)=\int_{\mathfrak{g}_{\Gamma}} f(-X) d X=\int_{-\mathfrak{g}_{\Gamma}} f(X) d X
$$

and

$$
\operatorname{Supp}\left(\widehat{T}_{\Gamma}^{\mathfrak{g}}\right)=-\mathfrak{g}_{\Gamma}=\mathfrak{g}_{-\Gamma} .
$$

If $s>r_{d-1}$, then

$$
\begin{aligned}
\operatorname{Supp}\left(\widehat{\mathrm{T}}_{\Gamma}^{\mathfrak{g}}\right) & =-\mathfrak{g}_{\Gamma}={ }^{G}\left(-\Gamma+\mathfrak{g}_{0}^{0}\right) \subset{ }^{G}\left(-\Gamma+\mathfrak{g}_{(-s)^{+}}^{d}\right)={ }^{G}\left(-\Gamma_{d}+\mathfrak{g}_{(-s)^{+}}^{d}\right) \\
& =-\Gamma_{d}+\mathfrak{g}_{(-s)^{+}}^{d} .
\end{aligned}
$$

Hence $\widehat{\mathrm{T}}_{\Gamma}^{\mathfrak{g}} \in \mathrm{J}_{x,-s}^{d,-\Gamma}$. If $r_{i-1}<s \leq r_{i}, i=0, \cdots, d$, then

$$
\operatorname{Supp}\left(\widehat{T}_{\Gamma}^{\mathfrak{g}}\right)={ }^{G}\left(-\Gamma+\mathfrak{g}_{0}^{0}\right) \subset{ }^{G}\left(-\Gamma+\mathfrak{g}_{(-s)^{+}}^{i}\right)={ }^{G}\left(-\Gamma^{i}+\mathfrak{g}_{(-s)^{+}}^{i}\right),
$$

which implies that $\widehat{T}_{\Gamma}^{\mathfrak{g}} \in \mathrm{J}_{x,-s}^{i,-\Gamma}$. Therefore, $\widehat{\mathrm{T}}_{\Gamma}^{\mathfrak{g}} \in \bigcap_{(i, x, s) \in \Upsilon_{\Gamma}} \partial_{x,-s}^{i,-\Gamma}=\mathcal{J}^{-\Gamma}$.

Continuing with the proof of Theorem 10.1 by Lemma 10.2 and Corollary 9.7 . it is enough to check that for any $x \in \mathcal{B}\left(\mathbf{G}^{0}, k\right)$ and $X \in \mathcal{N}^{0} \cap \mathfrak{g}_{x,-\epsilon}^{0}$,

(E) $\quad \widehat{\mathrm{T}}_{\Gamma}^{\mathfrak{g}}\left(\left[-\Gamma+X+\mathcal{L}_{x}^{\sharp}\right]\right)=\widehat{\mathrm{T}}_{\Gamma}^{G}\left(\left[-\Gamma+X+\mathcal{L}_{x}^{\sharp}\right]\right)$.

We first note that $\operatorname{Supp}\left(\left[-\Gamma+X+\mathcal{L}_{x}^{\sharp}\right]\right) \subset{ }^{G}\left(-\Gamma+X+\mathfrak{g}_{0}^{0}\right) \subset{ }^{G}\left(-\Gamma+\mathfrak{g}_{0}^{0}\right)=\mathfrak{g}_{-\Gamma}$. The first inclusion follows from Lemma 5.13, and the second inclusion follows from $X$ being nilpotent and thus $X \in \mathfrak{g}_{0}^{0}$. Then we have

$$
\widehat{T}_{\Gamma}^{\mathfrak{g}}\left(\left[-\Gamma+X+\mathcal{L}_{x}^{\sharp}\right]\right)=\operatorname{vol}_{\mathfrak{g}}\left(\mathcal{L}_{x}^{\sharp}\right)=\frac{1}{v o l_{\mathfrak{g}}\left(\mathcal{L}_{x}\right)} .
$$


Computing $\widehat{\mathrm{T}}_{\Gamma}^{G}\left(\left[-\Gamma+X+\mathcal{L}_{x}^{\sharp}\right]\right)$, write $f$ for $\left[-\Gamma+X+\mathcal{L}_{x}^{\sharp}\right]$. Then,

$$
\widehat{f}(Y)=\frac{1}{\operatorname{vol}_{G}\left(L_{x}\right)} \cdot \Lambda(\mathrm{B}(-\Gamma+X, Y)) \cdot\left[\mathcal{L}_{x}\right](Y) \text {. }
$$

Note that $\left(\operatorname{vol}_{G}\left(L_{x}\right) \cdot \widehat{f} \circ \log \right)$ is a character of $L_{x}$ with dual blob $-\Gamma+X+\mathcal{L}_{x}^{\sharp}$, that is, $\left(\operatorname{vol}_{G}\left(L_{x}\right) \cdot \widehat{f} \circ \log \right)=\chi_{-\Gamma+X}$ on $L_{x}$. Then $\Theta_{\pi}(\widehat{f} \circ \log )=\Theta_{\pi}\left(\chi_{-\Gamma+X}\right)$ is the multiplicity $m\left(\chi_{\Gamma-X}, V_{\pi}\right)$ of $\chi_{\Gamma-X}$ in $V_{\pi}$. Since $m\left(\chi_{\Gamma-X}, V_{\pi}\right)=0$ unless $\pi \in \mathcal{E}_{\Gamma}^{t}(\epsilon)$, we have

$$
\begin{aligned}
\frac{1}{\operatorname{vol}_{\mathfrak{g}}\left(\mathcal{L}_{x}\right)} & =\widehat{f}(\log (1))=\int_{\mathcal{E}^{t}} \Theta_{\pi}(\widehat{f} \circ \log ) d \pi \\
& =\int_{\mathcal{E}^{t}} m\left(\chi_{\Gamma-X}, V_{\pi}\right) d \pi=\int_{\mathcal{E}_{\Gamma}^{t}(\epsilon)} m\left(\chi_{\Gamma-X}, V_{\pi}\right) d \pi \\
& =\int_{\mathcal{E}_{\Gamma}^{t}(\epsilon)} \Theta_{\pi}(\widehat{f} \circ \log ) d \pi=\widehat{\mathrm{T}}_{\Gamma}^{G}\left(\left[-\Gamma+X+\mathcal{L}_{x}^{\sharp}\right]\right) .
\end{aligned}
$$

Now the equality (E) follows from (a) and (b), and Theorem 10.1 is proved.

\section{TEMPERED REPRESENTATIONS}

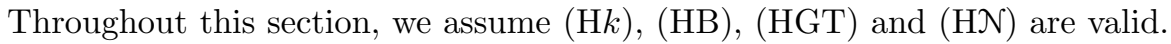

We prove that for almost every tempered representation $\left(\pi, V_{\pi}\right)$, there is a strongly good $G$-datum $\Sigma$ such that $\left(\pi, V_{\pi}\right)$ contains the K-type $\left(K_{\Sigma}^{+}, \phi_{\Sigma}\right)$. As a corollary of the proof, we get a spectral decomposition of the delta distribution on $G$, where each decomposition factor is parameterized by an element of $\mathfrak{S}$.

Theorem 11.1. Almost every tempered representation is an element of $\mathcal{E}_{\Gamma}^{t}(\epsilon)$ for some $\Gamma \in \mathfrak{S}$. That is, $\mathcal{E}^{t} \backslash \bigcup_{\Gamma \in \mathfrak{S}} \mathcal{E}_{\Gamma}^{t}(\epsilon)$ has Plancherel measure zero.

In the following, for $\mathcal{A}, \mathcal{A}^{\prime} \subset \mathcal{E}^{t}$, if $\left(\mathcal{A} \cup \mathcal{A}^{\prime}\right) \backslash\left(\mathcal{A}^{\prime} \cap \mathcal{A}\right)$ has measure zero, we will write $\mathcal{A} \stackrel{\underline{\mu}}{=} \mathcal{A}^{\prime}$.

Proof. We fix $x \in \mathcal{B}(\mathbf{G}, k)$. For $\alpha \in \mathbb{Z}_{>0}$, let $F_{\alpha}:=\left[G_{x, \alpha^{+}}\right]$. Then, for any $\left(\pi, V_{\pi}\right) \in \mathcal{E}$, we have $\widehat{F_{\alpha}}(\pi)=\widehat{\Theta}_{\pi}\left(\left[G_{x, \alpha^{+}}\right]\right)=\operatorname{vol}_{G}\left(G_{x, \alpha^{+}}\right) \operatorname{dim} V_{\pi}^{\left(G_{x, \alpha^{+}}, 1\right)} \geq 0$. Let $\mathcal{E}^{t \alpha}:=\mathcal{E}_{\left(G_{x, \alpha}, 1\right)}^{t}$, and let $\mathcal{E}_{\Gamma}^{t}(\epsilon)^{\alpha}:=\mathcal{E}_{\Gamma}(\epsilon) \cap \mathcal{E}^{t \alpha}$. Then we have

$$
\begin{aligned}
& \int_{\mathfrak{g}} \widehat{F_{\alpha} \circ \exp }(X) d X=\int_{\mathcal{E}^{t}} \widehat{F_{\alpha}}(\pi) d \pi=\int_{\mathcal{E}^{t \alpha}} \widehat{F_{\alpha}}(\pi) d \pi \stackrel{(i)}{\geq} \sum_{\Gamma \in \mathfrak{S}} \int_{\mathcal{E}_{\Gamma}^{t}(\epsilon)^{\alpha}} \widehat{F_{\alpha}}(\pi) d \pi \\
& =\sum_{\Gamma \in \mathfrak{S}, \mathcal{E}_{\Gamma}^{t}(\epsilon)^{\alpha} \neq \emptyset} \int_{\mathcal{E}_{\Gamma}^{t}(\epsilon)^{\alpha}} \widehat{F_{\alpha}}(\pi) d \pi \stackrel{(i i)}{=} \sum_{\Gamma \in \mathfrak{S}, \varepsilon_{\Gamma}^{t}(\epsilon)^{\alpha} \neq \emptyset} \int_{\mathfrak{g}_{\Gamma}} \widehat{F_{\alpha} \circ \exp }(X) d X \\
& \stackrel{(i i i)}{=} \sum_{\Gamma \in \mathfrak{S}} \int_{\mathfrak{g}_{\Gamma}} \widehat{F_{\alpha} \circ \exp }(X) d X \stackrel{(i v)}{=} \int_{\mathfrak{g}} \widehat{F_{\alpha} \circ \exp }(X) d X .
\end{aligned}
$$

The relations (i), (iii) and (iv) are straightforward. We use Theorem 10.1 to verify (ii).

The first inequality $(i)$ follows from $\bigcup_{\Gamma \in \mathfrak{S}} \varepsilon_{\Gamma}^{t}(\epsilon)^{\alpha} \subset \mathcal{E}^{t \alpha}$ and $\widehat{F_{\alpha}}(\pi) \geq 0$.

To prove $(i i)$, it is enough to verify that for $\Gamma \in \mathfrak{S}$, if $\mathcal{E}_{\Gamma}^{t}(\epsilon)^{\alpha} \neq \emptyset$,

$$
\int_{\mathcal{E}_{\Gamma}^{t}(\epsilon)^{\alpha}} \widehat{F_{\alpha}}(\pi) d \pi=\int_{\mathcal{E}_{\Gamma}^{t}(\epsilon)} \widehat{F_{\alpha}}(\pi) d \pi=\int_{\mathfrak{g}_{\Gamma}} \widehat{F_{\alpha} \circ \exp }(X) d X .
$$

Since for $\left(\pi, V_{\pi}\right) \in \mathcal{E}_{\Gamma}^{t}(\epsilon), \widehat{F_{\alpha}}(\pi) \neq 0$ only if $\left(\pi, V_{\pi}\right) \in \mathcal{E}_{\Gamma}^{t}(\epsilon)^{\alpha}$, the first equality follows. To prove the second one, we note that $\widehat{F_{\alpha}}(\pi) \neq 0$ only if $\alpha \geq \varrho(\pi)=r_{d}$ 
for $\pi \in \mathcal{E}_{\Gamma}^{t}(\epsilon)$. Hence $\mathcal{E}_{\Gamma}^{t}(\epsilon)^{\alpha} \neq \emptyset$ only if $\alpha \geq r_{d}$. In that case, since $F_{\alpha} \circ \exp \epsilon$ $C_{c}^{\infty}\left(\mathfrak{g}_{r_{d}^{+}}\right) \subset C_{c}^{\infty}\left(\mathfrak{g}_{s_{d-1}^{+}}\right)$, the equality in Theorem 10.1 is valid for $F_{\alpha}$. Hence the above equalities are valid, and so is $(i i)$.

To prove (iii), we note that $\widehat{F_{\alpha} \circ \exp }$ is a scalar multiple of the characteristic function of $\mathfrak{g}_{x,-\alpha}$. Then since $\operatorname{Supp}\left(\widehat{F_{\alpha} \circ \exp }\right) \cap \mathfrak{g}_{\Gamma}=\mathfrak{g}_{x,-\alpha} \cap \mathfrak{g}_{\Gamma} \neq \emptyset$ only if $\mathcal{E}_{\Gamma}^{t}(\epsilon)^{\alpha} \neq$ $\emptyset$, the equality (iii) holds. The equality $(i v)$ follows from Lemma 7.5

Hence, $\geq$ in $(i)$ is in fact an equality for all $\alpha \in \mathbb{Z}_{>0}$. Therefore, $\varepsilon^{t \alpha} \underline{\underline{\mu}}$ $\bigcup_{\Gamma \in \mathfrak{S}} \mathcal{E}_{\Gamma}^{t}(\epsilon)^{\alpha}$. Since $\mathcal{E}^{t}=\bigcup_{\alpha \in \mathbb{Z}_{>0}} \mathcal{E}^{t \alpha}$ and $\mathcal{E}_{\Gamma}^{t}(\epsilon)=\bigcup_{\alpha \in \mathbb{Z}_{>0}} \mathcal{E}_{\Gamma}^{t}(\epsilon)^{\alpha}$, we can now conclude

(*)

$$
\mathcal{E}^{t} \stackrel{\mu}{=} \bigcup_{\Gamma \in \mathfrak{S}} \mathcal{E}_{\Gamma}^{t}(\epsilon) .
$$

Combining this with Lemma 8.6. we get that the above is a disjoint union.

Corollary 11.2. For any $f \in C_{c}^{\infty}(G)$, we have

$$
f(1)=\sum_{\Gamma \in \mathfrak{S}} \int_{\mathcal{E}_{\Gamma}^{t}} \Theta_{\pi}(f) d \pi
$$

Proof. This follows from Proposition $\underline{8.11}(1)$ and $\mathcal{E}^{t} \stackrel{\underline{\mu}}{=} \stackrel{0}{\Gamma \in \mathfrak{S}}_{\Gamma} \mathcal{E}_{\Gamma}^{t}$.

Remark 11.3. Note that $\mathcal{E}^{t} \stackrel{\mu}{=} \bigcup_{\Gamma \in \mathfrak{S}} \mathcal{E}_{\Gamma}^{t}(\epsilon)$ independent of the choice of $\epsilon>0$ such that $\mathfrak{g}_{\epsilon}=\mathfrak{g}_{0^{+}}$. Since $\mathcal{E}_{\Gamma}^{t}\left(\epsilon^{\prime}\right) \subset \mathcal{E}_{\Gamma}^{t}(\epsilon)$ for $0<\epsilon^{\prime}<\epsilon$ and the above $(*)$ is a disjoint union, $\mathcal{E}_{\Gamma}^{t}(\epsilon) \stackrel{\mu}{=} \mathcal{E}_{\Gamma}^{t}\left(\epsilon^{\prime}\right)$. By Lemma 8.10, we also have

$$
\bigcup_{\epsilon} \mathcal{E}_{\Gamma}^{t}(\epsilon) \stackrel{\mu}{=} \bigcap_{\epsilon} \mathcal{E}_{\Gamma}^{t}(\epsilon)
$$

where $\epsilon$ runs over real numbers such that $\mathfrak{g}_{\epsilon}=\mathfrak{g}_{0^{+}}$, and thus

$$
\mathcal{E}^{t} \stackrel{\mu}{=} \bigcup_{\Gamma \in \mathfrak{S}}^{\circ}\left(\bigcap_{\epsilon} \mathcal{E}_{\Gamma}^{t}(\epsilon)\right) \text {. }
$$

Theorem 11.4. For $\left(\pi, V_{\pi}\right) \in \bigcup_{\Gamma \in \mathfrak{S}}\left(\bigcap_{\epsilon} \mathcal{E}_{\Gamma}^{t}(\epsilon)\right)$, there is a strongly good $G$-datum $\Sigma$ such that $\left(\pi, V_{\pi}\right)$ contains $\left(K_{\Sigma}^{+}, \phi_{\Sigma}\right)$.

Proof. This is obvious if $\varrho(\pi)=0$. Now, suppose $\varrho(\pi)>0$. Let $\Gamma \not 0$ be such that $\left(\pi, V_{\pi}\right) \in \bigcap_{\epsilon} \mathcal{E}_{\Gamma}^{t}(\epsilon)$ and it splits over a tamely ramified extension $E$. Let $\overrightarrow{\mathbf{G}}, \vec{r}$ and $\vec{\phi}$ be the sequences associated to $\Gamma$. Fix a subset $D$ in $\mathcal{B}\left(\mathbf{G}^{0}, k\right)$ such that $D$ is compact and $G^{0} \cdot D=\mathcal{B}\left(\mathbf{G}^{0}, k\right)$. If $\mathbf{G}^{0}$ is semisimple, the closure of a chamber in $\mathcal{B}\left(\mathbf{G}^{0}, k\right)$ is such a subset. Otherwise, since $\mathcal{B}\left(\mathbf{G}^{0}, k\right)$ is the product of $\mathcal{B}\left(\mathbf{G}^{\text {0der }}, k\right)$ and $\mathbf{X}_{*}\left(\mathbf{Z}_{\mathbf{G}^{0}}, k\right) \otimes \mathbb{R}$, and since $\left(\mathbf{X}_{*}\left(\mathbf{Z}_{\mathbf{G}^{0}}, k\right) \otimes \mathbb{R}\right) / Z_{G^{0}}$ is compact, such a subset $D$ exists. In particular, we can choose $D$ such that $D \subset \mathcal{A}\left(\mathbf{G}^{0}, \mathbf{T}, k\right)$ for some $E$-split maximal $k$-torus $\mathbf{T}$ in $\mathbf{G}^{0}$.

Fix $\epsilon>0$ such that $\mathfrak{g}_{\epsilon}=\mathfrak{g}_{0^{+}}$. Choose a decreasing sequence $\epsilon_{j}, j=1,2, \cdots$, such that $\epsilon>\epsilon_{j}>0$ and $\epsilon_{j} \rightarrow 0$.

For each $\epsilon_{j}$, there is a strongly good $G$-datum of positive depth $\Sigma_{x_{j}}=\left(\overrightarrow{\mathbf{G}}, x_{j}, \vec{r}, \vec{\phi}\right)$ with $x_{j} \in D$ and $n_{j} \in \mathfrak{g}_{x_{j},-\epsilon_{j}}^{0} \cap \mathcal{N}^{0}$ such that $\left(L_{x_{j}, \epsilon_{j}}, \chi_{\Gamma+n_{j}}\right)<\left(\pi, V_{\pi}\right)$ (such an $x_{j}$ exists by conjugation if necessary). Since $D$ is compact, there is a cluster point $y \in D$ of $x_{j}$ 's.

Let $\epsilon^{\prime}>0$ be such that $\mathfrak{g}_{y, 0^{+}}=\mathfrak{g}_{y, \alpha}=\mathfrak{g}_{y, \alpha^{+}}$and $\mathfrak{g}_{y, s_{i}^{+}}=\mathfrak{g}_{y, s_{i}+\alpha}$ for any $\alpha<\epsilon^{\prime}$. Let $\delta$ be such that for any affine root $\psi$ in $\Psi(\mathbf{G}, \mathbf{T}, E)$ and $x, x^{\prime} \in \mathcal{A}(\mathbf{G}, \mathbf{T}, E)$, if 
$\operatorname{dist}\left(x, x^{\prime}\right)<\delta,\left|\psi\left(x-x^{\prime}\right)\right|<\epsilon^{\prime} / 3$. Then, in that case, for any $r \in \mathbb{R}$,

$$
\mathfrak{g}(E)_{x, r+\epsilon^{\prime} / 3} \stackrel{(\dagger)}{\subset} \mathfrak{g}(E)_{x^{\prime}, r} \subset \mathfrak{g}(E)_{x, r-\epsilon^{\prime} / 3} .
$$

Since $y$ is a cluster point, there are $\epsilon_{j}$ and $x_{j}$ such that $\left|y-x_{j}\right|<\delta$ and $\epsilon_{j}<\epsilon^{\prime} / 3$. Fix such $\epsilon_{j}$ and $x_{j}$. Then we have the following:

(i) With $r=\epsilon_{j}, x=y$ and $x^{\prime}=x_{j}$ in (†), $\mathfrak{g}_{y, 0^{+}}=\mathfrak{g}_{y, \epsilon_{j}+\epsilon^{\prime} / 3} \subset \mathfrak{g}_{x_{j}, \epsilon_{j}}$.

(ii) With $r=\epsilon_{j}+\epsilon^{\prime} / 3, \mathfrak{g}_{y, \epsilon_{j}+2 / 3 \epsilon^{\prime}} \subset \mathfrak{g}_{x_{j}, \epsilon_{j}+\epsilon^{\prime} / 3}$. Since $\mathfrak{g}_{x_{j}, \epsilon_{j}+\epsilon^{\prime} / 3} \subset \mathfrak{g}_{x_{j}, \epsilon_{j}^{+}}$and $\mathfrak{g}_{y, \epsilon_{j}+2 / 3 \epsilon^{\prime}}=\mathfrak{g}_{y, 0^{+}}$, we have $\mathfrak{g}_{y, 0^{+}} \subset \mathfrak{g}_{x_{j}, \epsilon_{j}^{+}}$and $\mathfrak{g}_{x_{j},-\epsilon_{j}} \subset \mathfrak{g}_{y, 0}$.

(iii) With $r=s_{i}+\epsilon^{\prime} / 3, \mathfrak{g}_{y, s_{i}^{+}}=\mathfrak{g}_{y, s_{i}+2 \epsilon^{\prime} / 3} \subset \mathfrak{g}_{x_{j}, s_{i}+\epsilon^{\prime} / 3} \subset \mathfrak{g}_{x_{j}, s_{i}^{+}}$.

From (i)-(iii), we see that $K_{\Sigma_{y}}^{+}=\vec{G}_{y, \vec{s}^{+}} \subset L_{x_{j}, \epsilon_{j}}=\vec{G}_{x_{j}, \vec{s}^{+}\left(\epsilon_{j}\right)}$ and $n_{j} \in \mathfrak{g}_{x_{j},-\epsilon_{j}}^{0} \subset$ $\mathfrak{g}_{y, 0}^{0}$. Hence,

$$
\chi_{\Gamma+n_{j}} \mid \vec{G}_{y, \vec{s}^{+}}=\chi_{\Gamma}
$$

and $\left(\pi, V_{\pi}\right)$ contains $\left(K_{\Sigma_{y}}^{+}, \chi_{\Gamma}\right)$.

Corollary 11.5. Let $\left(\pi, V_{\pi}\right)$ be an irreducible supercuspidal representation. Then, there is a strongly good $G$-datum $\Sigma$ such that $\left(\pi, V_{\pi}\right)$ contains $\left(K_{\Sigma}^{+}, \phi_{\Sigma}\right)$.

Proof. Suppose $\left(\pi, V_{\pi}\right)$ is tempered. Then $\mathfrak{t}:=(G, \pi) \in \mathfrak{B}_{t}$. For any $\Gamma \in \mathfrak{S}$ and $\epsilon>0$ such that $\mathfrak{g}_{0^{+}}=\mathfrak{g}_{\epsilon}$, we have either $\mathcal{E}^{t}(\mathfrak{t}) \subset \mathcal{E}_{\Gamma}^{t}(\epsilon)$ or $\mathcal{E}^{t}(\mathfrak{t}) \cap \mathcal{E}_{\Gamma}^{t}(\epsilon)=\emptyset$. Now, since $\varepsilon^{t}(\mathfrak{t})$ has strictly positive Plancherel measure, the corollary follows from Theorem 11.4 Now, any supercuspidal representation is tempered up to twisting by an unramified character, and the general case follows from the tempered case.

\section{Review of Yu's CONSTRUCTION}

The construction of supercuspidal representations by $\mathrm{Yu}$ is based on a generic $G$-datum (see 38 for details and 3 for a summary).

Definition 12.1. A generic $G$-datum is a quintuple $\Sigma_{Y}=(\overrightarrow{\mathbf{G}}, y, \vec{r}, \vec{\phi}, \rho)$ satisfying the following:

D1. $\overrightarrow{\mathbf{G}}=\left(\mathbf{G}^{0}, \mathbf{G}^{1}, \cdots, \mathbf{G}^{d}=\mathbf{G}\right)$ is a tamely ramified twisted Levi sequence such that $\mathbf{Z}_{\mathbf{G}^{0}} / \mathbf{Z}_{\mathbf{G}}$ is anisotropic.

D2. $y \in \mathcal{B}\left(\mathbf{G}^{0}, k\right)$.

D3. $\vec{r}=\left(r_{0}, r_{1}, \cdots, r_{d-1}, r_{d}\right)$ is a sequence of positive real numbers with $0<r_{0}<$ $\cdots<r_{d-2}<r_{d-1} \leq r_{d}$ if $d>0,0 \leq r_{0}$ if $d=0$.

D4. $\vec{\phi}=\left(\phi_{0}, \cdots, \phi_{d}\right)$ is a sequence of quasi-characters, where $\phi_{i}$ is a generic quasicharacter of $G^{i} ; \phi_{i}$ is trivial on $G_{y, r_{i}^{+}}^{i}$ but nontrivial on $G_{y, r_{i}}^{i}$ for $0 \leq i \leq d-1$. If $r_{d-1}<r_{d}, \phi_{d}$ is nontrivial on $G_{y, r_{d}}^{d}$ and trivial on $G_{y, r_{d}^{+}}^{d}$. Otherwise, $\phi_{d}=1$.

D5. $\rho$ is an irreducible representation of $G_{[y]}^{0}$, the stabilizer in $G^{0}$ of the image [y] of $y$ in the reduced building of $\mathbf{G}^{0}$, such that $\rho \mid G_{y, 0^{+}}^{0}$ is isotrivial and $c$ - $\operatorname{Ind}_{G_{[y]}^{0}}^{G^{0}} \rho$ is irreducible and supercuspidal.

Remark 12.2. By (6.6) and (6.8) of [31], D5 is equivalent to the condition that $G_{y, 0}^{0}$ is a maximal parahoric subgroup in $G^{0}$ and $\rho \mid G_{y, 0}^{0}$ induces a cuspidal representation of $G_{y, 0}^{0} / G_{y, 0^{+}}^{0}$.

As remarked before, under our hypotheses, a generic quasi-character is also good. Instead of reviewing the definition of genericity, we will briefly describe the construction when $\phi_{i}$ is good. 
12.3. From now on, we fix a generic $G$-datum $\Sigma_{Y}=(\overrightarrow{\mathbf{G}}, y, \vec{r}, \vec{\phi}, \rho)$. Associated to $\Sigma_{Y}$, we have the following open compact subgroups. Note that their definitions depend only on $\overrightarrow{\mathbf{G}}, y$ and $\vec{r}$.

(1) $K^{0}:=G_{[y]}^{0} ; K^{0+}:=G_{y, 0^{+}}^{0}$.

(2) $K^{i}:=G_{[y]}^{0} G_{y, s_{0}}^{1} \cdots G_{y, s_{i-1}}^{i} ; K^{i+}:=G_{y, 0^{+}}^{0} G_{y, s_{0}^{+}}^{1} \cdots G_{y, s_{i-1}^{+}}^{i}$ for $1 \leq i \leq d$.

Then note that $K^{i+}=K_{\Sigma}^{i+}$ (see $\left.\$ 5.8\right)$.

(3) $\left.J^{i}:=\left(\mathbf{G}^{i-1}, \mathbf{G}^{i}\right)(k)_{y,\left(r_{i-1}, s_{i-1}\right)} ; J_{+}^{i}:=\left(\mathbf{G}^{i-1}, \mathbf{G}^{i}\right)(k)_{y,\left(r_{i-1}, s_{i-1}^{+}\right.}\right)$.

(4) For $i>0$, we have $K^{i-1} J^{i}=K^{i}$ and $K^{i-1+} J_{+}^{i}=K^{i+}$.

12.4. Construction. Let $\hat{\phi}_{i}$ denote a character of $K^{0} G_{y, 0}^{i} G_{y, s_{i}^{+}}$defined similarly as in $\$ 5.8$ (replacing $G_{y, 0^{+}}^{0}$ with $K^{0} G_{y, 0}^{i}$ ). For $0 \leq i<d$, there exists a representation $\tilde{\phi}_{i}$ of $K^{i} \ltimes J^{i+1}$ such that $\tilde{\phi}_{i} \mid J^{i+1}$ is $\hat{\phi}_{i} \mid J_{+}^{i+1}$-isotypical and $\tilde{\phi}_{i} \mid K^{i+}$ is isotrivial.

Let $\inf \left(\phi_{i}\right)$ denote the inflation of $\phi_{i} \mid K^{i}$ to $K^{i} \ltimes J^{i+1}$. Then $\inf \left(\phi_{i}\right) \otimes \tilde{\phi}_{i}$ factors through a map

$$
K^{i} \ltimes J^{i+1} \longrightarrow K^{i} J^{i+1}=K^{i+1} .
$$

Let $\kappa_{i}$ denote the corresponding representation of $K^{i+1}$. Then it can be extended trivially to $K^{d}$, and we denote the extended representation again by $\kappa_{i}$. We also extend $\rho$ trivially to a representation of $K^{d}$ and denote this extended representation again by $\rho$. Define a representation $\kappa$ and $\rho_{\Sigma_{Y}}$ of $K^{d}$ as follows:

$$
\begin{array}{ll}
\kappa & :=\kappa_{0} \otimes \cdots \otimes \kappa_{d-1} \otimes\left(\phi_{d} \mid K^{d}\right), \\
\rho_{\Sigma_{Y}} & :=\rho \otimes \kappa .
\end{array}
$$

Note that $\kappa$ is defined only from $(\overrightarrow{\mathbf{G}}, y, \vec{r}, \vec{\phi})$ independent of $\rho$.

Theorem $12.5([38]) \cdot \pi_{\Sigma_{Y}}=c-\operatorname{Ind}_{K^{d}}^{G} \rho_{\Sigma_{Y}}$ is an irreducible supercuspidal representation.

Remark 12.6. In $\Sigma_{Y}$, if $d=0$ and $r_{0}=0, \pi_{\Sigma_{Y}}=c$-Ind $\operatorname{I}_{[y]}^{G} \rho$ is a depth zero supercuspial representation. Moreover, by [28] or [31, (6.6), (6.8)], any depth zero supercuspidal representation is of this form. Hence, Yu's construction includes all depth zero supercuspidal representations.

Remark 12.7. Suppose $(\mathrm{H} k),(\mathrm{HB}),(\mathrm{HGT})$ and $(\mathrm{H} \mathcal{N})$ are valid. Let $\left(\pi, V_{\pi}\right)$ be a supercuspidal representation of positive depth. Then by Corollary 11.5, there is a strongly good $G$-datum $\Sigma$ of positive depth so that $\left(K_{\Sigma}^{+}, \phi_{\Sigma}\right)<\left(\pi, V_{\pi}\right)$. Comparing $\Sigma$ and $\Sigma_{Y}$, we note that $\mathrm{Yu}$ imposed certain sufficient conditions on $\Sigma_{Y}$ to get supercuspidal representations such as $(i) \mathbf{Z}_{\mathbf{G}^{0}} / \mathbf{Z}_{\mathbf{G}}$ is anisotropic, (ii) $G_{y, 0}^{0}$ is a maximal parahoric subgroup in $G^{0}$, and (iii) $\rho$ is irreducible and induces a cuspidal representation of $G_{y, 0}^{0} / G_{y, 0^{+}}^{0}$. Now, we will prove that those are also necessary conditions $(\S \S 13-17)$. We start with some preparation.

\section{Preparation}

To prove that the condition $(i)$ in Remark 12.7 is also a necessary condition for supercuspidality (see Proposition [14.5), we will use some basic properties of Hecke algebras. In this section, we review Hecke algebras and deduce various results regarding them (in particular, Corollary 13.12 and Proposition 13.14). 
13.1. Hecke algebras. Let $J$ be an open compact subgroup of $G$ and let $\left(\sigma, V_{\sigma}\right)$ be an irreducible representation of $J$. Denote the contragredient of $\sigma$ by $\tilde{\sigma}$. Then, we can associate a Hecke algebra $\mathcal{H}(G / / J, \sigma)$ to $(J, \sigma)$ as follows:

$\mathcal{H}(G / / J, \sigma)=\left\{f \in C_{c}^{\infty}\left(G, \operatorname{End}_{\mathbb{C}}\left(V_{\tilde{\sigma}}\right)\right) \mid f\left(j g j^{\prime}\right)=\tilde{\sigma}(j) f(g) \tilde{\sigma}\left(j^{\prime}\right)\right.$, for $\left.j, j^{\prime} \in J, g \in G\right\}$.

This is an algebra under convolution with the identity $\frac{1}{\operatorname{vol}(J)} 1_{\tilde{\sigma}} \cdot[J]$. Here, $1_{\tilde{\sigma}}$ is the identity map on $V_{\tilde{\sigma}}$. It is well known that there is a one-to-one correspondence between the set of simple nondegenerate modules of $\mathcal{H}(G / / J, \sigma)$ and $\mathcal{E}_{(J, \sigma)}$. For more details, see for example [5, 8, 22].

We say $g \in G$ is in the support of $\mathcal{H}(G / / J, \sigma)$ if there is an $f \in \mathcal{H}(G / / J, \sigma)$ such that $g$ is in the support of $f$. Denote the set of all such $g$ as $\operatorname{Supp}(\mathcal{H}(G / / J, \sigma))$.

\section{The CASe $\overrightarrow{\mathbf{G}}=\left(\mathbf{G}^{\prime}, \mathbf{G}\right)$}

13.2. Let $\overrightarrow{\mathbf{G}}=\left(\mathbf{G}^{\prime}, \mathbf{G}\right)$ be a tamely ramified twisted Levi sequence, and let $y \in$ $\mathcal{B}\left(\mathbf{G}^{\prime}, k\right)$. Let $\phi$ be a $G$-good character of $G^{\prime}$ such that on $G_{y, r}^{\prime}$, it is represented by a $G$-good element $\gamma \in \mathfrak{z}_{\mathfrak{g}^{\prime}}$ of depth $-r<0$ with $\mathbf{C}_{\mathbf{G}}(\gamma)=\mathbf{G}^{\prime}$. Set $s:=\frac{r}{2}$.

Let $\mathbf{T} \subset \mathbf{G}^{\prime}$ be an $E$-split maximal $k$-torus such that $y \in \mathcal{A}(\mathbf{G}, \mathbf{T}, k)$. Let $\mathbf{S}$ be a $k$-split subtorus in $\mathbf{T}$. Let $\mathbf{M}$ be the $k$-Levi subgroup of $\mathbf{G}$ defined as $\mathbf{C}_{\mathbf{G}}(\mathbf{S})$. Since $\mathbf{T} \subset \mathbf{M}, y \in \mathcal{B}(\mathbf{M}, k)$. Let $\mathbf{P}$ be a $k$-parabolic subgroup with Levi decomposition $\mathbf{P}=\mathbf{M U}$, and let $\overline{\mathbf{U}}$ be the unipotent subgroup opposite to $\mathbf{U}$. Define $f_{t}: \Phi(\mathbf{G}, \mathbf{T}, E) \cup\{0\} \longrightarrow \tilde{\mathbb{R}}$ and $f_{t}^{\prime}: \Phi\left(\mathbf{G}^{\prime}, \mathbf{T}, E\right) \cup\{0\} \longrightarrow \tilde{\mathbb{R}}$ as

$$
f_{t}(a)= \begin{cases}t^{+} & \text {if } a \in \Phi(\mathbf{M}, \mathbf{T}, E) \cup\{0\}, \\ t^{+} & \text {if } a \in \Phi(\overline{\mathbf{U}}, \mathbf{T}, E), \\ t & \text { if } a \in \Phi(\mathbf{U}, \mathbf{T}, E)\end{cases}
$$

and

$$
f_{t}^{\prime}(a)= \begin{cases}t^{+} & \text {if } a \in \Phi\left(\mathbf{M} \cap \mathbf{G}^{\prime}, \mathbf{T}, E\right) \cup\{0\}, \\ t^{+} & \text {if } a \in \Phi\left(\overline{\mathbf{U}} \cap \mathbf{G}^{\prime}, \mathbf{T}, E\right), \\ t & \text { if } a \in \Phi\left(\mathbf{U} \cap \mathbf{G}^{\prime}, \mathbf{T}, E\right)\end{cases}
$$

and

$$
\tilde{f}_{t}(a)= \begin{cases}t & \text { if } a \in \Phi(\mathbf{M}, \mathbf{T}, E) \cup\{0\} \\ t^{+} & \text {if } a \in \Phi(\overline{\mathbf{U}}, \mathbf{T}, E), \\ t & \text { if } a \in \Phi(\mathbf{U}, \mathbf{T}, E) .\end{cases}
$$

Here and later, $\Phi(?, \mathbf{T}, E)$ denotes the set of $E$-roots of $\mathbf{T}$ in ?. To avoid any confusion, it should be pointed out that the above $\tilde{f}_{t}$ is totally unrelated to $\tilde{\sigma}$ in \$13.1.

Note that $f_{t}^{*}=\tilde{f}_{-t}$. We write

$$
\bar{U}_{y, t}=\bar{U} \cap G_{y, t}, \quad \overline{\mathfrak{u}}_{y, t}=\overline{\mathfrak{u}} \cap \mathfrak{g}_{y, t}, \quad \bar{U}_{y, t}^{\prime}=\bar{U} \cap G_{y, t}^{\prime}, \quad \overline{\mathfrak{u}}_{y, t}^{\prime}=\overline{\mathfrak{u}} \cap \mathfrak{g}_{y, t}^{\prime} .
$$

Recall that we have an orthogonal decomposition $\mathfrak{g}=\mathfrak{g}^{\prime} \oplus \mathfrak{g}^{\perp}$ with respect to $\mathrm{B}$ when $(\mathrm{HB})$ is valid. Otherwise, we can take $\mathfrak{g}^{\perp}=[\gamma, \mathfrak{g}]$ to have a linear decomposition of $\mathfrak{g}$. In both case, $\mathfrak{g}^{\perp}$ is invariant under ad $(\gamma)$. Let $\mathfrak{g}_{y, \tilde{f}_{t}}^{\perp}:=\mathfrak{g}_{y, \tilde{f}_{t}} \cap$ $\mathfrak{g}^{\perp}$.

Lemma 13.3. Let $Y^{\prime} \in \mathfrak{g}_{y,(-r)^{+}}^{\prime}$, and let $t \in \tilde{\mathbb{R}}$.

(1) Let $t \in \mathbb{R}$. Then, $\operatorname{ad}\left(\gamma+Y^{\prime}\right): \mathfrak{g}_{y, \tilde{f}_{t}}^{\perp} / \mathfrak{g}_{y, \tilde{f}_{t+1}}^{\perp} \longrightarrow \mathfrak{g}_{y, \tilde{f}_{t-r}}^{\perp} / \mathfrak{g}_{y, \tilde{f}_{t-r+1}}^{\perp} \quad$ is an isomorphism.

(2) Let $t \in \mathbb{R}$ with $t>-r$. Then, ${ }^{G_{y, \tilde{f}_{r+t}}}\left(\gamma+\mathfrak{g}_{y, \tilde{f}_{t}} \cap \mathfrak{g}^{\prime}\right)=\gamma+\mathfrak{g}_{y, \tilde{f}_{t}}$. 
(3) If $Y^{\prime} \in \mathfrak{g}_{y,(-r)^{+}}^{\prime} \cap \overline{\mathfrak{u}}$, then $\operatorname{ad}\left(\gamma+Y^{\prime}\right):\left(\mathfrak{g}_{y, t}^{\prime \perp} \cap \overline{\mathfrak{u}}\right) /\left(\mathfrak{g}_{y, t+1}^{\prime \perp} \cap \overline{\mathfrak{u}}\right) \longrightarrow$ $\left(\mathfrak{g}_{y, t-r}^{\prime \perp} \cap \overline{\mathfrak{u}}\right) /\left(\mathfrak{g}_{y, t-r+1}^{\prime} \cap \bar{\perp} \overline{\mathfrak{u}}\right)$ is an isomorphism.

(4) Let $Y^{\prime} \in \mathfrak{g}_{y,(-r)+}^{\prime} \cap \overline{\mathfrak{u}}$. Suppose $t>-r$. Then $\bar{U}_{y, r+t}\left(\gamma+Y^{\prime}+\overline{\mathfrak{u}}_{y, t}^{\prime}\right)=$ $\gamma+Y^{\prime}+\overline{\mathfrak{u}}_{y, t}$.

(5) Let $u \in \bar{U}_{y, s}$. Then, ${ }^{u}\left(\gamma+\overline{\mathfrak{u}}_{y,(-s)^{+}}\right)=\left(\gamma+\overline{\mathfrak{u}}_{y,(-s)^{+}}\right)$if and only if $u \in$ $\bar{U}_{y, s} \bar{U}_{y, s}^{\prime}=\bar{U}_{y, s}^{\prime} \bar{U}_{y, s^{+}}$.

Proof. (1) Suppose $Y^{\prime}=0$. Since $\tilde{f}_{t}$ is Galois invariant, we have $\mathfrak{g}(E)_{y, \tilde{f}_{t}} \cap \mathfrak{g}=$ $\mathfrak{g}_{y, \tilde{f}_{t}}([3])$. Moreover, from the definition of $\tilde{f}_{t}$ and the properties of $\mathrm{B}$, we have $\mathfrak{g}(E)_{y, \tilde{f}_{t}} \cap \mathfrak{g}^{\prime}(E)^{\perp} \cap \mathfrak{g}=\mathfrak{g}_{y, \tilde{f}_{t}}^{\perp}$ where $\mathfrak{g}(E)=\mathfrak{g}^{\prime}(E) \oplus \mathfrak{g}^{\prime}(E)^{\perp}$. Hence we may assume that $\gamma$ is $k$-split. Then (1) results from $\gamma$ being good. If $Y^{\prime} \neq 0, \operatorname{since} \operatorname{ad}(\gamma)$ is an isomorphism and $\operatorname{ad}\left(Y^{\prime}\right)$ acts pronilpotently, $\operatorname{ad}\left(\gamma+Y^{\prime}\right)$ is also an isomorphism (see also [26, (2.3.4)]).

(2) Having (1), (2) follows from the usual approximation argument (see 4, (6.3)] or [38, (8.5)-(8.6)]). We omit the details.

(3) Since $\operatorname{ad}\left(\gamma+Y^{\prime}\right)(\overline{\mathfrak{u}}) \subset \overline{\mathfrak{u}}$, (3) follows from (1).

(4) Using (3), one can use an approximation argument.

(5) Since ${ }^{u} \overline{\mathfrak{u}}_{y,(-s)^{+}}=\overline{\mathfrak{u}}_{y,(-s)^{+}},{ }^{u}\left(\gamma+\overline{\mathfrak{u}}_{y,(-s)^{+}}\right)=\left(\gamma+\overline{\mathfrak{u}}_{y,(-s)^{+}}\right)$if and only if ${ }^{u} \gamma-\gamma \in \overline{\mathfrak{u}}_{y,(-s)^{+}}$. We claim ${ }^{u} \gamma-\gamma \in \overline{\mathfrak{u}}_{y,(-s)^{+}}$if and only if $u \in \bar{U}_{y, s^{+}} \bar{U}_{y, s}^{\prime}$. Suppose $u \in \bar{U}_{y, s^{+}} \bar{U}_{y, s}^{\prime}$. Then, ${ }^{u} \gamma \in \bar{U}_{y, s^{+}}\left(\gamma+\overline{\mathfrak{u}}_{y,(-s)^{+}}^{\prime}\right)=\gamma+\overline{\mathfrak{u}}_{y,(-s)^{+}}$by $(4)$, and ' $\Leftarrow$ ' follows. Conversely, suppose ${ }^{u} \gamma \in \gamma+\overline{\mathfrak{u}}_{y,(-s)^{+}}$. Then by (4), there are $u_{1} \in \bar{U}_{y, s^{+}}$and $X^{\prime} \in \overline{\mathfrak{u}}_{y,(-s)^{+}}^{\prime}$ such that ${ }^{u} \gamma={ }^{u_{1}}\left(\gamma+X^{\prime}\right)$. Since $\gamma$ is good and $X^{\prime} \in \mathfrak{g}_{y,(-r)^{+}}^{\prime}, u^{-1} u_{1} \in\left(G^{\prime} \cap \bar{U}_{y, s}\right)$ by Lemma 4.5(1). Hence, $u \in \bar{U}_{y, s^{+}} \bar{U}_{y, s}^{\prime}$. Now, (5) follows from the claim.

13.4. Define

$$
K_{\vdash}^{0}:=G_{y, f_{0}^{\prime}}^{\prime}, \quad K_{\vdash}^{1}:=G_{y, f_{s}}, \quad K_{\vdash}:=K_{\vdash}^{0} K_{\vdash}^{1} \quad \text { and } \quad \tilde{K}_{\vdash}:=G_{y, \tilde{f}_{s}} .
$$

Suppose $\phi$ is represented on $G_{y, s^{+}}$again by $\gamma$. Then, by [9, (6.4.44)], we have $\left[K_{\vdash}^{1}, K_{\vdash}^{1}\right] \subset \vec{G}_{y,\left(r^{+}, s^{+}\right)} \subset \operatorname{ker}(\phi)$. Hence, there is a unique character of $K_{\vdash}^{1}$ with dual blob $\gamma+\mathfrak{g}_{y, f_{s}^{*}}=\gamma+\mathfrak{g}_{y, \tilde{f}_{-s}}$. As before, we denote this character by $\chi_{\gamma}$.

Lemma 13.5. Assume $(\mathrm{HB})$ is valid. Suppose $Y^{\prime} \in \mathfrak{g}_{y,(-r)^{+}}^{\prime}$ and $\gamma+Y^{\prime}+\mathfrak{g}_{y, f_{s}^{*}}$ represent a character of $K_{\vdash}^{1}$. Then, the support of $\mathcal{H}=\mathcal{H}\left(G / / K_{\vdash}^{1}, \chi_{\gamma+Y^{\prime}}\right)$ is contained in $\tilde{K}_{\vdash} G^{\prime} \tilde{K}_{\vdash}$.

Proof. Note that $\tilde{\chi}_{\gamma+Y^{\prime}}=\chi_{-\gamma-Y^{\prime}}$. Suppose $g \in G$ is in the support of $\mathcal{H}$. Then, ${ }^{g} \chi_{-\gamma-Y^{\prime}}=\chi_{-\gamma-Y^{\prime}}$ on ${ }^{g} K_{\vdash}^{1} \cap K_{\vdash}^{1}$. By Lemma 3.12, we have ${ }^{g}\left(-\gamma-Y^{\prime}+\mathfrak{g}_{y, f_{s}^{*}}\right) \cap$ $\left(-\gamma-Y^{\prime}+\mathfrak{g}_{y, f_{s}^{*}}\right) \neq \emptyset$, and thus ${ }^{g}\left(\gamma+Y^{\prime}+\mathfrak{g}_{y, f_{s}^{*}}\right) \cap\left(\gamma+Y^{\prime}+\mathfrak{g}_{y, f_{s}^{*}}\right) \neq \emptyset$. By Lemma 13.3 (2), there are $j, j^{\prime} \in \tilde{K}_{\vdash}$ and $X_{1}^{\prime}, X_{2}^{\prime} \in \mathfrak{g}^{\prime} \cap \mathfrak{g}_{y, f_{s}^{*}}$ such that ${ }^{g j^{\prime}}\left(\gamma+Y^{\prime}+X_{1}^{\prime}\right)=$ ${ }^{j}\left(\gamma+Y^{\prime}+X_{2}^{\prime}\right)$. Since $\gamma+Y^{\prime}+X_{1}^{\prime}, \gamma+Y^{\prime}+X_{2}^{\prime} \in \gamma+\mathfrak{g}_{(-r)^{+}}^{\prime}$, we have $j^{-1} g j^{\prime} \in G^{\prime}$ by Lemma 4.5 (1). Hence $g \in \tilde{K}_{\vdash} G^{\prime} \tilde{K}_{\vdash}$.

Remark 13.6. All the statements in Lemmas 13.3 and 13.5 are also valid when we replace $\gamma$ with $\gamma+Z$ for $Z \in \mathfrak{z} \mathfrak{g}$.

13.7. Now, suppose that $\phi^{\prime}$ is a character of $G^{\prime}$ so that $\phi^{\prime} \mid G_{y, 0^{+}}^{\prime}$ is represented by a $G$-good element $\gamma^{\prime} \in \mathfrak{z}_{\mathfrak{g}^{\prime}}$ of depth $-r$. However, we do not assume that 
$\mathbf{C}_{\mathbf{G}}\left(\gamma^{\prime}\right)=\mathbf{G}^{\prime}$. Note that $\gamma^{\prime}$ still defines a character $\chi_{\gamma^{\prime}}$ on $K_{\vdash}$ such that $\chi_{\gamma^{\prime}} \mid U_{y, 0}^{\prime}$ and $\chi_{\gamma^{\prime}} \mid \bar{U}_{y, 0^{+}}^{\prime}$ are trivial. The following lemma will be used for the proof of Proposition 13.11

Lemma 13.8. Suppose (HB) and $(\mathrm{H} k)$ are valid. Let $\vec{s}=(0, s)$ and $\vec{s}^{+}=\left(0^{+}, s^{+}\right)$. Let $u \in \bar{U}_{y, s}$. Let $\gamma^{\prime}$ and $\chi_{\gamma^{\prime}}$ be as above. Then ${ }^{u} \chi_{\gamma^{\prime}} \equiv \chi_{\gamma^{\prime}}$ on $\vec{G}_{y, \vec{s}^{+}}$and ${ }^{u} \chi_{\gamma^{\prime}} \mid U_{y, 0}^{\prime}=1$.

Proof. Note that $\bar{U}_{y, s}$ normalizes $K_{\vdash}$. Write $\log (u)=\left(X^{\prime}+X^{\perp}\right)$ for $X^{\prime} \in \overline{\mathfrak{u}}_{y, s}^{\prime}$ and $X^{\perp} \in \overline{\mathfrak{u}}_{y, s} \cap \mathfrak{g}^{\perp}$. Then, by $(\mathrm{H} k)$, we have

$$
\operatorname{Ad}(u)\left(\gamma^{\prime}\right)=\sum_{n=1}^{\infty} \frac{1}{n !}\left(\operatorname{ad}\left(X^{\prime}+X^{\perp}\right)\right)^{n}\left(\gamma^{\prime}\right) .
$$

Since $\left[\gamma^{\prime}, \mathfrak{g}^{\prime}\right]=0, \quad\left[\gamma^{\prime}, \mathfrak{g}^{\perp} \cap \overline{\mathfrak{u}}\right] \subset \mathfrak{g}^{\perp} \cap \overline{\mathfrak{u}}, \quad\left[\mathfrak{g}^{\perp} \cap \overline{\mathfrak{u}}, \mathfrak{g}^{\prime \perp} \cap \overline{\mathfrak{u}}\right] \subset \mathfrak{g}^{\perp} \cap \overline{\mathfrak{u}}$ and $\quad\left[\mathfrak{g}^{\prime} \cap\right.$ $\left.\overline{\mathfrak{u}}, \mathfrak{g}^{\perp} \cap \overline{\mathfrak{u}}\right] \subset \mathfrak{g}^{\prime \perp} \cap \overline{\mathfrak{u}}$, from the above formula and (Hk), we conclude ${ }^{u} \gamma^{\prime}-\gamma^{\prime} \in$ $\left(\overline{\mathfrak{u}}_{y,-s} \cap \mathfrak{g}^{\perp}\right) \subset\left(\overrightarrow{\mathfrak{g}}_{y,-\vec{s}} \cap \mathfrak{g}^{\perp \perp}\right)=\left(\overrightarrow{\mathfrak{g}}_{y,-\vec{s}} \cap \mathfrak{g}^{\perp}\right)$. Then ${ }^{u} \gamma^{\prime}-\gamma^{\prime} \in \mathfrak{g}^{\perp}$ implies that $1=\chi_{\gamma^{\prime}}\left|U_{y, 0}^{\prime}={ }^{u} \chi_{\gamma^{\prime}}\right| U_{y, 0}^{\prime}$, and ${ }^{u} \gamma^{\prime}-\gamma^{\prime} \in \overrightarrow{\mathfrak{g}}_{y,-\vec{s}}=\left(\overrightarrow{\mathfrak{g}}_{y, \vec{s}^{+}}\right)^{*}$ implies that ${ }^{u} \chi_{\gamma^{\prime}} \mid \vec{G}_{y, \vec{s}^{+}}=$ $\chi_{\gamma^{\prime}} \mid \vec{G}_{y, \vec{s}^{+}}$. Hence the lemma follows.

\section{General CASES}

13.9. Let $\Sigma_{y}=(\overrightarrow{\mathbf{G}}, y, \vec{r}, \vec{\phi})$ be a good $G$-datum of positive depth. Let $\mathbf{T} \subset \mathbf{G}^{0}$ be an $E$-split maximal $k$-torus such that $y \in \mathcal{A}(\mathbf{G}, \mathbf{T}, k)$. Let $\mathbf{S}$ be a $k$-split subtorus in $\mathbf{T}$. Let $\mathbf{M}$ be the $k$-Levi subgroup of $\mathbf{G}$ defined by $\mathbf{C}_{\mathbf{G}}(\mathbf{S})$. Then, $y \in \mathcal{B}(\mathbf{M}, k)$. Fix a $k$-parabolic subgroup $\mathbf{P}$ with Levi decomposition $\mathbf{P}=\mathbf{M U}$, and let $\overline{\mathbf{U}}$ be the unipotent subgroup opposite to $\mathbf{U}$. Let

$$
\mathbf{M}^{i}:=\mathbf{M} \cap \mathbf{G}^{i}=\mathbf{C}_{\mathbf{G}^{i}}(\mathbf{S}), \quad \mathbf{U}^{i}:=\mathbf{U} \cap \mathbf{G}^{i}, \quad \overline{\mathbf{U}}^{i}=\overline{\mathbf{U}} \cap \mathbf{G}^{i}
$$

for $i=0,1, \cdots, d$. Note that each $\mathbf{M}^{i}$ is a $k$-Levi subgroup of $\mathbf{G}^{i}$.

Define $f^{i}: \Phi\left(\mathbf{G}^{i}, \mathbf{T}, E\right) \cup\{0\} \longrightarrow \tilde{\mathbb{R}}$ as follows: if $i \geq 1$,

$$
f^{i}(a)= \begin{cases}s_{i-1}^{+} & \text {if } a \in \Phi\left(\mathbf{M}^{i}, \mathbf{T}, E\right) \cup\{0\}, \\ s_{i-1}^{+} & \text {if } a \in \Phi\left(\overline{\mathbf{U}}^{i}, \mathbf{T}, E\right), \\ s_{i-1} & \text { if } a \in \Phi\left(\mathbf{U}^{i}, \mathbf{T}, E\right)\end{cases}
$$

and

$$
f^{0}(a)= \begin{cases}0^{+} & \text {if } a \in \Phi\left(\mathbf{M}^{0}, \mathbf{T}, E\right) \cup\{0\}, \\ 0^{+} & \text {if } a \in \Phi\left(\overline{\mathbf{U}}^{0}, \mathbf{T}, E\right), \\ 0 & \text { if } a \in \Phi\left(\mathbf{U}^{0}, \mathbf{T}, E\right) .\end{cases}
$$

For $i=1, \cdots, d$, define $\tilde{f}^{i}: \Phi\left(\mathbf{G}^{i}, \mathbf{T}, E\right) \cup\{0\} \longrightarrow \tilde{\mathbb{R}}$ as

$$
\tilde{f}^{i}(a)= \begin{cases}s_{i-1} & \text { if } a \in \Phi\left(\mathbf{M}^{i}, \mathbf{T}, E\right) \cup\{0\}, \\ s_{i-1}^{+} & \text {if } a \in \Phi\left(\overline{\mathbf{U}}^{i}, \mathbf{T}, E\right), \\ s_{i-1} & \text { if } a \in \Phi\left(\mathbf{U}^{i}, \mathbf{T}, E\right) .\end{cases}
$$

We also define corresponding open compact subgroups of $G^{i}$ as follows: for $i=$ $0,1, \cdots, d$,

and

$$
K_{y \vdash}^{i}:=G_{y, f^{i}}^{i} \subset G^{i}
$$

$$
\tilde{K}_{y \vdash}^{0}:=1, \quad \tilde{K}_{y \vdash}^{i}:=G_{y, \tilde{f}^{i}}^{i} \subset G^{i} .
$$


If there is no confusion, we omit $y$ from the above notation and simply write $K_{\vdash}^{i}$ and $\tilde{K}_{\vdash}^{i}$. Note that we have $K_{\vdash}^{0} \subset G^{0}$ and $K_{\vdash}^{0}=G_{y, 0^{+}}^{0}$ if $\mathbf{G}^{0} \subset \mathbf{M}$. Define

$$
K_{y \vdash}=K_{\vdash}:=K_{\vdash}^{0} K_{\vdash}^{1} \cdots K_{\vdash}^{d} \quad \text { and } \quad \tilde{K}_{y \vdash}=\tilde{K}_{\vdash}:=\tilde{K}_{\vdash}^{0} \tilde{K}_{\vdash}^{1} \cdots \tilde{K}_{\vdash}^{d} .
$$

Note that the above open compact subgroups depend also on the choice of $\mathbf{M}$ and U.

If $\Sigma_{y}$ is strongly good, each $\phi_{i}$ defines a character of $K_{\vdash}$ represented by $\Gamma_{i}$ and $\vec{\phi}$ defines a character of $K_{\vdash}$ represented by $\Gamma$. We again denote these characters by $\chi_{\Gamma_{i}}$ and $\chi_{\Gamma}$, respectively.

For $i=0, \cdots, d$ and $t \in \tilde{\mathbb{R}}_{\geq 0}$, we have $M_{y, t}^{i}=G_{y, t}^{i} \cap M$. Write

$$
\bar{U}_{y, t}^{i}:=G_{y, t}^{i} \cap \bar{U}, \quad U_{y, t}^{i}:=G_{y, t}^{i} \cap U .
$$

For any admissible sequence $\vec{t}$ of length $\ell(\Sigma)$, we also write

$$
\bar{U}_{y, \vec{t}}:=\vec{G}_{y, \vec{t}} \cap \bar{U}, \quad M_{y, \vec{t}}:=\vec{G}_{y, \vec{t}} \cap M, \quad U_{y, \vec{t}}:=\vec{G}_{y, \vec{t}} \cap U .
$$

Then note that $U_{y, \vec{s}}=K_{\vdash} \cap U=\tilde{K}_{\vdash} \cap U, \bar{U}_{y, \vec{s}^{+}}=K_{\vdash} \cap \bar{U}=\tilde{K}_{\vdash} \cap \bar{U}, M_{y, \vec{s}^{+}}=$ $K_{\vdash} \cap M$, etc.

Lemma 13.10. For (2) and (3), we assume (Hk) and (HB) are valid.

(1) For $i=0, \cdots, d-1$, the following is an isomorphism:

$$
\operatorname{ad}\left(\Gamma_{i}\right):\left(\overline{\mathfrak{u}}_{y, \vec{s}} \cap \mathfrak{g}^{i \perp}\right) /\left(\overline{\mathfrak{u}}_{y, \vec{s}^{+}} \cap \mathfrak{g}^{i \perp}\right) \longrightarrow\left(\overline{\mathfrak{u}}_{y,-\vec{s}} \cap \mathfrak{g}^{i \perp}\right) /\left(\overline{\mathfrak{u}}_{y,(-\vec{s})}+\cap \mathfrak{g}^{i \perp}\right) .
$$

(2) $\left|\overline{\mathfrak{u}}_{y, \vec{s}} / \overline{\mathfrak{u}}_{y, \vec{s}^{+}}\right|=\left|\overline{\mathfrak{u}}_{y,-\vec{s}} / \overline{\mathfrak{u}}_{y,(-\vec{s})+}\right|=\left|U_{y, \vec{s}} / U_{y, \vec{s}^{+}}\right|=\left|K_{\vdash} / K_{\Sigma}^{+}\right|$.

(3) $\left|\overline{\mathfrak{u}}_{y, \vec{s}\left(0^{+}\right)} / \overline{\mathfrak{u}}_{y, \vec{s}^{+}}\right|=\left|U_{y, \vec{s}\left(0^{+}\right)} / U_{y, \vec{s}^{+}}\right|=\left|K_{\vdash} /\left(U_{y, 0}^{0} K_{\Sigma}^{+}\right)\right|$where $\vec{s}\left(0^{+}\right)=$ $\left(0^{+}, s_{0}, \cdots, s_{d-1}\right)$.

Proof. (1) Since $\Gamma_{i}$ is a $G^{i+1}$-good element of depth $-r_{i}$ and $\operatorname{ad}\left(\Gamma_{i}\right)(\overline{\mathfrak{u}}) \subset(\overline{\mathfrak{u}})$, the above $\operatorname{ad}\left(\Gamma_{i}\right)$ is an isomorphism by [1, (2.3.1)].

(2) Since $\mathfrak{g}=\mathfrak{g}^{0} \oplus \mathfrak{g}^{0 \perp} \oplus \cdots \mathfrak{g}^{d-1 \perp}$, we have

$$
\begin{aligned}
& \left|\overline{\mathfrak{u}}_{y, \vec{s}} / \overline{\mathfrak{u}}_{y, \vec{s}^{+}}\right|=\left|\overline{\mathfrak{u}}_{y, 0}^{0} / \overline{\mathfrak{u}}_{y, 0^{+}}^{0}\right| \cdot \prod_{i=0}^{d-1}\left|\left(\overline{\mathfrak{u}}_{y, \vec{s}} \cap \mathfrak{g}^{i \perp}\right) /\left(\overline{\mathfrak{u}}_{y, \vec{s}^{+}} \cap \mathfrak{g}^{i \perp}\right)\right| \\
& \quad=\left|\overline{\mathfrak{u}}_{y, 0}^{0} / \overline{\mathfrak{u}}_{y, 0^{+}}^{0}\right| \cdot \prod_{i=0}^{d-1}\left|\left(\overline{\mathfrak{u}}_{y,-\vec{s}} \cap \mathfrak{g}^{i \perp}\right) /\left(\overline{\mathfrak{u}}_{y,(-\vec{s})} \cap \cap \mathfrak{g}^{i \perp}\right)\right|=\left|\overline{\mathfrak{u}}_{y,-\vec{s}} / \overline{\mathfrak{u}}_{y,(-\vec{s})^{+}}\right| .
\end{aligned}
$$

The second equality follows from (1). By duality,

$$
\left|\overline{\mathfrak{u}}_{y,-\vec{s}} / \overline{\mathfrak{u}}_{y,(-\vec{s})^{+}}\right|=\left|\left(\mathfrak{u}_{y, \vec{s}^{+}}^{*} \cap \overline{\mathfrak{u}}\right) /\left(\mathfrak{u}_{y, \vec{s}}^{*} \cap \overline{\mathfrak{u}}\right)\right|=\left|\mathfrak{u}_{y, \vec{s}} / \mathfrak{u}_{y, \vec{s}^{+}}\right| .
$$

Since $\log \left(K_{\vdash}\right) \cap \mathfrak{u}=\mathfrak{u}_{y, \vec{s}}, \log \left(K_{\Sigma}^{+}\right) \cap \mathfrak{u}=\mathfrak{u}_{y, \vec{s}^{+}}$, and $\log \left(K_{\vdash}\right) \cap(\overline{\mathfrak{u}} \oplus \mathfrak{m})=\log \left(K_{\Sigma}^{+}\right) \cap$ $(\overline{\mathfrak{u}} \oplus \mathfrak{m})$, we have $\left|U_{y, \vec{s}} / U_{y, \vec{s}^{+}}\right|=\left|\mathfrak{u}_{y, \vec{s}} / \mathfrak{u}_{y, \vec{s}^{+}}\right|=\left|\log \left(K_{\vdash}\right) / \log \left(K_{\Sigma}^{+}\right)\right|=\left|K_{\vdash} / K_{\Sigma}^{+}\right|$.

(3) By duality, we have $\left|\overline{\mathfrak{u}}_{y, 0}^{0} / \overline{\mathfrak{u}}_{y, 0^{+}}^{0}\right|=\left|\mathfrak{u}_{y, 0}^{0} / \mathfrak{u}_{y, 0^{+}}^{0}\right|$. Denote this number by $m$. Dividing each term in (2) by $m$, we get the equalities in (3).

Proposition 13.11. Suppose $\Sigma=\Sigma_{y}$ is a strongly good $G$-datum of positive depth. Suppose $(\mathrm{HB})$ and $(\mathrm{H} k)$ are valid. Let $\chi$ be an irreducible representation of $K_{\vdash}$ such that $\chi \mid K_{\Sigma}^{+}$contains $\chi_{\Gamma}$ and $\chi \mid U_{y, 0}^{0} \equiv 1$. Then there is $a u \in \bar{U}_{y, \vec{s}\left(0^{+}\right)}$such that $\chi \equiv{ }^{u} \chi_{\Gamma}$ on $K_{\vdash}$. 
Proof. Let $u \in \bar{U}_{y, \vec{s}\left(0^{+}\right)}$. Then, we can write $u=u_{d} \cdots u_{1} u_{0}$ for some $u_{0} \in \bar{U}_{y, 0^{+}}^{0}$ and $u_{i} \in \bar{U}_{y, s_{i-1}}^{i}, i=1, \cdots, d$. Then

For the character $\chi_{\Gamma_{j}}$ of $K_{\vdash}$, if $j \geq i$, we have ${ }^{u_{i}} \chi_{\Gamma_{j}} \equiv \chi_{\Gamma_{j}}$ on $K_{\vdash}$ since $u_{i} \in G^{j}$.

$$
u_{d} u_{d-1} \cdots u_{0} \chi_{\Gamma_{i-1}}={ }^{u_{d} u_{d-2} \cdots u_{i}} \chi_{\Gamma_{i-1}}={ }^{u_{i} u_{i}^{-1}\left(u_{d} u_{d-2} \cdots u_{i+1}\right) u_{i}} \chi_{\Gamma_{i-1}}={ }^{u_{i}} \chi_{\Gamma_{i-1}} .
$$

The last equality follows from $u_{i}^{-1}\left(u_{d} u_{d-2} \cdots u_{i+1}\right) u_{i} \in \bar{U}_{y, s_{i}} \subset \operatorname{ker}\left(\chi_{\Gamma_{i-1}} \mid G_{y, s_{i-1}^{+}}\right)$. Hence

$$
u_{d} u_{d-1} \cdots u_{0} \chi_{\Gamma}={ }^{u_{1}} \chi_{\Gamma_{0}}{ }^{u_{2}} \chi_{\Gamma_{1}} \cdots{ }^{u_{d}} \chi_{\Gamma_{d-1}} \chi_{\Gamma_{d}} \text { on } K_{\vdash} .
$$

Since $K_{\Sigma}^{+} \subset\left(\mathbf{G}^{i-1}, \mathbf{G}\right)(k)_{y,\left(0^{+}, s_{i-1}^{+}\right)}$and $U_{y, 0}^{0} \subset U_{y, 0}^{i-1}$, from Lemma 13.8, we deduce that ${ }^{u_{i}} \chi_{\Gamma_{i-1}} \equiv \chi_{\Gamma_{i-1}}$ on $K_{\Sigma}^{+}$and ${ }^{u_{i}} \chi_{\Gamma_{i-1}} \mid U_{y, 0}^{0} \equiv 1$. Hence, for any $u \in \bar{U}_{y, \vec{s}\left(0^{+}\right)}$, we have ${ }^{u} \chi_{\Gamma}\left|K_{\Sigma}^{+}=\chi_{\Gamma}\right| K_{\Sigma}^{+}$and ${ }^{u} \chi_{\Gamma} \mid U_{y, 0}^{0}=1$.

We claim that ${ }^{u} \chi_{\Gamma} \equiv \chi_{\Gamma}$ on $K_{\vdash}$ if and only if $u \in \bar{U}_{y, \vec{s}^{+}}$. Since $u \in \bar{U}_{y, \vec{s}^{+}} \subset K_{\vdash}$, ' $\Leftarrow$ ' is obvious. To prove ' $\Rightarrow$ ', we first observe that ${ }^{u} \chi_{\Gamma}=\chi_{\Gamma_{0}} \chi_{\Gamma_{1}} \cdots{ }^{u_{i}} \chi_{\Gamma_{i-1}} \cdots \chi_{\Gamma_{d}}$ on $G_{y, s_{i-2}^{+}}^{i-1} K_{\vdash}^{i}$. Hence, ${ }^{u} \chi_{\Gamma} \equiv \chi_{\Gamma}$ on $K_{\vdash}$ implies ${ }^{u_{i}} \chi_{\Gamma_{i-1}}=\chi_{\Gamma_{i-1}}$ on $G_{y, s_{i-2}^{+}}^{i-1} K_{\vdash}^{i}$. Then, $u_{i} \in \bar{U}_{y, s_{i-1}^{+}}^{i} \bar{U}_{y, s_{i-1}}^{i-1}$ by Lemma 13.3(5). Hence, $u=u_{d} \cdots u_{0} \in \bar{U}_{y, s_{d-1}^{+}}^{d} \cdots$ $\bar{U}_{y, s_{0}^{+}}^{1} \bar{U}_{y, 0^{+}}^{0}$. The claim is now proved.

From the claim and Lemma 13.10. $\left|\left\{{ }^{u} \chi_{\Gamma} \mid u \in \bar{U}_{y, \vec{s}\left(0^{+}\right)}\right\}\right|=\left|\bar{U}_{y, \vec{s}\left(0^{+}\right)} / \bar{U}_{y, \vec{s}^{+}}\right|=$ $\left|K_{\vdash} /\left(U_{y, 0}^{0} K_{\Sigma}^{+}\right)\right|$. Hence, by counting, any irreducible extension of $\chi_{\Gamma}$ of $K_{\Sigma}^{+}$to $K_{\vdash}$ which is trivial on $U_{y, 0}^{0}$ is of the form ${ }^{u} \chi_{\Gamma}$ for some $u \in \bar{U}_{y, \vec{s}\left(0^{+}\right)}$.

For later use, we record the following corollary:

Corollary 13.12. Suppose $\Sigma=\Sigma_{y}$ is a strongly good $G$-datum of positive depth. Assume $(\mathrm{HB})$ and $(\mathrm{H} k)$ are valid. Let $\left(\pi, V_{\pi}\right) \in \mathcal{E}$. Suppose $\left(\pi, V_{\pi}\right)$ contains $\left(K_{\Sigma}^{+}, \chi_{\Gamma}\right)$. Then $\left(\pi, V_{\pi}\right)$ also contains $\left(K_{\vdash}, \chi_{\Gamma}\right)$ if

(1) $U_{y, 0}^{0}=U_{y, 0^{+}}^{0}$ or

(2) there is a nonzero vector in the $\left(K_{\Sigma}^{+}, \chi_{\Gamma}\right)$-isotypic component of $V_{\pi}$ which is invariant under $U_{y, 0}^{0}$.

The following lemma is a preparation for Proposition 13.14 and Proposition 14.5. For any open compact subgroup $J \subset G$, we write $J_{\ell}:=J \cap \bar{U}, J_{M}:=J \cap M$ and $J_{u}:=J \cap U$.

\section{Lemma 13.13.}

(1) $\tilde{K}_{\vdash}^{d}=\tilde{K}_{\vdash \ell}^{d} \tilde{K}_{\vdash M}^{d} \tilde{K}_{\vdash u}^{d}=\tilde{K}_{\vdash M}^{d} K_{\vdash \ell}^{d} K_{\vdash u}^{d}=K_{\vdash \ell}^{d} K_{\vdash u}^{d} \tilde{K}_{\vdash M}^{d} \cdot$

(2) $\tilde{K}_{\vdash}=\tilde{K}_{\vdash \ell} \tilde{K}_{\vdash M} \tilde{K}_{\vdash u}$.

(3) Suppose $\mathbf{G}^{0} \subset \mathbf{M}$. Then, $K_{\vdash}=K_{\vdash \ell} K_{\vdash M} K_{\vdash u}$.

Proof. Proving (3), we first note that each $f^{i}, i=1, \cdots, d$, is concave and positive. From [9, (6.4.9)] and the concavity of $f^{i}$, we deduce that $\mathbf{G}^{i}(E)_{y, f^{i}}$ is decomposable with respect to $\mathbf{P}(E)=\mathbf{M}(E) \mathbf{U}(E)$. That is,

$$
\mathbf{G}^{i}(E)_{y, f^{i}}=\left(\mathbf{G}^{i}(E)_{y, f^{i}} \cap \overline{\mathbf{U}}(E)\right) \cdot\left(\mathbf{G}^{i}(E)_{y, f^{i}} \cap \mathbf{M}(E)\right) \cdot\left(\mathbf{G}^{i}(E)_{y, f^{i}} \cap \mathbf{U}(E)\right) .
$$

From the positivity of $f^{i}$, we have $K_{\vdash}^{i}=G_{y, f}^{i}=\mathbf{G}^{i}(E)_{y, f^{i}}^{\operatorname{Gal}(E / k)}$. Since $\mathbf{M}$, $\mathbf{U}$, and $\overline{\mathbf{U}}$ are $\operatorname{Gal}(E / k)$-stable, $K_{\vdash}^{i}=G_{y, f}^{i}$ is also decomposable with respect to $P=M U$, that is, $K_{\vdash}^{i}=K_{\vdash \ell}^{i} K_{\vdash M}^{i} K_{\vdash u}^{i}$. If $i=0$, since $\mathbf{G}^{0} \subset \mathbf{M}, K_{\vdash}^{0}=K_{\vdash M}^{0}$. 
Now, let $K_{\vdash}(i)=K_{\vdash}^{i} \cdots K_{\vdash}^{d}$. We claim that each $K_{\vdash}(i)$ is decomposable with respect to $P=M U$. If $i=d$, it follows from the previous paragraph. Suppose $K_{\vdash}(i+1)$ is decomposable. Note that $K_{\vdash}(i+1)$ is normalized by $K_{\vdash}^{i}$ and $K_{\vdash}(i)=K_{\vdash}^{i} K_{\vdash}(i+1)$. Let $g \in K_{\vdash}(i)$. Then $g=a b$ for some $a \in K_{\vdash}^{i}$ and $b \in K_{\vdash}(i+1)$. Write $a=a_{\ell} a_{M} a_{u}$ compatible with the decomposition of $K_{\vdash}^{i}$. Since $b^{\prime}:=a_{u} b a_{u}^{-1} \in K_{\vdash}(i+1)$, we can also write $b^{\prime}=b_{\ell}^{\prime} b_{M}^{\prime} b_{u}^{\prime}$. Then, $a b=$ $a_{\ell} a_{M} a_{u} b a_{u}^{-1} a_{u}=\left(a_{\ell} a_{M} b_{\ell}^{\prime} a_{M}^{-1}\right)\left(a_{M} b_{M}^{\prime}\right)\left(b_{u}^{\prime} a_{u}\right)$, where $a_{\ell} a_{M} b_{\ell}^{\prime} a_{M}^{-1} \in K_{\vdash}(i)_{\ell}, a_{M} b_{M}^{\prime} \in$ $K_{\vdash}(i)_{M}$ and $b_{u}^{\prime} a_{u} \in K_{\vdash}(i)_{u}$. Hence, $K_{\vdash}(i)$ is decomposable. Since $K_{\vdash}=K_{\vdash}(0)$, $K_{\vdash}$ is also decomposable.

The second and the third equalities in (1) follow from $\tilde{K}_{\vdash \ell}^{d}=K_{\vdash \ell}^{d}$ and $\tilde{K}_{\vdash u}^{d}=$ $K_{\vdash u}^{d}$. The other equalities are proved similarly as in (3).

Proposition 13.14. Let $\Sigma=\Sigma_{y}$ be a strongly good $G$-datum of positive depth. Suppose $(\mathrm{HB})$ is valid. The support of $\mathcal{H}\left(G / / K_{\vdash}, \chi_{\Gamma}\right)$ is contained in $\tilde{K}_{\vdash} G^{0} \tilde{K}_{\vdash}$.

The following is a modification of the proof of [38, (4.1)].

Proof. We prove the proposition by induction. If $d=0$, it is obvious. Now, assume $d \geq 1$. Let $\Sigma^{\prime}=\left(\overrightarrow{\mathbf{G}}^{\prime}, y, \vec{r}^{\prime}, \vec{\phi}^{\prime}\right)$ be such that $\overrightarrow{\mathbf{G}}^{\prime}=\left(\mathbf{G}^{0}, \cdots, \mathbf{G}^{d-1}\right), \vec{\phi}^{\prime}=$ $\left(\phi_{0}, \cdots, \phi_{d-1}\right)$, and $\vec{r}^{\prime}=\left(r_{0}, \cdots, r_{d-1}\right)$. Then note that $\ell\left(\Sigma^{\prime}\right)=d-1$. Let

$$
K_{\vdash}^{\prime}:=K_{\vdash}^{0} K_{\vdash}^{1} \cdots K_{\vdash}^{d-1} \quad \text { and } \quad \tilde{K}_{\vdash}^{\prime}:=\tilde{K}_{\vdash}^{0} \tilde{K}_{\vdash}^{1} \cdots \tilde{K}_{\vdash}^{d-1} .
$$

Suppose $g \in G$ is in the support of $\mathcal{H}\left(G / / K_{\vdash}, \chi_{\Gamma}\right)$. Since $\phi_{d} \mid K_{\vdash}$ is the restriction of $\phi_{d}$ which is defined on the whole of $G, g$ also intertwines $\theta^{\prime}=\left(\chi_{\Gamma} \phi_{d}^{-1}\right) \mid K_{\vdash}=$ $\prod_{j=0}^{d-1} \chi_{\Gamma_{j}} \mid K_{\vdash}$. Therefore, $g$ also intertwines $\theta^{\prime} \mid K_{\vdash}^{d}$. Note that if $r_{j} \leq s, \chi_{\Gamma_{j}}$ is trivial on $K_{\vdash}^{d}$ and $\theta^{\prime} \mid K_{\vdash}^{d}=\chi_{\Gamma_{d-1}+Y}$ for $Y=\sum_{r_{j}>s_{d-1}} \Gamma_{j}$. By Lemma 13.5 and Lemma 13.13, there are $j_{1}, j_{2} \in \tilde{K}_{\vdash M}^{d}=M_{y, s_{d-1}}^{d}, h_{1}, h_{2} \in K_{\vdash \ell}^{d} K_{\vdash u}^{d}$ and $g^{\prime} \in$ $G^{d-1}$ such that $g=h_{1} j_{1} g^{\prime} j_{2} h_{2}$. Since $h_{1}, h_{2} \in K_{\vdash}, j_{1} g^{\prime} j_{2}$ intertwines $\theta^{\prime}$. Now, from Lemma 13.15(1), we may further assume $j_{1}, j_{2} \in J_{M}^{d}$, where $J_{M}^{d}$ is as in that lemma. Then, by Lemma 13.15(2), $g^{\prime}$ also intertwines $\theta^{\prime} \mid K_{\vdash}^{\prime}=\chi_{\Gamma^{\prime}}$ where $\Gamma^{\prime}=\Gamma_{d-1}+\Gamma_{d-2}+\cdots+\Gamma_{0}$. By the induction hypothesis, $g^{\prime} \in \tilde{K}_{\vdash}^{\prime} G^{0} \tilde{K}_{\vdash}^{\prime}$. Hence $g \in \tilde{K}_{\vdash}^{d} \tilde{K}_{\vdash}^{\prime} G^{0} \tilde{K}_{\vdash}^{\prime} \tilde{K}_{\vdash}^{d}=\tilde{K}_{\vdash} G^{0} \tilde{K}_{\vdash}$.

Lemma 13.15. We keep the notation from the proof of Proposition 13.14, Let

$$
J_{M}^{d}=\left(\mathbf{M}^{d-1}, \mathbf{M}^{d}\right)(k)_{y,\left(r_{d-1}, s_{d-1}\right)} .
$$

(1) $M_{y, s_{d-1}}^{d} G^{d-1} M_{y, s_{d-1}}^{d} \subset J_{M}^{d} G^{d-1} J_{M}^{d}$.

(2) If $g \in G^{d-1}$ intertwines $\theta^{\prime}, j \in J_{M}^{d}$, then $g j$ and $j g$ also intertwines $\theta^{\prime}$.

Proof. (1) follows from

$$
M_{y, s_{d-1}}^{d}=M_{x, s_{d-1}}^{d-1} J_{M}^{d}=J_{M}^{d} M_{x, s_{d-1}}^{d-1} \subset J_{M}^{d} G^{d-1} \subset J_{M}^{d} G^{d-1} J_{M}^{d} .
$$

(2) can be proved as in [38, (4.3)].

\section{Supercuspidality I}

Let $\Sigma=\Sigma_{y}$ be a strongly good $G$-datum of positive depth, and let $\mathbf{M}, \mathbf{P}=\mathbf{M U}$ and $K_{\vdash}$ be as in $\$ 13.9$. In this section, we prove that if there is a supercuspidal representation $\left(\pi, V_{\pi}\right)$ such that $\left(K_{\Sigma}^{+}, \chi_{\Gamma}\right)<\left(\pi, V_{\pi}\right), \mathbf{Z}^{0} / \mathbf{Z}^{d}$ is $k$-anisotropic (see Proposition 14.5). 
We first recall the following from [8]. For any open compact subgroup $J \subset G$, $J_{\ell}, J_{M}$ and $J_{u}$ are defined as before (see Lemma 13.13).

Definition $14.1([8,(6.16)])$. Let $P=M U$ be as above. Suppose $J$ is an open compact subgroup decomposable with respect to $P=M U$, that is, $J=J_{\ell} J_{M} J_{u}$. Then $\zeta \in Z_{M}$ is strongly positive relative to $(P, J)$ if

(1) $\zeta J_{u} \zeta^{-1} \subset J_{u}, \quad \zeta^{-1} J_{\ell} \zeta \subset J_{\ell}$;

(2) for any compact open subgroups $H_{1}, H_{2}$ of $U$, there exists an integer $m \geq 0$ such that $\zeta^{m} H_{1} \zeta^{-m} \subset H_{2}$;

(3) for any compact open subgroups $K_{1}, K_{2}$ of $\bar{U}$, there exists an integer $m \geq 0$ such that $\zeta^{-m} K_{1} \zeta^{m} \subset K_{2}$.

Combining [8, (6.14)] and the remark after [8, (6.16)], a strongly positive element always exists relative to $(P, J)$ such that $J=J_{\ell} J_{M} J_{u}$.

The following can be regarded as a corollary of the proof of [8, (6.10)].

Lemma 14.2. Let $J, \bar{U}$ and $P=M U$ be as in Definition 14.1, Then, $J M J \cap \bar{U}=$ $J_{\ell}$.

Proof. $\supset$ is obvious. To prove $\subset$, suppose $u \in J M J \cap \bar{U}$. Let $t \in M$ be so that $u \in J t J$. Then, there are $j, h \in J$ so that $u j=h t$. Write $j=j_{\ell} j_{M} j_{u}$ and $h=h_{\ell} h_{M} h_{u}$ compatible with the decomposition $J=J_{\ell} J_{M} J_{u}$. Then, $\left(u j_{\ell}\right) j_{M} j_{u}=$ $h_{\ell}\left(h_{M} t\right)\left(t^{-1} h_{u} t\right)$. Hence, by the uniqueness of Iwahori decomposition, we have $u=h_{\ell} j_{\ell}^{-1} \in J_{\ell}$, and $\subset$ follows.

Proposition 14.3. Assume $(\mathrm{HB})$ is valid. Suppose $\mathbf{G}^{0} \subset \mathbf{M} \subsetneq \mathbf{G}$. Then there is a strongly positive element $\zeta$ relative to $\left(P, K_{\vdash}\right)$ such that an element $f_{\zeta} \in$ $\mathcal{H}\left(G / / K_{\vdash}, \chi_{\Gamma}\right)$ supported on $K_{\vdash} \zeta K_{\vdash}$ is invertible.

Proof. We fix a Haar measure on $G$ so that $\operatorname{vol}\left(K_{\vdash}\right)=1$. From $\mathbf{G}^{0} \subset \mathbf{M}$ and Proposition 13.14, the support of $\mathcal{H}:=\mathcal{H}\left(G / / K_{\vdash}, \chi_{\Gamma}\right)$ is contained in $K_{\vdash} M K_{\vdash}$. Let $\zeta \in Z_{M} \subset G^{0}$ be a strongly positive element such that $\bar{U}=\bigcup_{n \geq 0} \zeta^{n} K_{\vdash \ell} \zeta^{-n}$. For $j=j_{\ell} j_{M} j_{u} \in K_{\vdash} \cap\left(\zeta K_{\vdash} \zeta^{-1}\right)$, since

$$
{ }^{\zeta} \chi_{\Gamma}(j)=\chi_{\Gamma}\left(\zeta^{-1} j \zeta\right)=\chi_{\Gamma}\left(\zeta^{-1} j_{\ell} \zeta\right) \chi_{\Gamma}\left(\zeta^{-1} j_{M} \zeta\right) \chi_{\Gamma}\left(\zeta^{-1} j_{u} \zeta\right)=\chi_{\Gamma}\left(j_{M}\right)=\chi_{\Gamma}(j),
$$

we have $\zeta \in \operatorname{Supp}(\mathcal{H})$. Here, the third equality follows from $K_{\vdash \ell}, K_{\vdash u} \subset \operatorname{ker}\left(\chi_{\Gamma}\right)$ and $\zeta^{-1} j_{M} \zeta=j_{M}$. Similarly, $\zeta^{-1} \in \operatorname{Supp}(\mathcal{H})$. Let $f_{\zeta}\left(\right.$ resp. $\left.f_{\zeta^{-1}}\right)$ be an element of $\mathcal{H}$ which is the function supported on $K_{\vdash} \zeta K_{\vdash}\left(\right.$ resp. $\left.K_{\vdash} \zeta^{-1} K_{\vdash}\right)$ with $f_{\zeta}(\zeta)=1$ (resp. $f_{\zeta^{-1}}\left(\zeta^{-1}\right)=1$ ). We claim

$$
f_{\zeta} * f_{\zeta^{-1}}=f_{\zeta^{-1}} * f_{\zeta}=\operatorname{vol}\left(K_{\vdash} \zeta K_{\vdash}\right) f_{1}
$$

where $f_{1}$ is the identity in $\mathcal{H}$ given by the function supported on $K_{\vdash}$ with $f_{1}(1)=1$. Note that $\operatorname{Supp}\left(f_{\zeta} * f_{\zeta^{-1}}\right) \subset\left(K_{\vdash} \zeta K_{\vdash} \zeta^{-1} K_{\vdash}\right) \cap\left(\tilde{K}_{\vdash} G^{0} \tilde{K}_{\vdash}\right)$. From Lemma 14.4. $\operatorname{Supp}\left(f_{\zeta^{*}} * f_{\zeta^{-1}}\right) \subset K_{\vdash}$. Hence $f_{\zeta^{*}} * f_{\zeta^{-1}}=c \cdot f_{1}$ for some constant $c$. Evaluating both sides at the identity, $c=\operatorname{vol}\left(K_{\vdash} \zeta K_{\vdash}\right)$. Similar computation shows that $f_{\zeta^{-1}} * f_{\zeta}=$ $\operatorname{vol}\left(K_{\vdash} \zeta K_{\vdash}\right) f_{1}$. Hence, the inverse of $f_{\zeta}$ is $\frac{1}{\operatorname{vol}\left(K_{\vdash} \zeta K_{\vdash}\right)} f_{\zeta^{-1}}$.

Lemma 14.4. We keep the notation and the situation from the proof of Proposition 14.3 .

$$
\left(K_{\vdash} \zeta K_{\vdash} \zeta^{-1} K_{\vdash}\right) \cap\left(\tilde{K}_{\vdash} G^{0} \tilde{K}_{\vdash}\right)=K_{\vdash}=\left(K_{\vdash} \zeta^{-1} K_{\vdash} \zeta K_{\vdash}\right) \cap\left(\tilde{K}_{\vdash} G^{0} \tilde{K}_{\vdash}\right) .
$$


Proof. It is obvious that $K_{\vdash} \subset\left(K_{\vdash} \zeta K_{\vdash} \zeta^{-1} K_{\vdash}\right) \cap\left(\tilde{K}_{\vdash} G^{0} \tilde{K}_{\vdash}\right)$. Suppose $g \in$ $\left(K_{\vdash} \zeta K_{\vdash} \zeta^{-1} K_{\vdash}\right) \cap\left(\tilde{K}_{\vdash} G^{0} \tilde{K}_{\vdash}\right)$. Since $\zeta K_{\vdash M} \zeta^{-1}=K_{\vdash M}$ and $\zeta K_{\vdash u} \zeta^{-1} \subset K_{\vdash u}$, we can write $g=j_{1} \zeta j_{\ell} \zeta^{-1} j_{2}$ for some $j_{1}, j_{2} \in K_{\vdash}$ and $j_{\ell} \in K_{\vdash \ell}$. Since $\zeta j_{\ell} \zeta^{-1} \in$ $\tilde{K}_{\vdash} G^{0} \tilde{K}_{\vdash} \cap \bar{U}$, by Lemma 14.2, $\zeta j_{\ell} \zeta^{-1} \in \tilde{K}_{\vdash \ell}=K_{\vdash \ell}$. Hence, $g \in K_{\vdash}$. The other equality can be proved in a similar way.

Proposition 14.5. Assume $(\mathrm{HB})$ and $(\mathrm{H} k)$ are valid. Let $\left(\pi, V_{\pi}\right)$ be a supercuspidal representation. Let $\Sigma=(\overrightarrow{\mathbf{G}}, y, \vec{r}, \vec{\phi})$ be a strongly good $G$-datum of positive depth. Suppose that $\left(K_{\Sigma}^{+}, \phi_{\Sigma}\right)$ is contained in $\left(\pi, V_{\pi}\right)$. Then, $\mathbf{Z}^{0} / \mathbf{Z}^{d}$ is $k$-anisotropic.

Proof. Suppose $\mathbf{Z}^{0} / \mathbf{Z}^{d}$ is not anisotropic. Then, there is a $k$-split subtorus $\mathbf{S}$ of $\mathbf{Z}^{0}$ such that $\mathbf{M}=\mathbf{C}_{\mathbf{G}}(\mathbf{S})$ is a proper $k$-Levi subgroup of $\mathbf{G}$. Note that $\mathbf{G}^{0} \subset \mathbf{M}$. Hence $y \in \mathcal{B}(\mathbf{M}, k)$. Fix a $k$-parabolic subgroup $\mathbf{P}$ with Levi decomposition $\mathbf{P}=\mathbf{M U}$, and let $\overline{\mathbf{U}}$ be the unipotent subgroup opposite to $\mathbf{U}$. Since $\mathbf{G}^{0} \subset \mathbf{M}, U_{y, 0}^{0}=1$ and $\left(\pi, V_{\pi}\right)$ contains $\left(K_{\vdash}, \chi_{\Gamma}\right)$ by Corollary 13.12 , Now, consider $\mathcal{H}:=\mathcal{H}\left(G / / K_{\vdash}, \chi_{\Gamma}\right)$. By Proposition 14.3, there is a strongly positive element $\zeta$ relative to $\left(P, K_{\vdash}\right)$ such that $f_{\zeta}$, an element of $\mathcal{H}\left(G / / K_{\vdash}, \chi_{\Gamma}\right)$ supported on $K_{\vdash} \zeta K_{\vdash}$, is invertible. This implies that the Jacquet map restricted to $V_{\pi}^{\chi_{\Gamma}}$, the $\left(K_{\vdash}, \chi_{\Gamma}\right)$-isotypic component in $V_{\pi}$, is injectively mapped into $V_{\pi U}^{\chi_{\Gamma}}$, the $\left(K_{\vdash} \cap M, \chi_{\Gamma}\right)$-isotypic component in the Jacquet module $V_{\pi U}\left([\underline{8})\right.$. Since $V_{\pi}^{\chi_{\Gamma}} \neq 0$, this implies that the Jacquet module $V_{\pi U}$ of $V_{\pi}$ with respect to $U$ is nontrivial, which contradicts the supercuspidality of $\left(\pi, V_{\pi}\right)$. Hence, $\mathbf{Z}^{0} / \mathbf{Z}^{d}$ is $k$-anisotropic.

\section{DePth ZERO TYPES}

In this section and the following one, we deduce some facts that we need for 17 (Proposition 17.2). Most results here are from 31.

15.1. Let $\left(\mathbf{G}^{\prime}, \mathbf{G}\right)$ be a tamely ramified twisted Levi sequence, which splits over a tamely ramfied extension $E$. For any $y \in \mathcal{B}\left(\mathbf{G}^{\prime}, k\right)$, let $\mathrm{M}_{y}^{\prime}$ be the quotient of the reduction mod $\mathfrak{p}_{k}$ of the $\mathcal{O}_{k}$-group scheme $\mathrm{P}_{y}^{\prime}$ associated to $G_{y, 0}^{\prime}$ by its unipotent radical. Then, $G_{y, 0}^{\prime} / G_{y, 0^{+}}^{\prime} \simeq \mathrm{M}_{y}^{\prime}\left(\mathbb{F}_{q}\right)$.

Let $\mathbf{T} \subset \mathbf{G}^{\prime}$ be an $E$-split maximal $k$-torus such that $y \in \mathcal{A}\left(\mathbf{G}^{\prime}, \mathbf{T}, k\right)$ and $\mathcal{A}\left(\mathbf{G}^{\prime}, \mathbf{T}, k\right)$ is of maximal dimension. Let $\mathbf{S}$ be a maximal $k$-split subtorus in $\mathbf{T}$. We attach a $k$-Levi subgroup $\mathbf{M}$ of $\mathbf{G}$ to $G_{y, 0}^{\prime}$ (see also [31, (6.3)]): first note that $\mathbf{S}$ gives rise to a maximal $\mathbb{F}_{q}$-torus $\mathrm{S}$ of $\mathrm{M}_{y}^{\prime}$. Let $\mathrm{C}$ be the maximal $\mathbb{F}_{q^{-}}$-split torus contained in the center of $\mathbf{M}_{y}^{\prime}$. Lift $\mathbf{C}$ to $\mathbf{S}$ to get a subtorus $\mathbf{C}$ of $\mathbf{S}$. Let $\mathbf{M}$ be the centralizer of $\mathbf{C}$ in $\mathbf{G}$. Note that since $\mathbf{T} \subset \mathbf{M}, y \in \mathcal{B}(\mathbf{M}, k)$. Moreover, if $G_{y, 0}^{\prime}$ is not a maximal parahoric subgroup of $G^{\prime}, M$ is a proper $k$-Levi subgroup.

For a given $G_{y, 0}^{\prime}$, if $\mathbf{M}$ is chosen in the above manner, we will say that $\mathbf{M}$ is adapted for $G_{y, 0}^{\prime}$. In [31, (6.3)], the case $\mathbf{G}^{\prime}=\mathbf{G}$ is considered.

Remark 15.2. We keep the situation from $\$ 15.1$. Let $\mathbf{M}^{\prime}:=\mathbf{M} \cap \mathbf{G}^{\prime}=\mathbf{C}_{\mathbf{G}^{\prime}}(\mathbf{C})$. Then, $\mathbf{M}^{\prime}$ is a $k$-Levi subgroup of $\mathbf{G}^{\prime}$ associated to $G_{y, 0}^{\prime}$ as in [31, (6.3)]. Set $M_{y, r}^{\prime}:=M \cap G_{y, r}^{\prime}$ for $r \in \tilde{\mathbb{R}}$ as before. Then, by [31, (6.4)], we have the following:

(1) $M_{y, 0}^{\prime}$ is a maximal parahoric subgroup of $M^{\prime}$.

(2) $G_{y, 0}^{\prime} / G_{y, 0^{+}}^{\prime} \simeq M_{y, 0}^{\prime} / M_{y, 0^{+}}^{\prime} \simeq \mathrm{M}_{y}^{\prime}\left(\mathbb{F}_{q}\right)$.

15.3. Let $\left(\pi, V_{\pi}\right)$ be a smooth representation of $G$. Let $x, y, y^{\prime} \in \mathcal{B}(\mathbf{G}, k)$ be such that $G_{y, 0}$ and $G_{y^{\prime}, 0}$ are proper parahoric subgroups in $G_{x, 0}$. Let $\mathbf{M}$ be the Levi subgroup adapted for $G_{y, 0}$ with $x, y, y^{\prime} \in \mathcal{B}(\mathbf{M}, k)$. Suppose $M_{y, 0}=M_{y^{\prime}, 0}$, and 
suppose $G_{y, 0}$ and $G_{y^{\prime}, 0}$ project onto opposite parabolic subgroups in $G_{x, 0} / G_{x, 0^{+}}$ with common Levi factor $\left(G_{y, 0} \cap G_{y^{\prime}, 0}\right) / G_{x, 0^{+}} \simeq M_{y, 0} / M_{y, 0^{+}}=M_{y^{\prime}, 0} / M_{y^{\prime}, 0^{+}}$. Suppose $P=M U$ and $\bar{P}=M \bar{U}$ are two opposite parabolic subgroups in $G$ so that $G_{y, 0} \cap P=M_{y, 0} U_{y, 0}$ projects onto $G_{y, 0} / G_{x, 0^{+}}$and $G_{y^{\prime}, 0} \cap \bar{P}=M_{y^{\prime}, 0} \bar{U}_{y^{\prime}, 0}$ projects onto $G_{y^{\prime}, 0} / G_{x, 0^{+}}$.

The following is a corollary of the proof of [31, (6.7)], which in turn results from [31, (6.1)]. Note that $G_{y, 0} \cap U=G_{y, 0^{+}} \cap U$.

Lemma 15.4. We keep the notation and situation from \$15.3. If a nonzero vector $v \in V_{\pi}$ is fixed under $G_{y^{\prime}, 0^{+}}$, then the following integral does not vanish:

$$
\int_{U \cap G_{y, 0}} \pi(n) v d n \neq 0 \text {. }
$$

Let $\chi$ be a quasi character of $G$ and let $\pi_{\chi}:=\chi \otimes \pi$. Suppose $\chi$ is trivial on $U$ and $\bar{U}$. Then, if $v$ is a nonzero vector in $V_{\pi_{\chi}}^{\left(G_{y^{\prime}, 0^{+}}, \chi\right)}$,

$$
\int_{U \cap G_{y, 0}} \pi_{\chi}(n) v d n=\int_{U \cap G_{y, 0}} \pi(n) v d n \neq 0 .
$$

\section{HeisenBERG REPRESENTATION}

16.1. The case $\left(\mathbf{G}^{\prime}, \mathbf{G}\right)$. Let $\left(\mathbf{G}^{\prime}, \mathbf{G}\right)$ be a tamely ramified twisted Levi sequence, and let $y \in \mathcal{B}\left(\mathbf{G}^{\prime}, k\right)$. Let $\mathbf{T}, \mathbf{M}, \mathbf{U}, \overline{\mathbf{U}}$ and $\phi$ be as in 13.2 , and let $\mathbf{M}^{\prime}=\mathbf{G} \cap \mathbf{M}$. Define concave functions $h_{+}, h: \Phi(\mathbf{G}, \mathbf{T}, E) \cup\{0\} \longrightarrow \tilde{\mathbb{R}}$ as

$$
h_{+}(a)= \begin{cases}r & \text { if } a \in \Phi(\mathbf{M}, \mathbf{T}, E) \cup\{0\}, \\ s & \text { if } a \in \Phi\left(\mathbf{G}^{\prime}, \mathbf{T}, E\right) \backslash \Phi(\mathbf{M}, \mathbf{T}, E), \\ s^{+} & \text {otherwise }\end{cases}
$$

and

$$
h(a)= \begin{cases}r & \text { if } a \in \Phi(\mathbf{M}, \mathbf{T}, E) \cup\{0\}, \\ s & \text { if } a \in \Phi\left(\mathbf{G}^{\prime}, \mathbf{T}, E\right) \backslash \Phi(\mathbf{M}, \mathbf{T}, E), \\ s & \text { otherwise. }\end{cases}
$$

Let $\tilde{J}_{+}:=G_{y, h_{+}}$and $\tilde{J}:=G_{y, h}$. Note that $\tilde{J} \cap U=G_{y, s} \cap U$. Then since $\phi$ defines a character $\hat{\phi}$ on $G_{y, 0^{+}}^{\prime} G_{y, s^{+}}($see $\$ 5.8), \hat{\phi}$ can be restricted to $\tilde{J}_{+} \subset G_{y, 0^{+}}^{\prime} G_{y, s^{+}}$. Let $N:=\operatorname{ker}(\hat{\phi})$.

\section{Lemma 16.2.}

(1) The pairing $\langle a, b\rangle=\hat{\phi}\left(a b a^{-1} b^{-1}\right)$ defined on $\tilde{J} / \tilde{J}_{+} \times \tilde{J} / \tilde{J}_{+}$is nondegenerate.

(2) $\tilde{J} / N$ is a Heisenberg p-group with center $\tilde{J}_{+} / N$.

The proof is similar to that of [38, (11.1)]. We will not repeat it here.

Lemma 16.3. Let $\left(\phi^{\mathrm{h}}, V_{\phi^{\mathrm{h}}}\right)$ be a representation of the Heisenberg group $\tilde{J} / N$. We use the same notation $\phi^{\mathrm{h}}$ for the inflated representation of $\tilde{J}$. Let $v \in V_{\phi^{\mathrm{h}}}$. If $v \neq 0$ and it is fixed by $\tilde{J} \cap \bar{U}$, then the following integral does not vanish:

$$
\int_{\tilde{J} \cap U} \phi^{\mathrm{h}}(u) v d u \neq 0 .
$$

Proof. Note that $(\tilde{J} \cap \bar{U}) /\left(\tilde{J}_{+} \cap \bar{U}\right)$ and $(\tilde{J} \cap U) /\left(\tilde{J}_{+} \cap U\right)$ are isotropic subspaces of $\tilde{J} / \tilde{J}_{+}$such that their sum form a complete polarization of $\tilde{J} / \tilde{J}_{+}$. Then the above lemma is a result of the following general fact on Heisenberg representations. 
Lemma 16.4. Let $\mathbb{H}=\mathbb{W} \oplus \mathbb{F}_{q}$ be a Heisenberg group where $\mathbb{W}$ is a symplectic space over $\mathbb{F}_{q}$. Let $\mathbb{W}=W_{1} \oplus W_{2}$ be a complete polarization of $\mathbb{W}$. Let $\left(\rho, V_{\rho}\right)$ be a finite-dimensional representation of $\mathbb{H}$. If a nonzero vector $v \in V_{\rho}$ is fixed under $W_{1}$, then

$$
\int_{W_{2}} \rho\left(w_{2}\right) v d w_{2} \neq 0
$$

Proof. Without loss of generality, we may assume that $\rho$ is irreducible. If $\rho$ is the trivial representation, the lemma is trivial. Otherwise, there is a nontrivial character $\chi$ of the center $\mathbb{F}_{q}$ such that $\rho$ is isomorphic to the Heisenberg representation $\chi^{\mathrm{h}}$ of $\mathbb{H}$ with the central character $\chi$. Then, we can realize $\chi^{\mathrm{h}}$ on the function space $C\left(W_{1}\right)$ as follows: for $f \in C\left(W_{1}\right)$,

$$
\begin{array}{ll}
\chi^{\mathrm{h}}\left(w_{1}\right) f(w)=f\left(w+w_{1}\right), & \text { for } w_{1} \in W_{1}, \\
\chi^{\mathrm{h}}\left(w_{2}\right) f(w)=\chi\left(\frac{1}{2}\left\langle w, w_{2}\right\rangle\right) f(w), & \text { for } w_{2} \in W_{2}, \\
\chi^{\mathrm{h}}(z) f(w)=\chi(z) f(w), & \text { for } z \in \mathbb{F}_{q} .
\end{array}
$$

Denote the characteristic function supported at $w \in W_{1}$ by $f_{w}$. Suppose $f \in C\left(W_{1}\right)$ is a nonzero function invariant by $W_{1}$. Then from the above formula, $f$ is a nonzero constant function, that is, $f=c \sum_{w \in W_{1}} f_{w}$ for some constant $c \neq 0$. Now, we have

$$
\begin{aligned}
\int_{W_{2}} \chi^{\mathrm{h}}\left(w_{2}\right) f d w_{2} & =\int_{W_{2}} \chi^{\mathrm{h}}\left(w_{2}\right)\left(\sum_{w} c f_{w}\right) d w_{2} \\
& =\int_{W_{2}} \sum_{w} c\left(\chi\left(\frac{1}{2}\left\langle w, w_{2}\right\rangle\right) f_{w}\right) d w_{2} \\
& =\int_{W_{2}} c f_{0} d w_{2}=\operatorname{vol}\left(W_{2}\right) \cdot c \cdot f_{0} \neq 0 .
\end{aligned}
$$

Corollary 16.5. We keep the notation from Lemma 16.3, Let $\chi$ be a character of $\tilde{J}$ such that $\chi$ is trivial on $\tilde{J} \cap U$ and $\tilde{J} \cap \bar{U}$. Let $v \in V_{\chi \otimes \phi^{\mathrm{h}}}$. If $v \neq 0$ and if it is fixed by $\tilde{J} \cap \bar{U}$, then

$$
\int_{\tilde{J} \cap U}\left(\chi \otimes \phi^{\mathrm{h}}\right)(u) v d u \neq 0 .
$$

16.6. The general case. Let $\Sigma_{y}:=(\overrightarrow{\mathbf{G}}, y, \vec{r}, \vec{\phi})$ be a good $G$-datum of positive depth. Let $\mathbf{T}, \mathbf{M}, \mathbf{U}$ and $\overline{\mathbf{U}}$ be as in $\$ 13.9$. Recall $\mathbf{M}^{i}=\mathbf{M} \cap \mathbf{G}^{i}, \mathbf{U}^{i}=\mathbf{U} \cap \mathbf{G}^{i}$ and $\overline{\mathbf{U}}^{i}=\overline{\mathbf{U}} \cap \mathbf{G}^{i}$. For $i=1, \cdots, d$, define concave functions $h_{+}^{i}, h^{i}: \Phi\left(\mathbf{G}^{i}, \mathbf{T}, E\right) \cup$ $\{0\} \longrightarrow \tilde{\mathbb{R}}$ as

$$
h_{+}^{i}(a)= \begin{cases}r_{i-1} & \text { if } a \in \Phi\left(\mathbf{M}^{i}, \mathbf{T}, E\right) \cup\{0\}, \\ s_{i-1} & \text { if } a \in \Phi\left(\mathbf{G}^{i-1}, \mathbf{T}, E\right) \backslash \Phi\left(\mathbf{M}^{i}, \mathbf{T}, E\right), \\ s_{i-1}^{+} & \text {otherwise }\end{cases}
$$

and

$$
h^{i}(a)= \begin{cases}r_{i-1} & \text { if } a \in \Phi\left(\mathbf{M}^{i}, \mathbf{T}, E\right) \cup\{0\}, \\ s_{i-1} & \text { if } a \in \Phi\left(\mathbf{G}^{i-1}, \mathbf{T}, E\right) \backslash \Phi\left(\mathbf{M}^{i}, \mathbf{T}, E\right), \\ s_{i-1} & \text { otherwise. }\end{cases}
$$

We also define corresponding open compact subgroups of $G^{i}$ as follows: for $i=$ $1, \cdots, d$,

$$
\tilde{J}_{y+}^{i}=: G_{y, h_{+}^{i}}^{i} \subset G^{i}, \quad \tilde{J}_{y}^{i}=: G_{y, h^{i}}^{i} \subset G^{i} .
$$

Note that $\tilde{J}^{i} \cap U=G_{y, s_{i-1}}^{i} \cap U$. Then, $\phi_{i-1}$ defines a character $\hat{\phi}_{i-1}$ of $\tilde{J}_{y+}^{i}$ as in $\$ 5.8$ and each $\tilde{J}_{y}^{i} / \operatorname{ker}\left(\hat{\phi}_{i-1}\right)$ is a Heisenberg $p$-group with center $\tilde{J}_{y+}^{i} / \operatorname{ker}\left(\hat{\phi}_{i-1}\right)$. When $\Sigma$ is strongly good, $\hat{\phi}_{i-1}$ coincides with $\chi_{\Gamma_{i-1}}$. 


\section{Supercuspidality II}

In this section, let $\left(\pi, V_{\pi}\right)$ be a fixed supercuspidal representation of positive depth. Suppose $\Sigma=(\overrightarrow{\mathbf{G}}, y, \vec{r}, \vec{\phi})$ is a strongly good $G$-datum of positive depth such that $\left(K_{\Sigma}^{+}, \phi_{\Sigma}=\chi_{\Gamma}\right)$ is contained in $\left(\pi, V_{\pi}\right)$. Then, by Proposition 14.5, $\mathbf{Z}^{0} / \mathbf{Z}^{d}$ is $k$-anisotropic.

17.1. Consider the $\left(K_{\Sigma}^{+}, \chi_{\Gamma}\right)$ isotypic component $V_{\pi}^{\chi_{\Gamma}}$ of $V_{\pi}$. It can be found as the image of the projection

$$
v \longrightarrow \frac{1}{\operatorname{vol}\left(K_{\Sigma}^{+}\right)} \int_{K_{\Sigma}^{+}} \chi_{\Gamma}\left(g^{-1}\right) \pi(g) v d g .
$$

Recall that $G_{[y]}^{0}$ denotes the stabilizer in $G^{0}$ of the image $[y]$ of $y$ in the reduced building of $\mathbf{G}^{0}$. Since $G_{[y]}^{0}$ stabilizes $\left(K_{\Sigma}^{+}, \chi_{\Gamma}\right), V_{\pi}^{\chi_{\Gamma}}$ is a finite-dimensional representation of $G_{[y]}^{0}$. Let $\bar{\rho}$ be an irreducible subrepresentation of $G_{[y]}^{0}$ in $V_{\pi}^{\chi_{\Gamma}}$. Denote the character of $G_{[y]}^{0}$ given by $\prod_{i}\left(\phi_{i} \mid G_{[y]}^{0}\right)$ again by $\phi_{\Sigma}$. Then, $\rho=\phi_{\Sigma}^{-1} \otimes \bar{\rho}$ is an irreducible representation of $G_{[y]}^{0}$ which factors through $G_{y, 0^{+}}^{0}$.

Let $\tau$ be an irreducible subrepresentation of $\rho \mid G_{y, 0}^{0}$. Then $\tau$ induces an irreducible representation of $G_{y, 0}^{0} / G_{y, 0^{+}}^{0}$, which we again denote by $\tau$.

Proposition 17.2. Suppose $(\mathrm{H} k)$ and $(\mathrm{HB})$ are valid. We keep the notation from above.

(1) $G_{y, 0}^{0}$ is a maximal parahoric subgroup of $G^{0}$.

(2) $\tau$ is a cuspidal representation of $G_{y, 0}^{0} / G_{y, 0^{+}}^{0}$.

17.3. Proof of Proposition [17.2(1). We generalize the proof of [31, (6.7)].

Let $\mathbf{M}$ be a $k$-Levi subgroup of $\mathbf{G}$ adapted for $G_{y, 0}^{0}$. Suppose $G_{y, 0}^{0}$ is not maximal. Then, $\mathbf{M}$ is a proper Levi subgroup of $\mathbf{G}$. Let $\mathbf{C}$ be the center of $\mathbf{M}$. Let $\mathbf{P}$ be a $k$-parabolic subgroup with $\mathbf{P}=\mathbf{M U}$ and let $\overline{\mathbf{U}}$ be the unipotent subgroup opposite to $\mathbf{U}$. Then, $U \cap G_{y, 0}^{0}=U \cap G_{y, 0^{+}}^{0}$ and $\bar{U} \cap G_{y, 0}^{0}=\bar{U} \cap G_{y, 0^{+}}^{0}$. By Corollary 13.12(2), $\left(\pi, V_{\pi}\right)$ contains $\left(K_{y \vdash}, \chi_{\Gamma}\right)$.

For any $x \in \mathcal{B}\left(\mathbf{G}^{0}, k\right) \cap \mathcal{B}(\mathbf{M}, k)$, let $\Sigma_{x}=(\overrightarrow{\mathbf{G}}, x, \vec{r}, \vec{\phi})$. As in $\$ 13.9$, for $\Sigma_{x}$ and MU, we can define $K_{x \vdash}$, and $\vec{\phi}$ defines a character $\chi_{\Gamma}$ of $K_{x \vdash}$ realized by $\Gamma$.

Let $\beta: \mathbf{G L}_{1} \rightarrow \mathbf{C}$ be a one-parameter subgroup of $\mathbf{C}$ such that $\langle\alpha, \beta\rangle>0$ for every root $\alpha$ of $\mathbf{C}$ in the Lie algebra $\mathfrak{u}$ of $U$. Consider the ray $y(t)=y+t \beta, t \geq 0$, contained in the apartment $\mathcal{A}(\mathbf{G}, \mathbf{T}, k)$, emanating from the point $y$ in the direction of $\beta$. Then, one can verify the following (see also [31, (6.7)]):

(1) $M \cap K_{y(t) \vdash}=M_{y(t), \vec{s}^{+}}=M_{y, \vec{s}^{+}} ; U \cap K_{y(t) \vdash}=U_{y(t), \vec{s}} ; \bar{U} \cap K_{y(t) \vdash}=\bar{U}_{y(t), \vec{s}^{+}}$.

(2) If $t^{\prime} \geq t$, then $U_{y\left(t^{\prime}\right), \vec{s}} \supset U_{y(t), \vec{s}}$ and $\bar{U}_{y\left(t^{\prime}\right), \vec{s}^{+}} \subset \bar{U}_{y(t), \vec{s}^{+}}$.

(3) $U_{y(t), s_{i-1}}^{i}=\tilde{J}_{y(t)}^{i} \cap U$ and $U_{y(t), \vec{s}}=U_{y(t), 0}^{0} U_{y(t), s_{0}}^{1} \cdots U_{y(t), s_{d-1}}^{d}$.

(4) Any compact subgroup of $U$ is contained in $U_{y(t), \vec{s}}$ for $t$ sufficiently large.

(5) There is a sequence $0=t_{0}<t_{1}<t_{2}<\cdots$ tending to $\infty$ so that $U_{y(t), \vec{s}}, U_{y(t), \vec{s}^{+}}, \bar{U}_{y(t), \vec{s}}$ and $\bar{U}_{y(t), \vec{s}^{+}}$are constant on the open intervals $t_{i-1}<t<t_{i}(i \geq 1)$. Then, in fact, we have that $U_{y(t), \vec{s}}, \bar{U}_{y(t), \vec{s}^{+}}$are constant for $t_{i-1} \leq t<t_{i}$, and $U_{y(t), \vec{s}^{+}}, \bar{U}_{y(t), \vec{s}}$ are constant for $t_{i-1}<t \leq t_{i}$.

Let $x_{i}=y+t_{i} \beta$. Fix $t_{i}^{\prime} \in \mathbb{R}$ such that $t_{i-1}<t_{i}^{\prime}<t_{i}$. Let $y_{0}=x_{0}$ and $y_{i}=y+t_{i}^{\prime} \beta$. We observe that $U_{x_{0}, \vec{s}}=U_{y_{0}, \vec{s}}=U_{y_{1}, \vec{s}}, \bar{U}_{x_{0}, \vec{s}}=\bar{U}_{y_{0}, \vec{s}} \supset \bar{U}_{y_{1}, \vec{s}}$, and $U_{x_{j}, \vec{s}}=U_{y_{j+1}, \vec{s}}$. 
(6) $U_{y_{i}, \vec{s}^{+}}=U_{y_{i}, \vec{s}}$ and $\bar{U}_{y_{i}, \vec{s}^{+}}=\bar{U}_{y_{i}, \vec{s}}$, when $i \geq 1$.

Proving the first equality, $\subset$ is obvious. We may assume that $\Gamma$ is $k$-split without loss of generality. Let $\psi \in \Psi(\mathbf{G}, \mathbf{T}, k)$ such that the gradient $\dot{\psi}$ of $\psi$ is in $\Phi\left(\mathbf{U}^{j}, \mathbf{T}, k\right), j=0, \cdots, d$. Let $U_{\psi}$ be the root subgroup associated to $\psi$. Suppose $U_{\psi} \subset U_{y_{i}, \vec{s}}$. Then, by the definition of $y_{i}$ in (5), $U_{\psi} \subset U_{y_{i}-\epsilon \beta, \vec{s}}$ for sufficiently small $\epsilon>0$. Hence, $\psi\left(y_{i}-\epsilon \beta\right)=\psi\left(y_{i}\right)-\epsilon\langle\dot{\psi}, \beta\rangle \geq s_{j-1}$. Since $\langle\dot{\psi}, \beta\rangle>0, \psi\left(y_{i}\right)>s_{j-1}$. Hence $U_{\psi} \subset U_{y_{i}, \vec{s}^{+}}$, and $\supset$ follows. The second equality is similarly proved.

(7) $\tilde{J}_{x_{i}}^{\ell} \cap U=\tilde{J}_{y_{i+1}}^{\ell} \cap U=U_{y_{i+1}, s_{\ell-1}}^{\ell}$ and $\tilde{J}_{x_{i}}^{\ell} \cap \bar{U}=\tilde{J}_{y_{i}}^{\ell} \cap \bar{U}=\bar{U}_{y_{i}, s_{\ell-1}}^{\ell}$; $\tilde{J}_{x_{i}+}^{\ell} \cap U=U_{x_{i}, s_{\ell-1}^{+}}^{\ell} U_{x_{i}, s_{\ell-1}}^{\ell-1}$ and $\tilde{J}_{x_{i}+}^{\ell} \cap \bar{U}=\bar{U}_{x_{i}, s_{\ell-1}^{\ell}}^{\ell} \bar{U}_{x_{i}, s_{\ell-1}}^{\ell-1}, \quad \ell=1, \cdots, d$.

(8) $G_{y_{i}, 0}^{0}=\bar{U}_{y_{i}, 0}^{0} M_{y, 0}^{0} U_{y_{i}, 0}^{0}, \quad G_{y_{i}, 0^{+}}^{0}=\bar{U}_{y_{i}, 0}^{0} M_{y, 0^{+}}^{0} U_{y_{i}, 0}^{0}$ and $G_{x_{i}, 0^{+}}^{0}=$ $\bar{U}_{y_{i+1}, 0}^{0} M_{y, 0^{+}}^{0} U_{y_{i}, 0}^{0}$.

(9) $G_{x_{i}, 0}^{0}$ contains both $G_{y_{i}, 0}^{0}$ and $G_{y_{i+1}, 0}^{0}$. Suppose $G_{y_{i}, 0}^{0} \neq G_{y_{i+1}, 0}^{0}$. Then the images of $G_{y_{i}, 0}^{0}$ and $G_{y_{i+1}, 0}^{0}$ form the opposite parabolic subgroups in $G_{x_{i}, 0}^{0} / G_{x_{i}, 0^{+}}^{0}$ with the common Levi factor given by the image of $M_{y, 0}^{0}=$ $M_{y_{i}, 0}^{0}=M_{y_{i+1}, 0}^{0}$.

Let $v=v_{0} \in V_{\pi}^{\chi_{\Gamma}}$ be a nonzero element. Note that $U_{x_{j}, \vec{s}}=U_{y_{j+1}, \vec{s}}$ by (5). For $i=1,2, \cdots$, define

$$
v_{j}=\int_{U_{x_{j}, \vec{s}}} \pi(n) v_{j-1} d n=\int_{U_{y_{j+1}, \vec{s}}} \pi(n) v_{j-1} d n .
$$

Inductively, we will show

(i) $v_{j} \neq 0$

(ii) $v_{j}$ is a nonzero multiple of $\int_{U_{x_{j}, s}} \pi(n) v d n$;

(iii) $M_{y, \vec{s}^{+}}$acts on $v_{j}$ as a character represented by $\Gamma$;

(iv) for $j \geq 0$, we have $\operatorname{Stab}\left(v_{j}\right) \supset \bar{U}_{x_{j+1}, \vec{s}}=\bar{U}_{y_{j+1}, \vec{s}^{+}}$and $\operatorname{Stab}\left(v_{j}\right) \supset U_{x_{j+1}, \vec{s}^{+}}=$ $U_{y_{j+1}, \vec{s}^{+}}$;

(v) $G_{x_{j}, 0^{+}}^{0}$ acts on $v_{j}$ as a character represented by $\Gamma$;

(vi) $v_{j}=\int_{U_{x_{j}, 0}^{0}} \int_{U_{x_{j}, s_{0}}^{1}} \cdots \int_{U_{x_{j}, s_{d-1}}^{d}} \pi\left(u_{0} u_{1} \cdots u_{d}\right) v_{j-1} d u_{d} \cdots d u_{0}$.

The equality in (ii) follows from Fubini's theorem and the bi-invariance of the Haar measure on the unipotent subgroup $U$.

Since the compact group $M_{y, \vec{s}^{+}}=M_{y_{j+1}, \vec{s}^{+}}$normalizes $U_{y_{j+1}, \vec{s}}$ and acts on $v$ as a character $\chi_{\Gamma},($ iii) follows from (ii).

Proving $(i v)$, the second inclusion is obvious. To prove the first, since $v_{0} \in V_{\pi}^{\chi_{\Gamma}}$, we note that $U_{y_{0}, \vec{s}} \cup \bar{U}_{y_{0}, \vec{s}^{+}} \subset \operatorname{ker}\left(\chi_{\Gamma} \mid K_{y_{0} \vdash}\right)=\operatorname{Stab}\left(v_{0}\right)$. Since $U_{y_{1}, \vec{s}^{+}} \subset U_{y_{1}, \vec{s}}=$ $U_{y_{0}, \vec{s}}$ and $\bar{U}_{y_{1}, \vec{s}^{+}} \subset \bar{U}_{y_{0}, \vec{s}^{+}}$, the case $j=0$ follows. Now, suppose $j \geq 1$. We observe that $\bar{U}_{x_{j+1}, \vec{s}}=\bar{U}_{y_{j+1}, \vec{s}^{+}} \subset \bar{U}_{y_{j}, \vec{s}^{+}} \subset \operatorname{Stab}\left(v_{j-1}\right)$ by the induction hypothesis. Consider the character $\chi_{\Gamma}$ realized by $\Gamma$ on $K_{y_{j+1}}$. Denote the kernel of $\chi_{\Gamma}$ in $K_{y_{j+1} \vdash}$ by $A$. Then since $U_{y_{j+1}, \vec{s}}, \bar{U}_{y_{j+1}, \vec{s}^{+}} \subset A$ and $K_{y_{j+1} \vdash}$ is decomposable with respect to $P=M U, A$ is also decomposable with respect to $P=M U$. Let $A_{M}:=$ $A \cap M$. Then we have $A_{M} \subset \operatorname{Stab}\left(v_{j-1}\right)$ by (iii), and $A=U_{y_{j+1}, \vec{s}} A_{M} \bar{U}_{y_{j+1}, \vec{s}^{+}}=$ $\bar{U}_{y_{j+1}, \vec{s}^{+}} A_{M} U_{y_{j+1}, \vec{s}}$. Since $\bar{U}_{y_{j+1}, \vec{s}^{+}} A_{M}=A_{M} \bar{U}_{y_{j+1}, \vec{s}^{+}} \subset \operatorname{Stab}\left(v_{j-1}\right)$, for a proper 
normalization, we have

$$
\begin{aligned}
v_{j} & =\int_{U_{y_{j+1}, \vec{s}}} \pi(n) v_{j-1} d n \\
& =\int_{U_{y_{j+1}, \vec{s}}} \int_{A_{M}} \int_{\bar{U}_{y_{j+1}, \vec{s}^{+}}} \pi(n m \bar{n}) v_{j-1} d \bar{n} d m d n=\int_{A} \pi(a) d a .
\end{aligned}
$$

Hence $\bar{U}_{y_{j+1}, \vec{s}^{+}} \subset A \subset \operatorname{Stab}\left(v_{j}\right)$ and (iv) follows.

For $(v)$, if $j=0$, it is obvious from the choice of $v_{0}$. If $j \geq 1$, since $G_{x_{j}, 0^{+}}^{0}=$ $\bar{U}_{y_{j+1}, 0^{0}}^{0} M_{y, 0^{+}}^{0} U_{y_{j}, 0}^{0}$, and since $v_{j}$ is fixed by $U_{y_{j}, 0}^{0}$ and $\bar{U}_{y_{j+1}, 0}^{0}=\bar{U}_{y_{j+1}, 0^{+}}^{0}$, combining these with $(i i i),(v)$ follows.

The equalities in $(v i)$ result from the decomposition $U_{x_{j}, \vec{s}}=U_{x_{j}, 0}^{0} U_{x_{j}, s_{0}}^{1} \ldots$ $U_{x_{j}, s_{d-1}}^{d}$.

To prove $(i)$, let $w_{d+1}:=v_{j-1}$ and $w_{i}:=\int_{U_{x_{j}, s_{i-1}}^{i}} \pi\left(u_{i}\right) w_{i+1} d u_{i}$. Then $v_{j}=w_{0}$. As in the case of $(i i i)$ and $(i v)$, we have for $\ell \leq i, M_{y, s_{\ell-1}^{+}}^{\ell}$ acts on $w_{i}$ via $\chi_{\Gamma}$ and each $w_{i}$ is fixed by $\bar{U}_{x_{j}, s_{\ell-1}}^{\ell}$. By the induction hypothesis, $v_{j-1}=w_{d+1} \neq 0$. Suppose $w_{i+1} \neq 0$ with $i \neq 0$. Then consider the $\tilde{J}_{x_{j}}^{i}$ stable vector space $W_{i}$ generated by $\left\{\pi(g) w_{i+1} \mid g \in \tilde{J}_{x_{j}}^{i}\right\}$. Note that $w_{i+1}$ is fixed by $\bar{U}_{x_{j}, s_{i-1}}^{i}$ and $U_{x_{j}, s_{i-1}^{+}}^{i}$. Moreover, from $U_{x_{j}, s_{i-1}}^{i-1} \subset U_{x_{j}, s_{i-2}^{+}}^{i-1} \subset U_{x_{j}, s^{+}} \subset \operatorname{Stab}\left(v_{j-1}\right)$, we have that $U_{x_{j}, s_{i-1}}^{i-1}$ fixes $w_{i+1}$. Hence, the representation of $\tilde{J}_{x_{j}}^{i}$ on $W_{i}$ is a Heisenberg representation of $\tilde{J}_{x_{j}}^{i}$ twisted by a character $\chi_{\gamma}$ of $\tilde{J}_{x_{j}}^{i}$ represented by $\gamma=\Gamma_{i}+\Gamma_{i+1}+\cdots+\Gamma_{d}$ (recall that each $\Gamma_{i}, \Gamma_{i+1}, \cdots, \Gamma_{d}$ defines a character of $G^{i}$ ). Then by Corollary 16.5, $w_{i} \neq 0$. Inductively, $w_{1} \neq 0$. Since $w_{1}$ is fixed under $\bar{U}_{x_{j}, 0}^{0}$ and $U_{x_{j}, 0^{+}}^{0}$ and since $\bar{U}_{x_{j}, 0}^{0}$ and $U_{x_{j}, 0}^{0}$ project to the unipotent radicals of opposite parabolic subgroups in $G_{x_{j}, 0}^{0} / G_{x_{j}, 0^{+}}^{0}$, by Lemma 15.4, $w_{0}=v_{j}=\int_{U_{x_{j}, 0}^{0}} \pi\left(u_{0}\right) w_{1} d u_{0}$ is not zero.

Since any open compact subgroup of $U$ is contained in $U_{x_{j}, \vec{s}}$ for sufficiently large $j$, by $(i)$ and $(i i)$, we conclude that the image of $v$ under the Jacquet module map with respect to $U$ is nonzero. This is a contradiction to the supercuspidality of $\left(\pi, V_{\pi}\right)$. Hence, we conclude that $G_{y, 0}^{0}$ is maximal.

17.4. Proof of Proposition 17.2(2). Suppose $\tau$ is not cuspidal. Then there is $z \in$ $\mathcal{B}\left(\mathbf{G}^{0}, k\right)$ such that $G_{z, 0}^{0} \subsetneq G_{y, 0}^{0}$ and $\phi_{\Sigma}^{-1} \otimes V_{\pi}^{\chi_{\Gamma}}$ has $G_{z, 0^{+}}^{0}$ invariants. Let $\mathbf{T} \subset \mathbf{G}^{0}$ be an $E$-split maximal $k$-torus such that $\mathcal{A}\left(\mathbf{G}^{0}, \mathbf{T}, k\right)$ is of maximal dimension and $y, z \in \mathcal{A}\left(\mathbf{G}^{0}, \mathbf{T}, k\right)$. Let $\mathbf{M}$ be a $k$-Levi subgroup of $\mathbf{G}$ with $\mathbf{T} \subset \mathbf{M}$ adapted for $G_{z, 0}^{0}$. Let $\mathbf{C}$ be the center of $\mathbf{M}$. Fix a $k$-parabolic subgroup $\mathbf{P}$ with $\mathbf{P}=\mathbf{M U}$.

Since $\phi_{\Sigma}^{-1} \otimes V_{\pi}^{\chi_{\Gamma}}$ has $G_{z, 0^{+}}^{0}$ invariants, and since $M_{y, 0}^{0} U_{y, 0}^{0}$ projects to a parabolic subgroup in $G_{y, 0}^{0} / G_{y, 0^{+}}^{0}$ sharing the Levi factor with the image of $G_{z, 0}^{0}$, we can deduce from [16] (see also [23, (1.1)]) that $\phi_{\Sigma}^{-1} \otimes V_{\pi}^{\chi_{\Gamma}}$ also has $U_{y, 0}^{0}$ invariants. Then, by Corollary $13.12(2),\left(\pi, V_{\pi}\right)$ also contains $\left(K_{y \vdash}, \chi_{\Gamma}\right)$ where $K_{y \vdash}$ is defined with respect to $\mathbf{M U}$ as in 13.9 .

Let $\beta: \mathbf{G L}_{1} \rightarrow \mathbf{C}$ be a one-parameter subgroup of $\mathbf{C}$ such that $\langle\alpha, \beta\rangle>0$ for every root $\alpha$ of $\mathbf{C}$ in the Lie algebra $\mathfrak{u}$ of $U$. Take the ray $y(t)=y+t \beta, t \geq 0$, contained in the apartment $\mathcal{A}(\mathbf{G}, \mathbf{T}, k)$, emanating from the point $y$ in the direction of $\beta$. Now, the rest of the proof is similar to that of Proposition 17.2(1). 


\section{EXTENDING $\phi_{\Sigma}$ OF $K_{\Sigma}^{+}$TO $K^{d}$}

Let $\left(\pi, V_{\pi}\right)$ be a fixed irreducible supercuspidal representation of positive depth. Suppose $\Sigma=(\overrightarrow{\mathbf{G}}, y, \vec{r}, \vec{\phi})$ is a good $G$-datum of positive depth such that $\left(K_{\Sigma}^{+}, \phi_{\Sigma}\right)$ is contained in $\left(\pi, V_{\pi}\right)$. Define

$$
K:=\overrightarrow{\mathbf{G}}(k)_{\left(0^{+}, s_{0}, s_{1}, \cdots, s_{d-1}\right)}=G_{y, 0^{+}}^{0} G_{y, s_{0}}^{1} \cdots G_{y, s_{d-1}}^{d} .
$$

Note that $K^{d}=G_{[y]}^{0} K$ where $K^{d}$ is defined in 12.3 , To simplify notation, we denote $K_{\Sigma}^{+}$by $K^{+}$. Let $N:=\operatorname{ker}\left(\phi_{\Sigma}\right)$.

Proposition 18.1. $K / N$ is a Heisenberg p-group with center $K^{+} / N$.

To prove the above proposition, recall from $\$ 12.3$ that

$$
J_{+}^{i}=\left(\mathbf{G}^{i-1}, \mathbf{G}^{i}\right)(k)_{y,\left(r_{i-1}, s_{i-1}^{+}\right)}, \quad J^{i}=\left(\mathbf{G}^{i-1}, \mathbf{G}^{i}\right)(k)_{y,\left(r_{i-1}, s_{i-1}\right)} .
$$

Define a pairing on $J^{i} / J_{+}^{i}$ given by $\langle a, b\rangle_{i}=\phi_{\Sigma}\left(a b a^{-1} b^{-1}\right)$.

Note that $K=G_{y, 0^{+}}^{0} J^{1} \cdots J^{d}$ and $K^{+}=G_{y, 0^{+}}^{0} J_{+}^{1} \cdots J_{+}^{d}$.

\section{Lemma 18.2.}

(1) The pairing $\langle,\rangle_{i}$ is nondegenerate on $J^{i} / J_{+}^{i}$.

(2) $J^{i} /\left(N \cap J_{+}^{i}\right)$ is a Heisenberg p-group with center $J_{+}^{i} /\left(N \cap J_{+}^{i}\right)$.

Proof. Let $\hat{\phi}_{j}$ be as in $\$ 5.8$ or 12.4 . For $j>i-1$, since $a b a^{-1} b^{-1} \in\left(G^{j}, G^{j}\right)$, $\hat{\phi}_{j}\left(a b a^{-1} b^{-1}\right)=1$. For $j<i-1$, since $a b a^{-1} b^{-1} \in G_{y, r_{i-1}}^{i} \subset G_{y, r_{j}^{+}} \subset \operatorname{ker}\left(\hat{\phi}_{j}\right)$, $\hat{\phi}_{j}\left(a b a^{-1} b^{-1}\right)=1$. Hence $\langle a, b\rangle=\phi_{\Sigma}\left(a b a^{-1} b^{-1}\right)=\hat{\phi}_{j-1}\left(a b a^{-1} b^{-1}\right)$. Then this pairing coincides with the one in Lemma 11.1 of [38], and the above follows from that lemma.

Proof. Proof of Proposition 18.1 Define a pairing on $K / K^{+}$given by $\langle a, b\rangle=$ $\phi_{\Sigma}\left(a b a^{-1} b^{-1}\right)$. Note that $\langle$,$\rangle on J^{i} / J_{+}^{i} \subset K / K^{+}$coincides with $\langle,\rangle_{i}$. We claim that if $i \neq j,\langle$,$\rangle is trivial on J^{i} / J_{+}^{i} \times J^{j} / J_{+}^{j}$. Without loss of generality, we may assume $i<j$. Let $a \in J^{i}$ and $b \in J^{j}$. If $\ell<j$, since $\left(J^{i}, J^{j}\right) \subset J_{+}^{j} \subset \operatorname{ker}\left(\hat{\phi}_{\ell}\right)$, $\hat{\phi}_{\ell}\left(a b a^{-1} b^{-1}\right)=1$. If $\ell \geq j, a b a^{-1} b^{-1} \in\left(G^{j}, G^{j}\right) \subset\left(G^{\ell}, G^{\ell}\right)$ and $\hat{\phi}_{\ell}\left(a b a^{-1} b^{-1}\right)=1$.

Hence, we have $\langle\rangle=,\langle,\rangle_{1} \oplus \cdots \oplus\langle,\rangle_{d}$, and the pairing $\langle$,$\rangle is nondegenerate.$ Now, we can conclude $K / N$ is a Heisenberg $p$-group with center $K^{+} / N$.

18.3. By Proposition 18.1 and the theory of Heisenberg groups, there is a unique representation of $K$ extending the character $\phi_{\Sigma}$. Let $\phi_{\Sigma}^{\mathrm{h}}$ be the unique irreducible representation of $K$ extending the character $\phi_{\Sigma}$, and let $V_{\phi_{\Sigma}^{\mathrm{h}}} \subset V_{\pi}^{\phi_{\Sigma}}$ be the space of $\phi_{\Sigma}^{\mathrm{h}}$.

Let $\omega_{\pi}$ be the central character of $\left(\pi, V_{\pi}\right)$. Then, $Z_{G}$ acts via $\omega_{\pi}$ on $V_{\phi_{\Sigma}^{\mathrm{h}}}$. Let $\phi_{\Sigma}^{\mathrm{h} \pi}$ denote the irreducible representation of $Z_{G} K$ such that $\phi_{\Sigma}^{\mathrm{h} \pi}=\phi_{\Sigma}^{\mathrm{h}}$ on $K$ and $\phi_{\Sigma}^{\mathrm{h} \pi}=\omega_{\pi} \cdot 1$ on $Z_{G}$. Note that $\left(\pi, V_{\pi}\right)$ also contains $\left(Z_{G} K, \phi_{\Sigma}^{\mathrm{h} \pi}\right)$. Moreover, $G_{[y]}^{0}$ stabilizes the representation $\left(\phi_{\Sigma}^{\mathrm{h}}, V_{\phi_{\Sigma}^{\mathrm{h}}}\right)=\left(\phi_{\Sigma}^{\mathrm{h} \pi}, V_{\phi_{\Sigma}^{\mathrm{h}} \pi}\right)$. We fix an extension of $\phi_{\Sigma}^{\mathrm{h} \pi}$ of $Z_{G} K$ to $G_{[y]}^{0} K$ on $V_{\phi \frac{\mathrm{h}}{\Sigma}}$, which we denote by $\tilde{\phi}_{\Sigma}^{\mathrm{h} \pi}$.

Remark 18.4. Let $\kappa$ be as in $\$ 12.4$ associated to $\Sigma$. Then since $\kappa \mid K^{+}$is $\phi_{\Sigma}$-isotypic, $\kappa \mid K \simeq \phi_{\Sigma}^{\mathrm{h}}$. Hence we can choose the extension $\tilde{\phi}_{\Sigma}^{\mathrm{h} \pi}$ to be $\kappa$. 
Proposition 18.5. Any irreducible representation of $G_{[y]}^{0} K$ extending $\left(Z_{G} K, \phi_{\Sigma}^{\mathrm{h} \pi}\right)$ is of the form $\rho \otimes \tilde{\phi}_{\Sigma}^{\mathrm{h} \pi}$ where $\rho$ is an irreducible representation of $G_{[y]}^{0} K$ factoring through $Z_{G} K$.

Proof. Note that $\rho$ in the above induces an irreducible representation of $G_{[y]}^{0} / G_{y, 0^{+}}^{0}$. Then, since $\tilde{\phi}_{\Sigma}^{\mathrm{h} \pi} \mid K$ is already irreducible, the representation of the form $\rho \otimes \tilde{\phi}_{\Sigma}^{\mathrm{h} \pi}$ is also irreducible.

Let $\Theta_{1}$ (resp. $\left.\Theta_{2}\right)$ be the character of $\left(\operatorname{Ind}_{Z_{G} K}^{G_{\mid y]}^{0} K} 1\right) \otimes \tilde{\phi}_{\Sigma}^{\mathrm{h} \pi}\left(\right.$ resp. $\left.\operatorname{Ind}_{Z_{G} K}^{G_{[y]}^{0} K} \phi_{\Sigma}^{\mathrm{h} \pi}\right)$. Now, since $\left[G_{[y]}^{0}: G_{y, 0^{+}}^{0} Z_{G}\right]$ is finite, we can explicitly compute $\Theta_{1}$ and $\Theta_{2}$. Since $G_{[y]}^{0} K$ normalizes $Z_{G} K$, for $g, h \in G_{[y]}^{0} K,{ }^{h} g \in Z_{G} K$ if and only if $g \in Z_{G} K$. Hence,

$$
\Theta_{1}(g)=\Theta_{2}(g)= \begin{cases}\phi_{\Sigma}^{\mathrm{h} \pi}(g) & \text { if } g \in Z_{G} K \\ 0 & \text { otherwise }\end{cases}
$$

Therefore, $\left(\operatorname{Ind}_{Z_{G} K}^{G_{[y]}^{0} K} 1\right) \otimes \tilde{\phi}_{\Sigma}^{\mathrm{h} \pi}$ and $\operatorname{Ind}_{Z_{G} K}^{G_{[y]}^{0} K} \phi_{\Sigma}^{\mathrm{h} \pi}$ are isomorphic, and the proposition follows from this.

Corollary 18.6. We keep the notation from Proposition 18.5. Let $\left(\pi, V_{\pi}\right)$ be as before. Then there is a strongly good $G$-datum of positive depth $\Sigma=(\overrightarrow{\mathbf{G}}, y, \vec{r}, \vec{\phi})$ and an irreducible representation $\rho$ of $G_{[y]}^{0} K$ factoring through $Z_{G} K$ such that $\left(\pi, V_{\pi}\right)$ contains $\left(G_{[y]}^{0} K, \rho \otimes \kappa\right)$, where $\kappa$ is as in $§ 12.4$. Moreover, $c-\operatorname{Ind}_{G_{[y]}^{0}}^{G^{0}} \rho$ is an irreducible supercuspidal representation of $G^{0}$

Proof. The first statement follows from Corollary 11.5, \$18.3, Remark 18.4, and Proposition 18.5. The second follows from Proposition [17.2 and [31, (6.6)].

\section{Conclusion: Exhaustion of Supercuspidal Representations}

Theorem 19.1. Suppose $(\mathrm{H} k),(\mathrm{HB}),(\mathrm{HGT})$ and $(\mathrm{H \mathcal {N }})$ are valid. Then any irreducible supercuspidal representation $\left(\pi, V_{\pi}\right)$ of $G$ arises through Yu's construction.

Proof. Let $\left(\pi, V_{\pi}\right)$ be a given irreducible supercuspidal representation. If the depth $\varrho(\pi)$ of $\left(\pi, V_{\pi}\right)$ is zero, this follows from Remark 12.6. Hence, we may assume $\varrho(\pi)>$ 0 . Then by Corollary 18.6, there is a strongly good $G$-datum of positive depth $\Sigma=(\overrightarrow{\mathbf{G}}, y, \vec{r}, \vec{\phi})$ and an irreducible representation $\rho$ of $G_{[y]}^{0} K$ factoring through $Z_{G} K$ such that $\left(\pi, V_{\pi}\right)$ contains $\left(G_{[y]}^{0} K, \rho \otimes \kappa\right)$. Moreover, $\mathbf{Z}_{\mathbf{G}^{0}} / \mathbf{Z}_{\mathbf{G}}$ is $k$-anisotropic by Proposition 14.5, and $c$ - $\operatorname{Ind}_{G_{[y]}^{0}}^{G^{0}} \rho$ is an irreducible supercuspidal representation of $G^{0}$ from Corollary 18.6. Hence, $\Sigma_{Y}=(\vec{G}, y, \vec{r}, \vec{\phi}, \rho)$ is a generic $G$-datum. Let $\pi_{\Sigma_{Y}}:=c-\operatorname{Ind}_{G_{[y]}^{0} K}^{G}(\rho \otimes \kappa)$, the supercuspidal representation constructed in 38. Since $\rho \otimes \kappa<\pi$, from the Frobenius reciprocity and the irreducibility of $\left(\pi, V_{\pi}\right)$, we conclude $\pi \simeq \pi_{\Sigma_{Y}}$.

Corollary 19.2. Suppose $(\mathrm{H} k)$, (HB), (HGT) and (HN) are valid. Then, all supercuspidal representations of $G$ are compactly induced from an irreducible representation of an open compact mod center group. 


\section{SELECTED NOTATion}

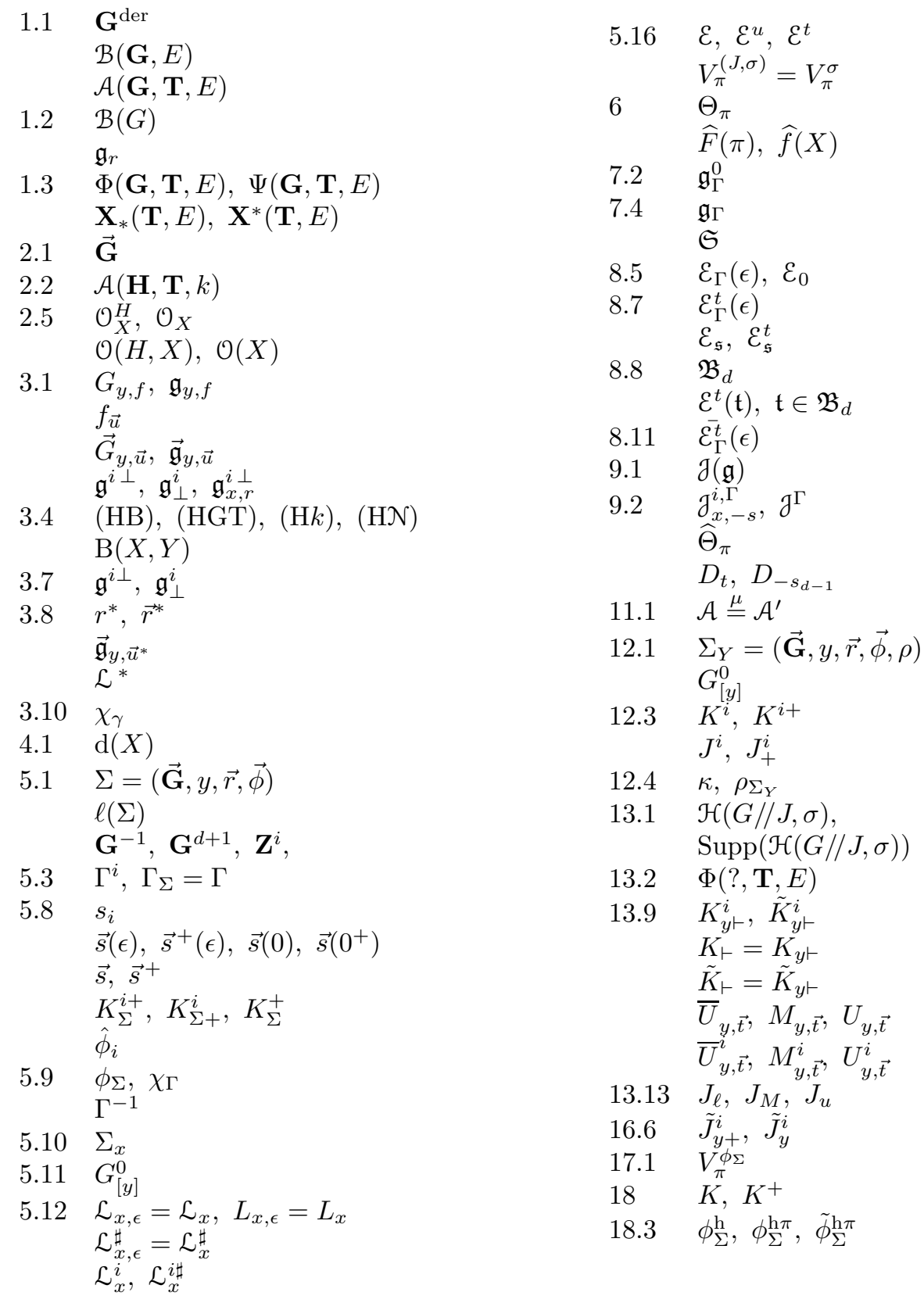

\section{ACKNOWLEDGMENTS}

I thank Fiona Murnaghan for the joint work on character expansions, which has been an important tool for this work. Part of this paper was motivated by a conversation with Roger Howe, whom I thank for the inspiration. I would like to thank Joseph Bernstein, Allen Moy, Fiona Murnaghan and Jiu-Kang Yu for many enlightening discussions and Anne-Marie Aubert, Stephen DeBacker, Jonathan Korman 
and Loren Spice for their helpful comments on the earlier version of this paper. Finally, I would like to thank Tom Hales and Paul Sally for their interest and warm support of this work.

\section{ADDED IN PROOF}

This paper was originally submitted for publication in 2004. Very recently (July 2006), a preprint appeared (Shaun Stevens, The supercuspidal representations of $p$-adic classical groups, math.RT/0607622) whose results have a substantial overlap with the ones here. Stevens' approach is based on the strategy of Bushnell and Kutzko [5], which is completely different from the one here. It is limited to classical groups but allows for more general residue characteristics (any odd residue characteristic $p$ is allowed). For small $p$, tame supercuspidal representations are not enough, and Stevens actually constructs some new supercuspidal representations which complement the tame ones.

\section{REFERENCES}

[1] J. Adler, Refined anisotropic K-types and supercuspidal representations, Pacific J. Math. 185 (1998), 1-32. MR1653184 (2000f:22019)

[2] J. Adler and S. DeBacker, Some applications of Bruhat-Tits theory to harmonic analysis on the Lie algebra of a reductive p-adic group, Michigan J. Math. 50 (2002), no. 2, 263-286. MR.1914065 (2003g:22016)

[3] _ Murnaghan-Kirillov theory for supercuspidal representations of tame general linear groups, J. Reine Angew. Math. 575 (2004), 1-35. MR2097545 (2005j:22008)

[4] J. Adler and A. Roche, An intertwining result for p-adic groups, Canad. J. Math. 52 (2000), no. 3, 449-467. MR.1758228 (2001m:22032)

[5] C. Bushnell and P. Kutzko, The admissible dual of $G L(N)$ via compact open subgroups, 129, Annals of Math Studeis, Princeton Univ. Press, 1993. MR.1204652 (94h:22007)

[6] , The admissible dual of $S L(N)$ I, 26, Ann. Sci. ENS, 261-280. MR1209709 (94a:22033)

[7] , The admissible dual of $S L(N)$ II, Proc. London Math. Soc. 68 (1994), 317-379. MR.1253507 (94k:22035)

[8] Smooth representations of reductive p-adic groups: structure theory via types, Proc. London Math. Soc. 77 (1998), no. 3, 582-634. MR.1643417 (2000c:22014)

[9] F. Bruhat and J. Tits, Groupes réductifs sur un corps local I, Publ. Math. I.H.E.S. 41 (1972), 5-251. MR 0327923 (48:6265)

[10] W. Casselman, Introduction to the theory of admissible representation of p-adic reductive groups, preprint.

[11] S. DeBacker, Homogeneity results for invariant distributions of a reductive p-adic group, Ann. Sci. École Norm. Sup. (4) 35 (2002), 391-422. MR1914003(2003i:22019)

[12] _ Parameterizing nilpotent orbits via Bruhat-Tits theory, Ann. of Math. 156 (2002), 295-331. MR1935848 (2003i:20086)

[13] J. Dixmier, Les $C^{*}$-algèbres et leurs représentations, Éditions Jacques Gabay, Paris, 1996. MR.1452364 (98a:46066)

[14] J. Hakim and F. Murnaghan, Distinguished tame supercuspidal representations, work in progress.

[15] Harish-Chandra, Admissible invariant distributions on reductive p-adic groups, Preface and notes by Stephen DeBacker and Paul J. Sally, Jr., University Lecture Series, 16, American Mathematical Society, Providence, RI, 1999. MR1702257 (2001b:22015)

[16] _ Eisenstein series over finite fields, Collected papers, Springer Verlag, New York, 1984. MR0457579 (56:15784)

[17] — The Plancherel formula for reductive $p$-adic groups, Collected Papers, 4, SpringerVerlag, Berlin, 1976 MR 0726026 (85e:01061d)

[18] R. Howe, The Fourier transform and germs of characters (case of $G l_{n}$ over a p-adic field), Math. Ann. 208 (1974), 305-322. MR0342645 (49:7391) 
[19] Some qualitative results on the representation theory of $G l_{n}$ over a p-adic field, Pacific J. of Math. 73 (1977), 479-538. MR0492088 (58:11242)

[20] - Tamely ramified supercuspidal representations of $G l_{n}$, Pacific J. of Math. 73 (1977), 437-460. MR0492087 (58:11241)

[21] R. Howe with collaboration of A. Moy, Harish Chandra Homomorphisms for p-adic groups, vol. 59, CBMS regional conference series, AMS, Providence, RI, 1985. MR0821216 (87h:22023)

[22] R. Howe and A. Moy, Hecke algebra isomorphisms for $G L(n)$ over a p-adic field, J. Algebra 131 (1990), 388-424. MR.1058553 (91f:22031)

[23] R. Howlett and G. Lehrer, On Harish-Chandra induction and restriction for modules of Levi subgroups, J. Algebra 165 (1994), 172-183. MR1272585 (95d:20025)

[24] J. L. Kim, Hecke algebras of classical groups over p-adic fields and supercuspidal representations, Amer. J. Math. 121 (1999), 967-1029. MR.1713299 (2001g:11075)

[25] _ Dual blobs and Plancherel formulas, Bull. Soc. Math. France 132 (2004), no. 1, 55-80. MR2075916 (2005f:22025)

[26] J. L. Kim and F. Murnaghan, Character expansions and unrefined minimal K-types, Amer. J. Math. 125 (2003), 1199-1234. MR2018660 (2004k:22024)

[27] , K-types and $\Gamma$-asymptotic expansions, J. Reine Angew. Math. 592 (2006), 189-236. MR.2222734

[28] L. Morris, Level zero G-types, Compositio Math. 118 (1999), 135-157. MR1713308 (2000g:22029)

[29] A. Moy, Local constants and the tame Langlands correspondence, Amer. J. Math. 108 (1986), 863-930. MR0853218 (88b:11081)

[30] A. Moy and G. Prasad, Unrefined minimal K-types for p-adic groups, Inv. Math. 116 (1994), 393-408. MR1253198 (95f:22023)

[31] — Jacquet functors and unrefined minimal K-types, Comment. Math. Helvetici 71 (1996), 98-121. MR1371680(97c:22021)

[32] G. Prasad, Galois fixed points in the Bruhat-Tits buildings of a reductive group, Bulletin Soc. Math. France 129 (2001), no. 2, 169-174. MR.1871292 (2002j:20088)

[33] G. Rousseau, Immeubles des groupes réductifs sur les corps locaux, Thèse, Paris XI (1977). MR 0491992 (58:11158)

[34] S. Stevens, Semisimple characters for p-adic classical groups, Duke Math. J. 127 (2005), no. 1, 123-173. MR2126498 (2006a:22017)

[35] M. Tadic, Geometry of dual spaces of reductive groups, J. d'Analyse Math. 51 (1988), 139181. MR0963153 (90c:22057)

[36] J.-L. Waldspurger, Homogénéité de certaines distributions sur les groupes p-adiques, Inst. Hautes Études Sci. Publ. Math. No. 81 (1995), 25-72. MR1361755 (98f:22023)

[37] — La formule de Plancherel pour les groupes p-adiques d'après Harish-Chandra, J. Inst. Math. Jussieu 2 (2003), no. 2, 235-333. MR1989693 (2004d:22009)

[38] J. K. Yu, Construction of tame supercuspidal representations, J. Amer. Math. Soc. 14 (2001), no. 3, 579-622. MR:1824988 (2002f:22033)

Department of Mathematics, University of Illinois at Chicago, Chicago, Illinois 60607

E-mail address: julee@math.uic.edu

Current address: Department of Mathematics, Massachusetts Institute of Technology, 2-275,

Cambridge, Massachusetts 02139

E-mail address: julee@math.mit.edu 